\title{
Stabilization of U(III) to Oxidation and Hydrolysis by Encapsulation
}

\author{
Using 2.2.2-Cryptand \\ Supplementary Information \\ Daniel N. Huh ${ }^{\dagger}$, Jeffrey M. Barlow ${ }^{\dagger}$, Sierra R. Ciccone, Joseph W. Ziller, \\ Jenny Y. Yang*, and William J. Evans* \\ Department of Chemistry, University of California, Irvine, California 92697, United States \\ Email: wevans@uci.edu, j.yang@uci.edu \\ ${ }^{\dagger}$ Authors contributed equally to this work.
}




\section{Table of Contents}

Experimental details on the synthesis and electrochemistry of 1-U, 3-U, 4-U,

5-U, and 6-U.

Figure S1. UV-Vis of $[\mathrm{U}(\mathrm{crypt})(\mathrm{MeCN})(\mathrm{I})][\mathrm{I}]_{2}, \mathbf{4 - U}$

S8

Electrochemical Data

Figure S2. Cyclic voltammograms comparing $3 \mathrm{mM}$ [U(crypt) $\left.\mathrm{I}_{2}\right] \mathrm{I}, \mathbf{1}-\mathbf{U}, 9 \mathrm{mM}$

tetra- $n$-butylammonium iodide (TBAI), and electrolyte only.

Figure S3. Cyclic voltammograms comparing $3 \mathrm{mM}$ solutions of [U(crypt) $\left.\mathrm{I}_{2}\right] \mathrm{I}$ 1-U,

S9

$\mathrm{UI}_{3}$, empty cryptand, and [K(crypt)[OTf] in DMF.

Figure S4. Variable scan rate voltammograms of the first oxidation event (E $\left.\mathrm{E}_{\mathrm{PA} 1}\right)$ of

1-U in DMF from 10 to $500 \mathrm{mV} / \mathrm{s}$ scan rates.

Figure S5. Additional variable scan rate voltammograms of the first oxidation event $\left(E_{P A 1}\right)$ of $\mathbf{1 - U}$ in DMF from 10 to $5000 \mathrm{mV} / \mathrm{s}$ scan rates.

Figure S6. Current vs. square root of scan rate plot for the first oxidation of 1-U in $\mathrm{DMF}\left(\mathrm{E}_{\mathrm{PA} 1}\right)$ and its return reduction wave $\left(\mathrm{E}_{\mathrm{PC} 1}\right)$.

Figure S7. Variable scan rate voltammograms of the second oxidation event (EA2) of 1-U in DMF from 10 to $500 \mathrm{mV} / \mathrm{s}$ scan rates.

Figure S8. Current vs. square root of scan rate plot for the second oxidation of 1-U in DMF (E $\left.\mathrm{E}_{\mathrm{PA} 2}\right)$ between 50 to $1000 \mathrm{mV} / \mathrm{s}$ scan rate.

Table S1. Recorded anodic $\left(i_{a}\right)$ and cathodic $\left(i_{c}\right)$ currents, current ratios $\left(i_{a} / i_{c}\right)$, and peak-to-peak separation $(\Delta \mathrm{E})$ for the proposed $\mathrm{U}(\mathrm{IV} / \mathrm{III})$ couple of 1-U at varying scan rates in DMF.

Figure S9. Variable scan rate voltammograms of 1-U in DMF scanning anodically between 10 to $2500 \mathrm{mV} / \mathrm{s}$ scan rates.

Figure S10. Variable scan rate voltammograms of the first oxidation event $\left(\mathrm{E}_{\mathrm{PA} 1}\right)$ of [U(crypt) $\left.\mathrm{I}_{2}\right] \mathrm{I}$ in $\mathrm{MeCN}$ from 100 to $5000 \mathrm{mV} / \mathrm{s}$.

Figure S11. Current vs. square root of scan rate plot for the first oxidation of [U(crypt)I $\left.\mathrm{I}_{2}\right] \mathrm{I}$ in $\mathrm{MeCN}$ (EPA1) between 100 to $5000 \mathrm{mV} / \mathrm{s}$ scan rate.

Figure S12. Variable scan rate voltammograms of the first reduction $\left(\mathrm{E}_{\mathrm{PC} 1}\right)$ and oxidation $\left(\mathrm{E}_{\mathrm{PA} 2}\right)$ events of $\left[\mathrm{U}(\mathrm{crypt}) \mathrm{I}_{2}\right] \mathrm{I}$ observed in $\mathrm{MeCN}$ when scanning cathodically from 10 to $1000 \mathrm{mV} / \mathrm{s}$. 
Figure S13. Current vs. square root of scan rate plot for the first oxidation (EPA2) and first reduction $\left(\mathrm{E}_{\mathrm{PC} 1}\right)$ of $\left[\mathrm{U}(\mathrm{crypt}) \mathrm{I}_{2}\right] \mathrm{I}$ observed when scanning cathodically in $\mathrm{MeCN}$ between 10 to $1000 \mathrm{mV} / \mathrm{s}$ scan rate.

Figure $S 14$. Variable scan rate voltammograms of [U(crypt) $\left.I_{2}\right]$ I scanning cathodically $S 15$ in $\mathrm{MeCN}$ from 10 to $1000 \mathrm{mV} / \mathrm{s}$ showing all oxidation and reduction events.

Figure $\mathrm{S} 15$. Cyclic voltammograms comparing $3 \mathrm{mM}$ solutions of [U(crypt) $\left.\mathrm{I}_{2}\right] \mathrm{I}$ (1-U), $\mathrm{UI}_{3}$, empty cryptand, and [K(crypt)[OTf] in MeCN.

Figure S16. Individual cyclic voltammograms of 1-U in DMF with increasing concentrations of added water.

Figure S17. Cyclic voltammograms of $\mathbf{1 - U}$ in DMF with increasing concentrations of water in the presence or absence of $3 \mathrm{mM} \mathbf{1 - U}$ in $0.2 \mathrm{M} \mathrm{TBAPF}_{6} \mathrm{DMF}_{\text {. }}$

Figure S18. Cyclic voltammograms of $\mathbf{1 - U}$ in $\mathrm{MeCN}$ with increasing concentrations of added water lacking redox events arising from free iodide in solution. Voltammograms are recorded scanning cathodically from OCP.

Figure S19. Cyclic voltammograms of $\mathbf{1 - U}$ in $\mathrm{MeCN}$ with increasing concentrations of added water lacking redox events arising from free iodide in solution. Voltammograms are recorded scanning anodically from OCP.

Figure S20. Cyclic voltammograms of $\mathbf{1 - U}$ in $\mathrm{MeCN}$ with increasing concentrations of added water. Voltammograms are recorded scanning anodically. from OCP.

Figure S21. Cyclic voltammograms of $\mathbf{1 - U}$ in $\mathrm{MeCN}$ with increasing concentrations of added water. Voltammograms are recorded scanning cathodically.

Figure S22. Electronic absorption spectra of [U(crypt) $\left.\mathrm{I}_{2}\right] \mathrm{I}$ in $\mathrm{MeCN}$ after SEC $\mathrm{UV}-\mathrm{Vis}$ at $-2.0 \mathrm{~V}\left(\mathrm{vs} . \mathrm{Fe}\left(\mathrm{C}_{5} \mathrm{H}_{5}\right)_{2}{ }^{+/ 0}\right)$ applied potential.

Figure S23. Electronic absorption spectra of [U(crypt) $\left.\mathrm{I}_{2}\right] \mathrm{I}$ in $\mathrm{MeCN}$ after SEC $\mathrm{UV}-\mathrm{Vis}$ at $-0.15 \mathrm{~V}\left(\mathrm{vs} . \mathrm{Fe}\left(\mathrm{C}_{5} \mathrm{H}_{5}\right)_{2}{ }^{+/ 0}\right)$ applied potential.

Figure S24. Electronic absorption spectra in $\mathrm{MeCN}$ of $0.20 \mathrm{mM}$ [U(crypt) $\left.\mathrm{I}_{2}\right] \mathrm{I}$ and $0.3 \mathrm{mM}$ isolated oxidation product after reaction with $1 \mathrm{eq}$ $\left[\mathrm{Fe}\left(\mathrm{C}_{5} \mathrm{H}_{5}\right)_{2}\right]\left[\mathrm{BF}_{4}\right]$.

Table S2. Reported U(IV/III) reduction potentials $\left[\mathrm{E}_{1 / 2}\left(\mathrm{U}^{\mathrm{IV} / \mathrm{III}}\right)\right]$ for various 
uranium complexes including solvent and reference utilized for the measurement.

Figure S25. Cyclic Voltammogram of $\left[\mathrm{Co}\left(\mathrm{C}_{5} \mathrm{H}_{5}\right)_{2}\right]\left[\mathrm{PF}_{6}\right]$ under $\mathrm{N}_{2}$ atmosphere in DMF.

Figure S26. Cyclic Voltammograms in MeCN comparing 1-U using $\mathrm{TBAPF}_{6}$ electrolyte and 1-U using $\mathrm{TBABPh}_{4}$ electrolyte. Voltammograms are recorded scanning anodically.

Figure S27. Cylcic Voltammograms in $\mathrm{MeCN}$ comparing 1-U using $\mathrm{TBAPF}_{6}$ electrolyte and 1-U using $\mathrm{TBABPh}_{4}$ electrolyte. Voltammograms are recorded scanning cathodically.

Crystallographic Data

$[\mathrm{U}($ crypt $)(\mathrm{MeCN}) \mathrm{I}][\mathrm{I}]_{2}, \mathbf{4 - U}$

Table S3. Crystal data and structure refinement for 4-U. S24

Table S4. Selected bond lengths $[\AA]$ and angles $\left[{ }^{\circ}\right]$ for $\mathbf{4 - U} . \quad$ S25

$\left[\mathrm{U}(\right.$ crypt $\left.)\left(\mathrm{OH}_{2}\right)_{2}\right][\mathrm{I}]_{3} \cdot 2 \mathrm{MeCN}, \mathbf{5}-\mathbf{U} \quad$ S26

Table S5. Crystal data and structure refinement for 5-U. S26

Table S6. $\quad$ Selected bond lengths $[\AA]$ and angles $\left[{ }^{\circ}\right]$ for 5-U. $\quad$ S27

$\begin{array}{llr}\text { Table S7. Hydrogen bonds for 5-U, }\left[\AA \text { and }{ }^{\circ}\right] . & \text { S29 }\end{array}$

$\begin{array}{lll}\text { Figure S28. Hydrogen bonding between two units of 5-U. S29 } & \text { S.U. }\end{array}$

$\left[\mathrm{U}(\mathrm{crypt})(\mathrm{DMF})\left(\mathrm{OH}_{2}\right)\right][\mathrm{I}]_{3} \cdot \mathrm{H}_{2} \mathrm{O}, \mathbf{6}-\mathbf{U} \quad \mathrm{S} 29$

Table S8. Crystal data and structure refinement 6-U. S30

Table S9. Selected bond lengths $[\AA]$ and angles $\left[{ }^{\circ}\right]$ for $\mathbf{6}-\mathbf{U} . \quad$ S31

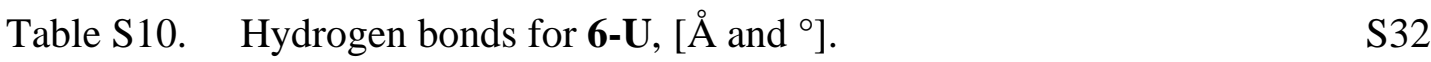

Figure S29. Hydrogen bonding between two units of 6-U. S32

$\left[\mathrm{H}_{2}\right.$ (crypt) $][\mathrm{I}]_{2} \quad$ S33

$\begin{array}{lll}\text { Figure } \mathrm{S} 30 . & \text { Solid state structure of }\left[\mathrm{H}_{2}(\mathrm{crypt})\right][\mathrm{I}]_{2} . & \text { S33 }\end{array}$

Table S11. Crystal data and structure refinement for $\left[\mathrm{H}_{2}\right.$ (crypt) $][\mathrm{I}]_{2} . \quad \mathrm{S} 34$

Table S12. Bond lengths $[\AA]$ and angles $\left[{ }^{\circ}\right]$ for $\left[\mathrm{H}_{2}(\right.$ crypt $\left.)\right][\mathrm{I}]_{2} . \quad$ S35

Table S13. Hydrogen bonds for $\left[\mathrm{H}_{2}\right.$ (crypt) $][\mathrm{I}]_{2}\left[\AA\right.$ and $\left.^{\circ}\right] . \quad \mathrm{S} 35$

$\begin{array}{ll}\text { References } & \text { S36 }\end{array}$ 


\section{Experimental}

All syntheses and manipulations described below were conducted under argon or nitrogen with exclusion of air using glovebox, Schlenk-line, and high-vacuum techniques. [U(crypt)I $\left.\mathrm{I}_{2}\right]$, 1U, was prepared using previously published procedures. ${ }^{1} \quad$ 2.2.2-Cryptand $(4,7,13,16,21,24-$ hexaoxa-1,10-diazabicyclo[8.8.8] hexacosane, Aldrich) was placed under vacuum for $12 \mathrm{~h}\left(1 \times 10^{-}\right.$ ${ }^{3}$ Torr) before use. Electrochemical grade tetrabutylammonium hexafluorophosphate $(>99.0 \%$, Aldrich) was dried at $110{ }^{\circ} \mathrm{C}$ under vacuum for $12 \mathrm{~h}\left(1 \times 10^{-3}\right.$ Torr $)$ before use. Decamethyl ferrocene $\left[\mathrm{Fe}\left(\mathrm{C}_{5} \mathrm{Me}_{5}\right)_{2}\right]$ was purified by sublimation before use. Cobaltocenium hexafluorophosphate $\left(\left[\mathrm{Co}\left(\mathrm{C}_{5} \mathrm{H}_{5}\right)_{2}\right]\left[\mathrm{PF}_{6}\right]\right)(99 \%$, Strem) was used without further purification. THF, MeCN, DMF, and $\mathrm{Et}_{2} \mathrm{O}$ were sparged with UHP argon and dried over columns containing neutral alumina or Q-5 and molecular sieves, and then were stored over $4 \AA$ molecular sieves for at least one week. ${ }^{1} \mathrm{H}$ NMR spectra were obtained on a CRYO500 MHz spectrometer at $298 \mathrm{~K}$. Elemental analyses were conducted on a PerkinElmer 2400 Series II CHNS elemental analyzer. Infrared spectra were collected on an Agilent Cary 630 equipped with a diamond ATR attachment.

[U(crypt)I I I, 1-U. In an argon-filled glovebox, a blue solution of $\mathrm{UI}_{3}(100 \mathrm{mg}, 0.160$ $\mathrm{mmol})$ in THF (2 mL) was added dropwise to a stirred colorless THF (2 mL) solution of 2.2.2cryptand (60 mg, $0.160 \mathrm{mmol})$. A green/brown precipitate immediately formed. This mixture was stirred for $30 \mathrm{~min}$. The solvent was removed in vacuo yielding a light green solid (123 mg, $76 \%)$. The solid was dissolved in $\mathrm{CH}_{2} \mathrm{Cl}_{2}(2 \mathrm{~mL})$. The green solution was filtered and then layered with $\mathrm{Et}_{2} \mathrm{O}$ and placed in $\mathrm{a}-35^{\circ} \mathrm{C}$ freezer. After $1 \mathrm{~d}$, X-ray quality green crystals were isolated. IR: 3243w, 3169w, 2943w, 2926w, 2894w, 2860w, 1611w, 1490w, 1479m, 1468m, 1451m, $1437 \mathrm{w}, 1361 \mathrm{w}, 1354 \mathrm{w}, 1335 \mathrm{w}, 1311 \mathrm{w}, 1288 \mathrm{w}, 1262 \mathrm{~m}, 1240 \mathrm{w}, 1159 \mathrm{w}, 1103 \mathrm{~s}, 1085 \mathrm{~s}, 1075 \mathrm{~s}$, 
1050m, 1029m, 960s, 949m, 911w, 873w, 832m, 823m, 803w, 763w, 757w cm ${ }^{-1}$. Anal. Calcd. for [U(crypt) $\left.\mathrm{I}_{2}\right] \mathrm{I}, \mathrm{C}_{18} \mathrm{H}_{36} \mathrm{~N}_{2} \mathrm{O}_{6} \mathrm{I}_{3} \mathrm{U}: \mathrm{C}, 21.72 ; \mathrm{H}, 3.65 ; \mathrm{N}, 2.81$. Found: C, 21.92; H, 3.49; N, 2.52 .

$\left[\mathbf{U}(\mathbf{c r y p t})\left(\mathbf{O H}_{\mathbf{2}}\right) \mathbf{I}\right][\mathbf{I}]_{2}, \mathbf{3 - U} . \mathbf{M e C N}(3 \mathrm{~mL})$ was added to green $\left[\mathrm{U}(\right.$ crypt $\left.) \mathbf{I}_{2}\right] \mathrm{I}(100 \mathrm{mg}, 0.10$ mmol) to form a green-yellow solution. The solution was stirred for $1 \mathrm{~h}$, layered with $\mathrm{Et}_{2} \mathrm{O}$ and placed in a $-35^{\circ} \mathrm{C}$ freezer. After $1 \mathrm{~d}, \mathrm{X}$-ray quality green crystals were isolated and it was found to have the same unit cell as the previously reported compound. ${ }^{1}$

$[\mathbf{U}(\mathbf{c r y p t})(\mathbf{M e C N}) \mathbf{I}][\mathbf{I}]_{2}, 4-\mathbf{U} . \mathrm{MeCN}$ that was dried over $4 \AA$ molecular sieves for 1 week was transferred to a new set of $4 \AA$ molecular sieves and dried for an additional week. This rigorously dried $\mathrm{MeCN}(3 \mathrm{~mL})$ was added to green $\left[\mathrm{U}\left(\right.\right.$ crypt) $\left.\mathrm{I}_{2}\right] \mathrm{I}(71.2 \mathrm{mg}, 0.07 \mathrm{mmol})$, which formed a green-yellow solution. The solution was stirred for $1 \mathrm{~h}$, layered with $\mathrm{Et}_{2} \mathrm{O}$, and placed in a $-35^{\circ} \mathrm{C}$ freezer. After $1 \mathrm{~d}$, X-ray quality green crystals were isolated $(66 \mathrm{mg}, 90 \%) .{ }^{1} \mathrm{H}$ NMR $\left(\mathrm{CD}_{3} \mathrm{CN}\right.$ ): $\delta 5.99$ (br s, $\mathrm{OCH}_{2} \mathrm{CH}_{2} \mathrm{~N}, 12 \mathrm{H}$ ), 6.93 (br s, $\mathrm{OCH}_{2} \mathrm{CH}_{2} \mathrm{~N}, 12 \mathrm{H}$ ), 8.38 (br s, $\mathrm{OCH}_{2} \mathrm{CH}_{2} \mathrm{O}$, 12H). IR ( $\left.\mathrm{cm}^{-1}\right)$ : 2889m, 2297w, 2263w, 1447m, 1356m, 1257m, 1070s, 948s, 813m, 751m. Anal. Calcd for $\mathrm{C}_{20} \mathrm{H}_{39} \mathrm{I}_{3} \mathrm{~N}_{3} \mathrm{O}_{6} \mathrm{U}: \mathrm{C}, 23.18 ; \mathrm{H}, 3.79 ; \mathrm{N}, 4.05$. Found: C, 23.25; H, 3.93; N, 3.75. The UV-vis spectrum is in Figure S1 below.

$\left[\mathbf{U}(\mathbf{c r y p t})\left(\mathbf{O H}_{2}\right)_{2}\right][]_{3} \cdot \mathbf{2 M e C N}, \mathbf{5 - U}$. As described for 3-U, MeCN was added to green [U(crypt) $\left.\mathrm{I}_{2}\right] \mathrm{I}(100 \mathrm{mg}, 0.10 \mathrm{mmol})$ forming a green-yellow solution. The solution was stirred for $1 \mathrm{~h}$, layered with $\mathrm{Et}_{2} \mathrm{O}$ and placed in a $-35^{\circ} \mathrm{C}$ freezer. After $1 \mathrm{~d}, \mathrm{X}$-ray quality green crystals were isolated.

[U(crypt)(DMF)(OH $)][\mathbf{I}]_{3}, \mathbf{6}-\mathbf{U}$. As described for 3-U, DMF (3 mL) was added to green [U(crypt) $\left.\mathrm{I}_{2}\right] \mathrm{I}(100 \mathrm{mg}, 0.10 \mathrm{mmol})$ forming a green-yellow solution. The solution was stirred for $1 \mathrm{~h}$, layered with $\mathrm{Et}_{2} \mathrm{O}$ and placed in a $-35^{\circ} \mathrm{C}$ freezer. After $1 \mathrm{~d}, \mathrm{X}$-ray quality green crystals were isolated. 
Physical Methods: Electronic absorption spectra were recorded using a $1 \mathrm{~cm}$ quartz cuvette with an Agilent Cary 60 UV-Vis spectrophotometer fitted with an Agilent fiber optic coupler connected to an Ocean Optics CUV $1 \mathrm{~cm}$ cuvette holder in a glovebox under an inert atmosphere of $\mathrm{N}_{2}$. UVVisible spectroelectrochemistry (SEC UV-Vis) was performed using a commercially available UV-Vis spectroelectrochemistry kit from Pine Instruments with a Pt working/counter electrode and $\mathrm{Ag}$ wire as a pseudo reference electrode. $\left[\mathrm{Co}\left(\mathrm{C}_{5} \mathrm{H}_{5}\right)_{2}\right]\left[\mathrm{PF}_{6}\right]$ was used as an internal standard.

Electrochemistry: All measurements were performed on a Pine Wavedriver 10 bipotentiostat with a $2 \mathrm{~mm}$ diameter glassy carbon disc working electrode, glassy carbon rod counter electrode, and a $\mathrm{Ag}^{+} / \mathrm{Ag}$ pseudoreference electrode separated from the bulk solution by a Vicor frit. Internal resistance was measured for each solution, and resistance manually compensated for between $80-$ $90 \%$ of the measured value for each voltammogram performed. Potentials are referenced to ferrocene $\left(\mathrm{Fe}\left(\mathrm{C}_{5} \mathrm{H}_{5}\right)_{2}{ }^{+/ 0}\right)$ at $0.00 \mathrm{~V}$ using either 1 equivalent $\left[\mathrm{Co}\left(\mathrm{C}_{5} \mathrm{H}_{5}\right)_{2}\right]\left[\mathrm{PF}_{6}\right]\left(\mathrm{E}_{1 / 2}=-1.33 \mathrm{~V}\right.$ vs. $\mathrm{Fe}\left(\mathrm{C}_{5} \mathrm{H}_{5}\right)_{2}{ }^{+/ 0}$, Figure S25) or excess $\mathrm{Fe}\left(\mathrm{C}_{5}\left(\mathrm{CH}_{3}\right)_{5}\right)_{2}\left(\mathrm{E}_{1 / 2}=-0.48 \mathrm{~V} \text { vs. } \mathrm{Fe}\left(\mathrm{C}_{5} \mathrm{H}_{5}\right)_{2}{ }^{+/ 0}\right)^{2}$ as an internal standard. All experiments were performed in dried and degassed dimethylformamide or acetonitrile solutions with $3.0 \mathrm{mM}$ analyte and $0.2 \mathrm{M}$ tetrabutylammonium hexafluorophosphate ( $\mathrm{TBAPF}_{6}$ ) supporting electrolyte concentrations and were recorded at a $100 \mathrm{mV} / \mathrm{s}$ scan rate unless otherwise noted.

Spectroelectrochemical UV-Vis Spectrophotometry (SEC UV-Vis): $0.40 \mathrm{~mL}$ of $2.50 \mathrm{mM}$ $\left[\mathrm{U}(\right.$ crypt $\left.) \mathrm{I}_{2}\right] \mathrm{I}$ in $0.2 \mathrm{M} \mathrm{TBAPF}_{6} \mathrm{MeCN}$ was placed into a Pine Instruments UV-Visible spectroelectrochemistry setup. An initial electronic absorption spectrum was collected before a cyclic voltammogram was measured. The solution was then electrolyzed about $200 \mathrm{mV}$ past (cathodic for reduction, and anodic for oxidation reactions) the peak potential of the desired redox event while electronic absorption spectra were collected every 5-10 seconds until the spectrum 
was observed to no longer change between scans. Spectra of reduced $\left[\mathrm{U}(\mathrm{crypt}) \mathrm{I}_{2}\right] \mathrm{I}$ were recorded for several minutes after current was no longer being passed to observe potential decomposition of formed species.

Water Stability Studies: Water stability studies were performed using degassed Milli-Q purified water (18 $\Omega . c m$ resistivity) in DMF or MeCN solutions containing $3 \mathrm{mM}\left[\mathrm{U}\right.$ (crypt) $\left.\mathrm{I}_{2}\right] \mathrm{I}$ and $0.2 \mathrm{M}$ $\mathrm{TBAPF}_{6}$. Solutions were mixed for five minutes upon water addition, after which open circuit potential and uncompensated resistance were measured and cyclic voltammograms performed.

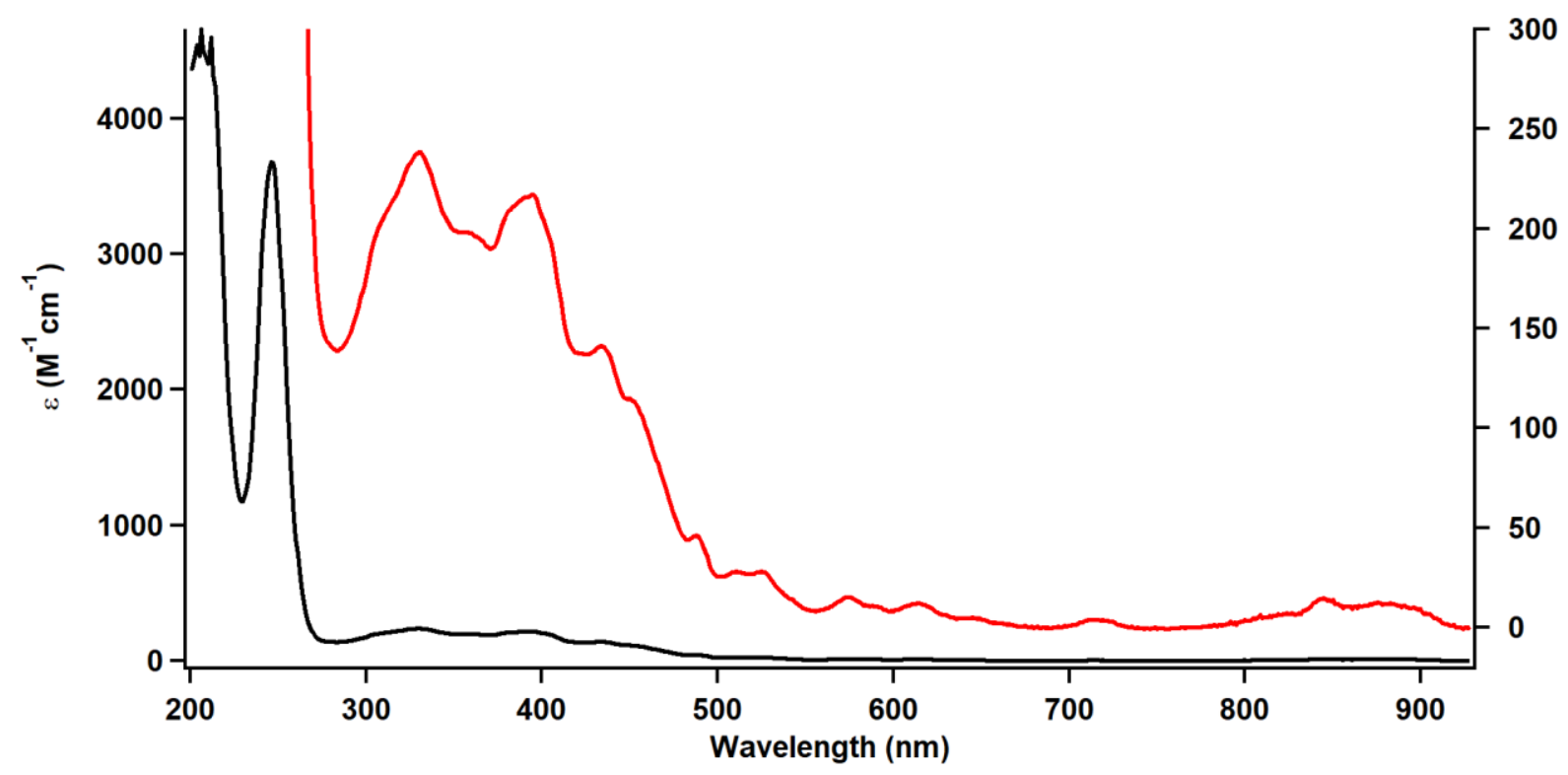

Figure S1. UV-Vis of $8 \mathrm{mM}$ [U(crypt)(MeCN)I]I, 4-U, in MeCN. Full spectrum (black, left axis) and zoom of full spectrum (red, right axis). 


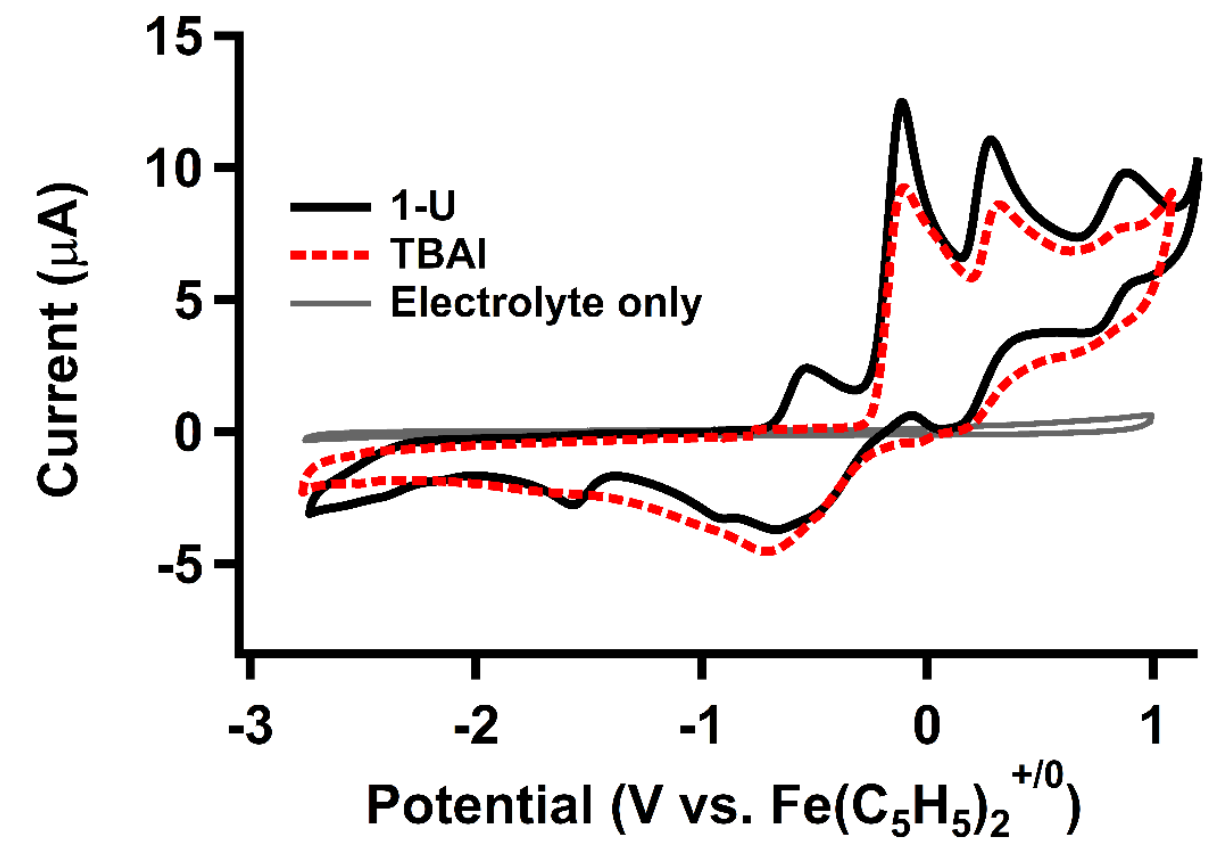

Figure S2. Cyclic voltammograms comparing $3 \mathrm{mM}$ [U(crypt) $\left.\mathrm{I}_{2}\right] \mathrm{I}, \mathbf{1 - U}$ (solid black trace), $9 \mathrm{mM}$ tetra- $n$-butylammonium iodide (TBAI, red dotted trace), and electrolyte only (solid grey trace). Voltammograms were recorded in $0.2 \mathrm{M} \mathrm{TBAPF}_{6} \mathrm{DMF}$ solutions scanning anodically at $100 \mathrm{mV} / \mathrm{s}$ scan rate.

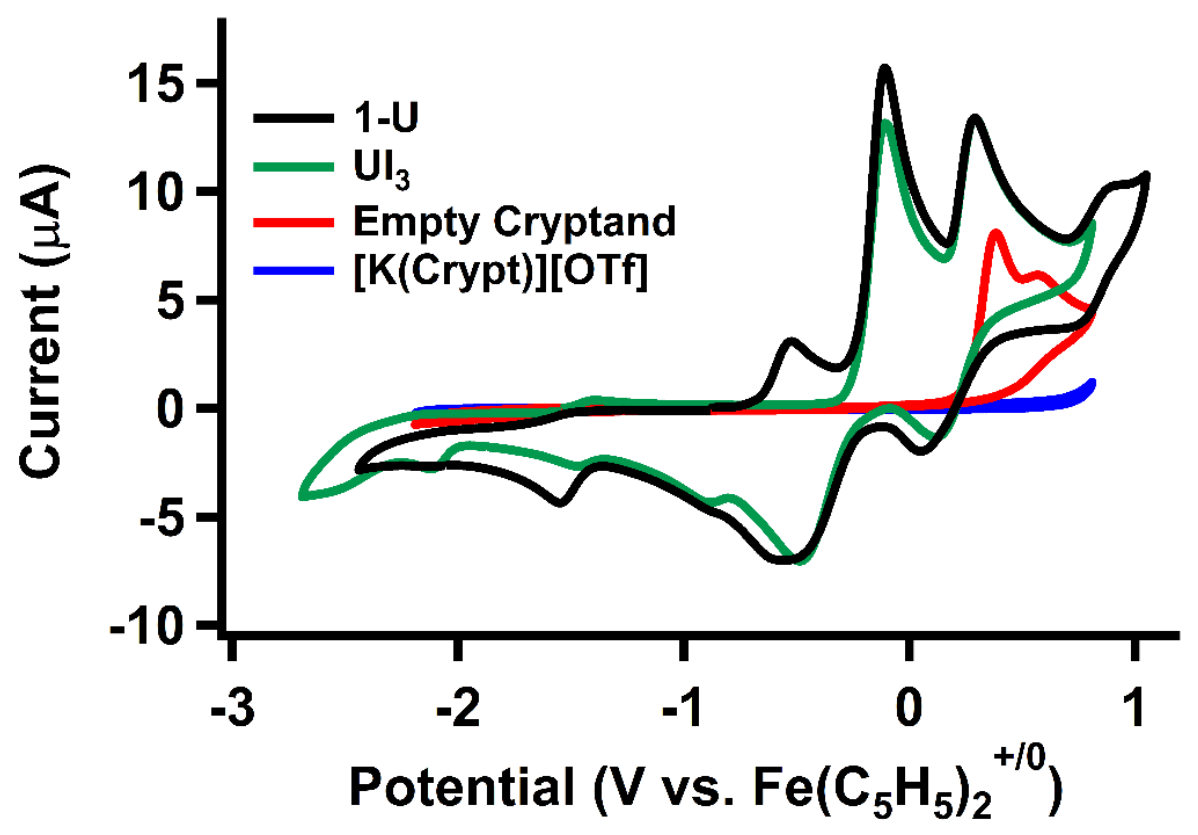

Figure S3. Cyclic voltammograms comparing $3 \mathrm{mM}$ solutions of [U(crypt) $\left.\mathrm{I}_{2}\right] \mathrm{I}$, 1-U (solid black trace), $\mathrm{UI}_{3}$ (solid green trace), empty cryptand (solid red trace), and [K(crypt)[OTf] (solid blue trace). Voltammograms were recorded in $0.2 \mathrm{M} \mathrm{TBAPF}_{6} \mathrm{DMF}$ solutions scanning anodically at $100 \mathrm{mV} / \mathrm{s}$ scan rate. 


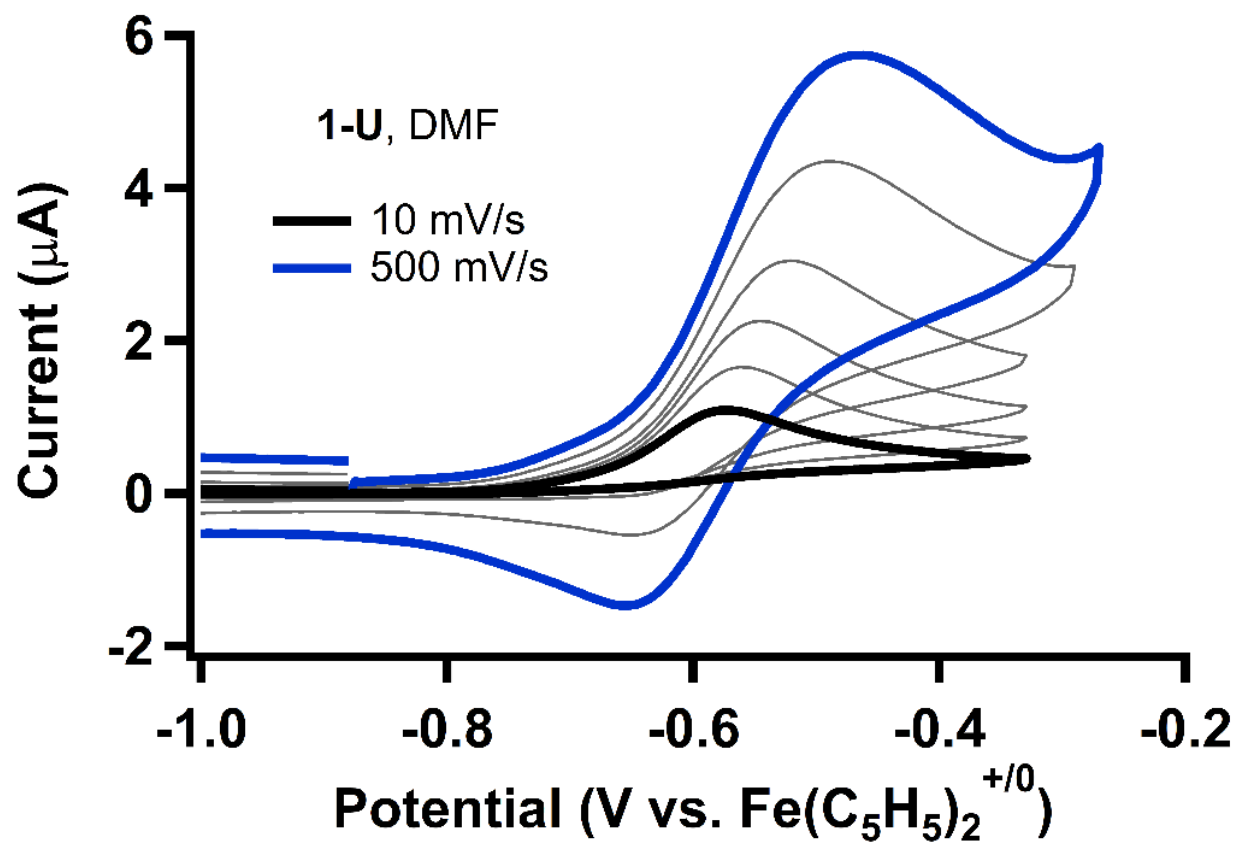

Figure S4. Variable scan rate voltammograms of the first oxidation event (EPA $)$ of 1-U in DMF from 10 to $500 \mathrm{mV} / \mathrm{s}$ scan rates, showing an increase in return wave current as the scan rate is increased.

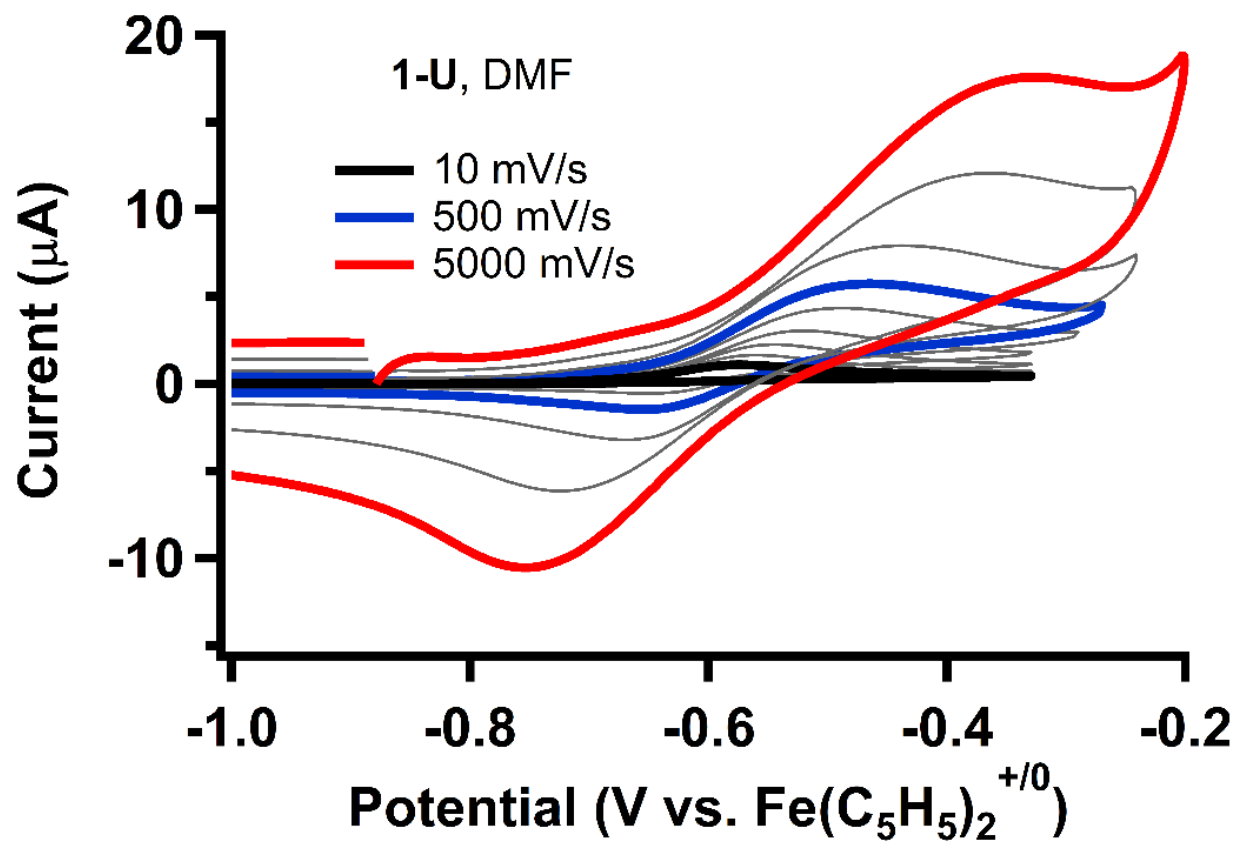

Figure S5. Additional variable scan rate voltammograms of the first oxidation event (EPA1) of 1$\mathbf{U}$ in DMF from 10 to $5000 \mathrm{mV} / \mathrm{s}$ scan rates, showing that the return wave continues to grow in as the scan rate is increased. 


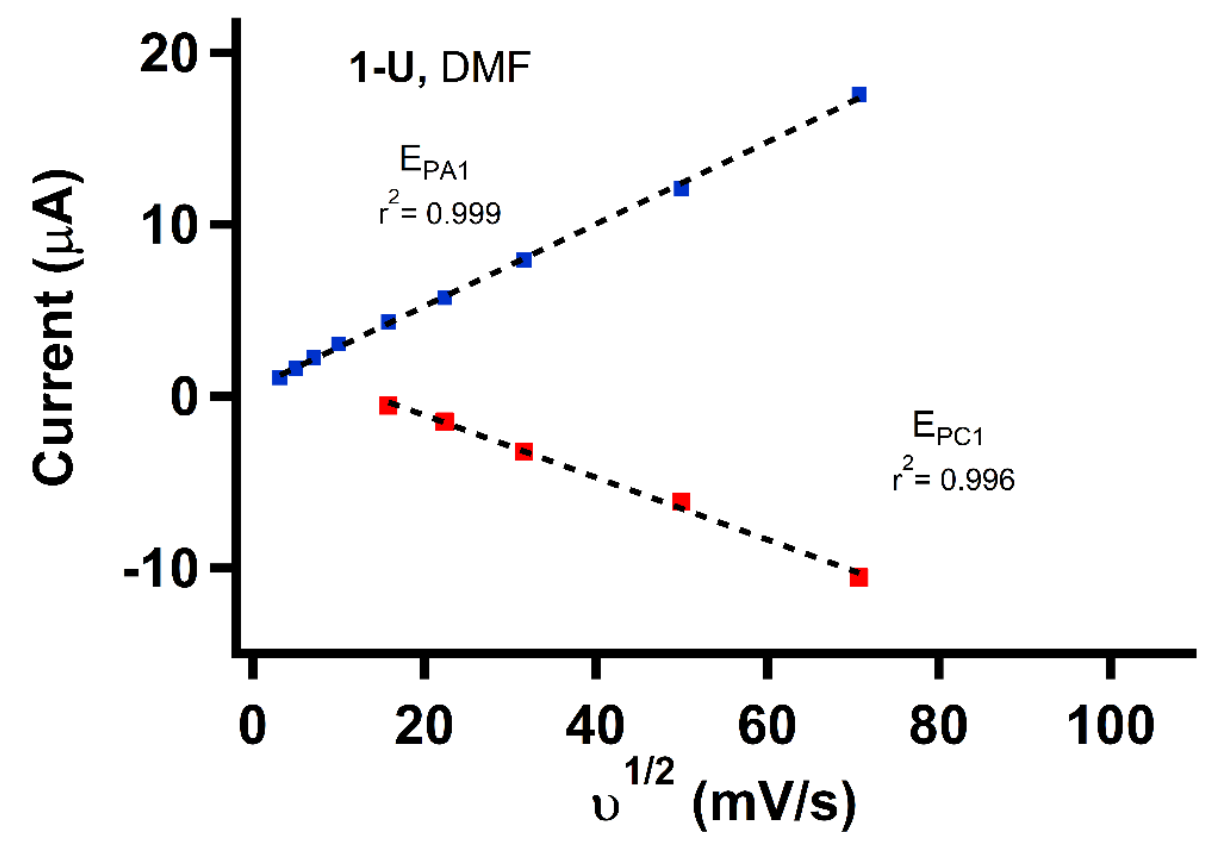

Figure S6. Current vs. square root of scan rate plot for the first oxidation of 1-U in DMF (EPA1, blue) and its return reduction wave $\left(\mathrm{E}_{\mathrm{PC} 1}\right.$, red) that grows in at faster scan rates.

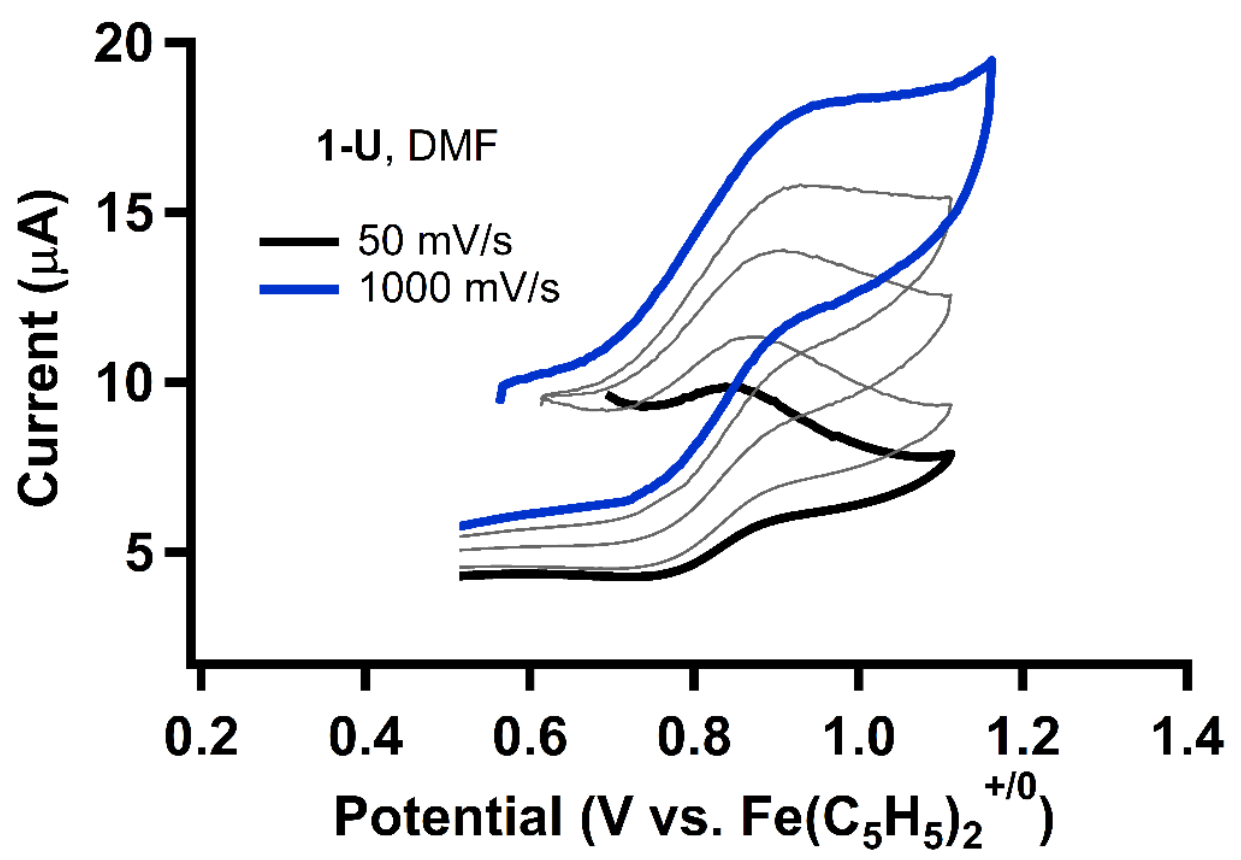

Figure S7. Variable scan rate voltammograms of the second oxidation event ( $\left.\mathrm{E}_{\mathrm{PA} 2}\right)$ of 1-U in DMF from 10 to $500 \mathrm{mV} / \mathrm{s}$ scan rates, showing an increase in return wave current as the scan rate is increased. 


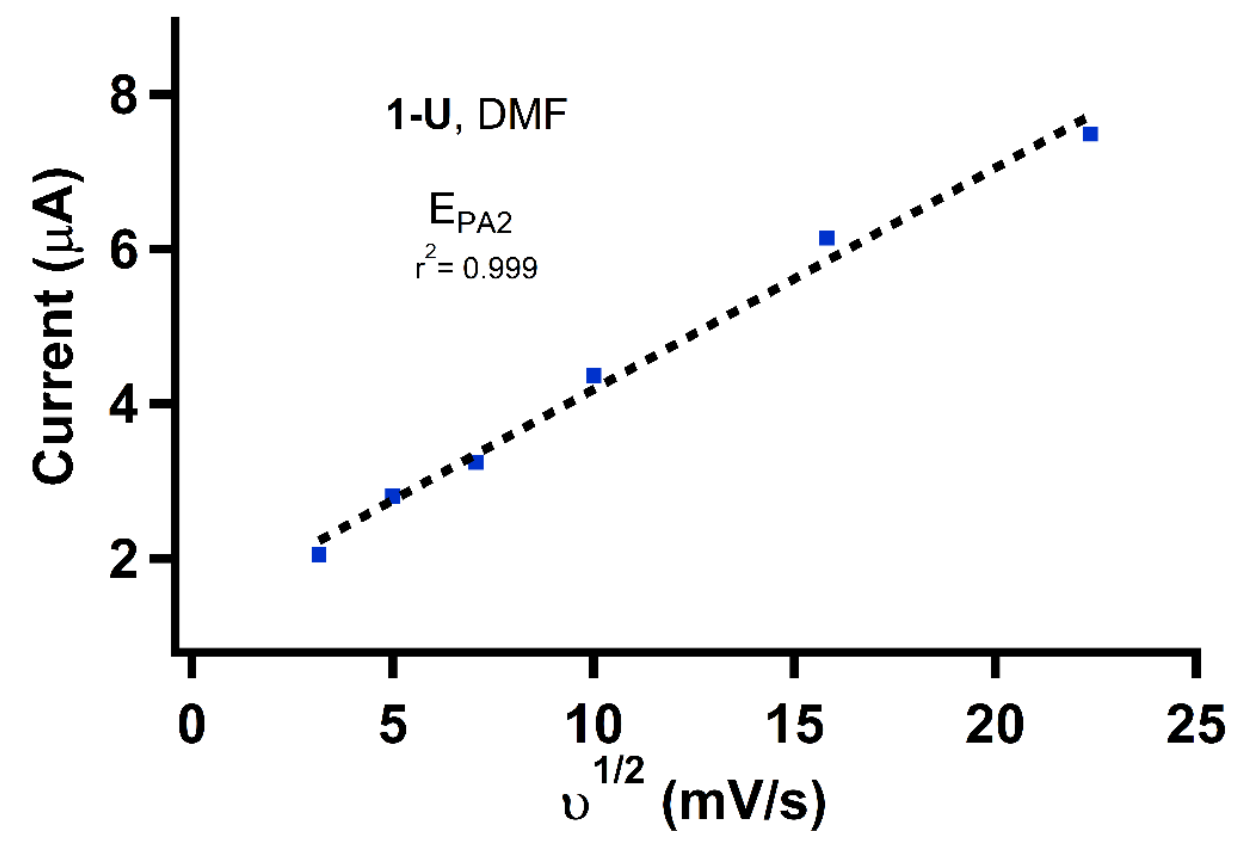

Figure S8. Current vs. square root of scan rate plot for the second oxidation of 1-U in DMF (E $\left.\mathrm{E}_{\mathrm{PA} 2}\right)$ between 50 to $1000 \mathrm{mV} / \mathrm{s}$ scan rate.

Table S1. Recorded anodic $\left(i_{a}\right)$ and cathodic $\left(i_{c}\right)$ currents, current ratios $\left(i_{a} / i_{c}\right)$, and peak-to-peak separation $(\Delta \mathrm{E})$ for the proposed $\mathrm{U}(\mathrm{IV} / \mathrm{III})$ couple of $\mathbf{1 - U}$ at varying scan rates in DMF.

\begin{tabular}{|c|c|c|c|c|}
\hline $\begin{array}{c}\text { Scan rate } \\
(\mathbf{m V} / \mathbf{s})\end{array}$ & $\mathbf{i}_{\mathbf{a}}(\boldsymbol{\mu A})$ & $\mathbf{i}_{\mathbf{c}}(\boldsymbol{\mu} \mathbf{A})$ & $\mathbf{i}_{\mathbf{a}} / \mathbf{i}_{\mathbf{c}}$ & $\boldsymbol{\Delta} \mathbf{E}(\mathbf{m V})$ \\
\hline $\mathbf{1 0}$ & 1.093 & - & - & - \\
\hline $\mathbf{2 5}$ & 1.654 & - & - & - \\
\hline $\mathbf{5 0}$ & 2.256 & - & - & - \\
\hline $\mathbf{1 0 0}$ & 3.048 & - & - & - \\
\hline $\mathbf{2 5 0}$ & 4.348 & -0.5439 & 7.994 & 150 \\
\hline $\mathbf{5 0 0}$ & 5.741 & -1.469 & 3.907 & 190 \\
\hline $\mathbf{1 0 0 0}$ & 7.933 & -3.210 & 2.471 & 230 \\
\hline $\mathbf{2 5 0 0}$ & 12.091 & -6.143 & 1.968 & 350 \\
\hline $\mathbf{5 0 0 0}$ & 17.589 & -10.541 & 1.6686 & 420 \\
\hline
\end{tabular}




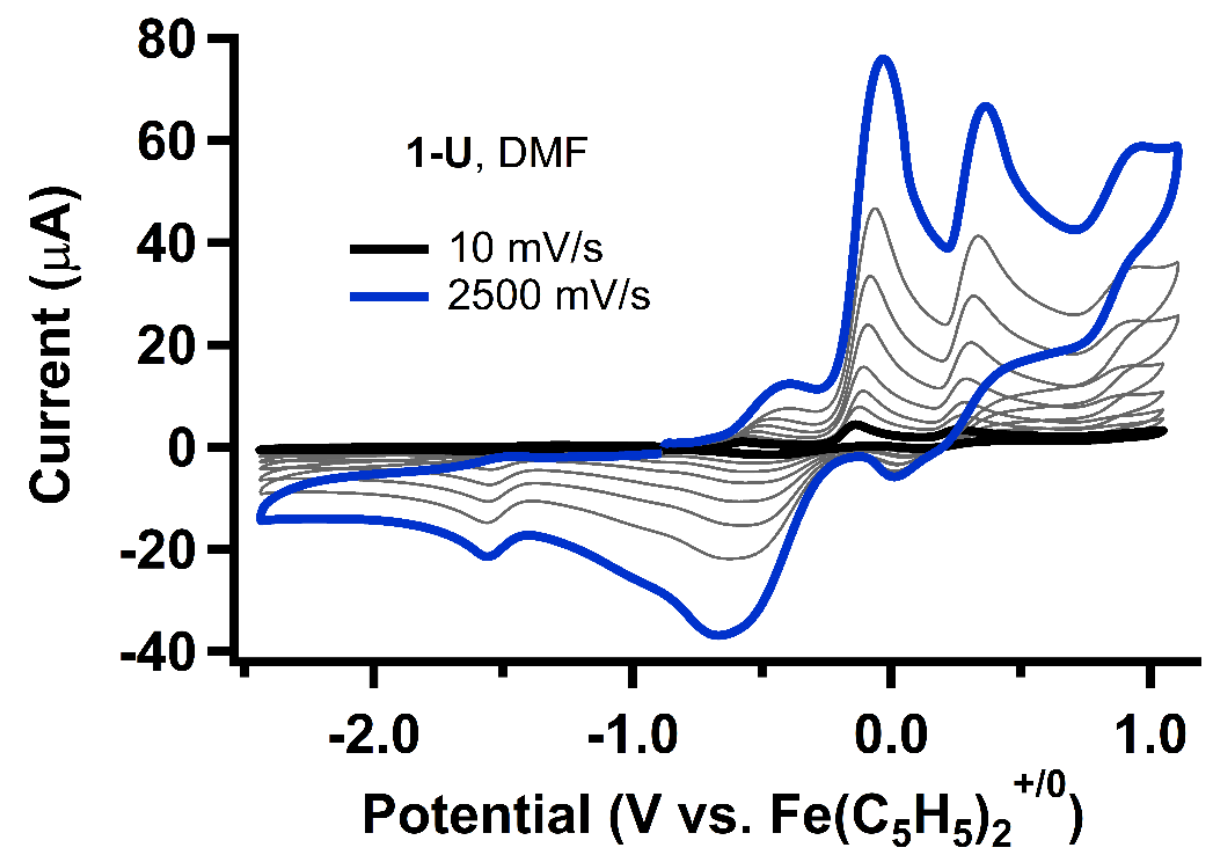

Figure S9. Variable scan rate voltammograms of 1-U in DMF scanning anodically between 10 to $2500 \mathrm{mV} / \mathrm{s}$ scan rates, showing all oxidation and reduction events. Voltammograms were recorded using $3 \mathrm{mM}$ analyte in a DMF solution containing $0.2 \mathrm{M} \mathrm{TBAPF}_{6}$.

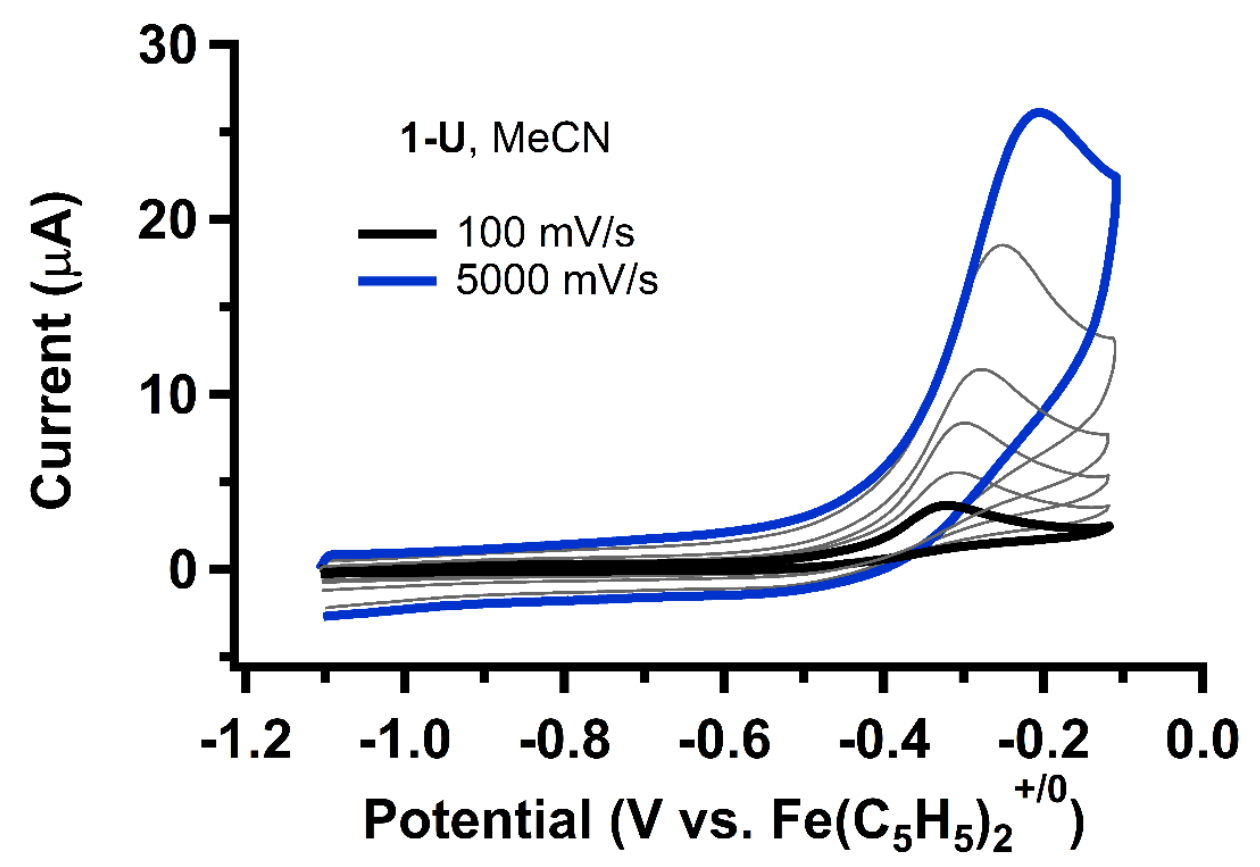

Figure S10. Variable scan rate voltammograms of the first oxidation event (EPA1) of [U(crypt) $\left.\mathrm{I}_{2}\right] \mathrm{I}$ in $\mathrm{MeCN}$ from 100 to $5000 \mathrm{mV} / \mathrm{s}$. 


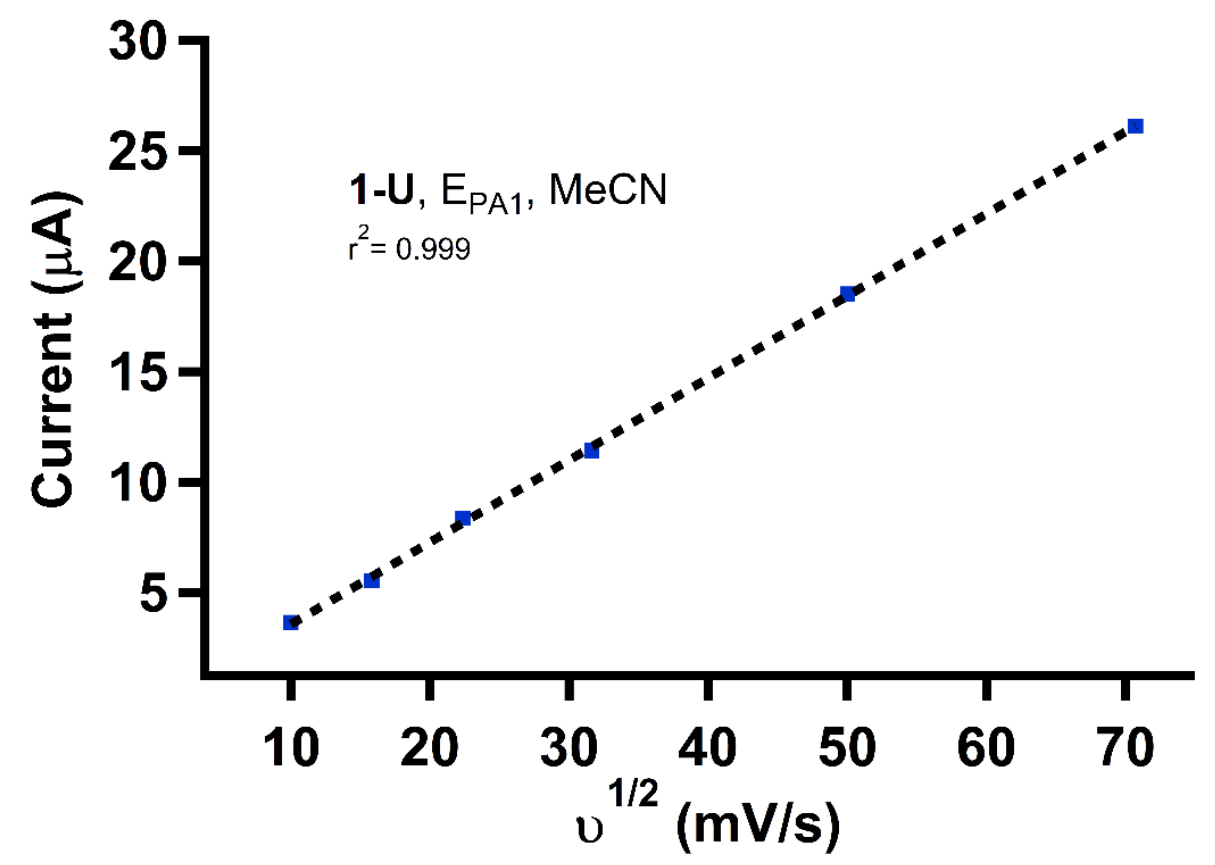

Figure S11. Current vs. square root of scan rate plot for the first oxidation of [U(crypt) $\left.\mathrm{I}_{2}\right] \mathrm{I}$ in $\mathrm{MeCN}\left(\mathrm{E}_{\mathrm{PAl}}\right.$ ) between 100 to $5000 \mathrm{mV} / \mathrm{s}$ scan rate.

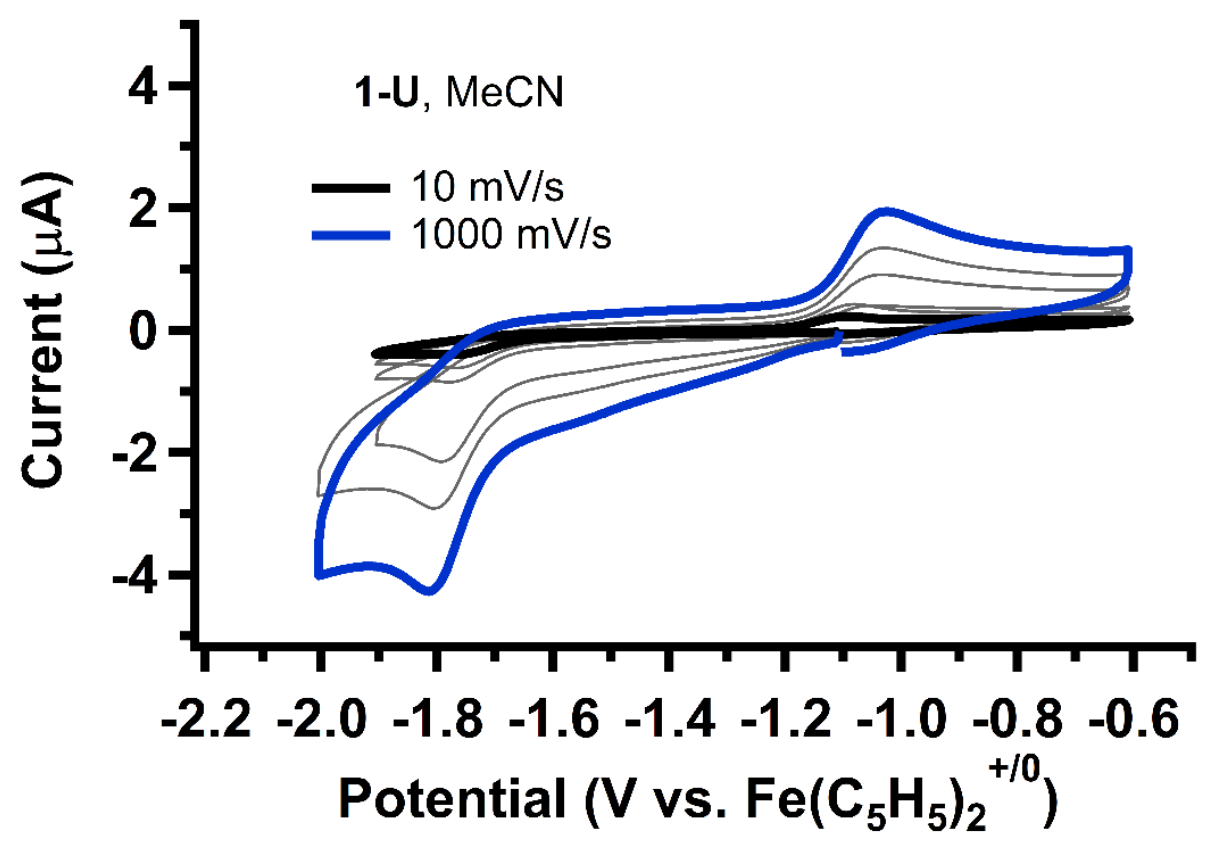

Figure S12. Variable scan rate voltammograms of the first reduction $\left(\mathrm{E}_{\mathrm{PC} 1}\right)$ and oxidation $\left(\mathrm{E}_{\mathrm{PA} 2}\right)$ events of [U(crypt) $\left.\mathrm{I}_{2}\right] \mathrm{I}$ observed in $\mathrm{MeCN}$ when scanning cathodically from 10 to $1000 \mathrm{mV} / \mathrm{s}$. 


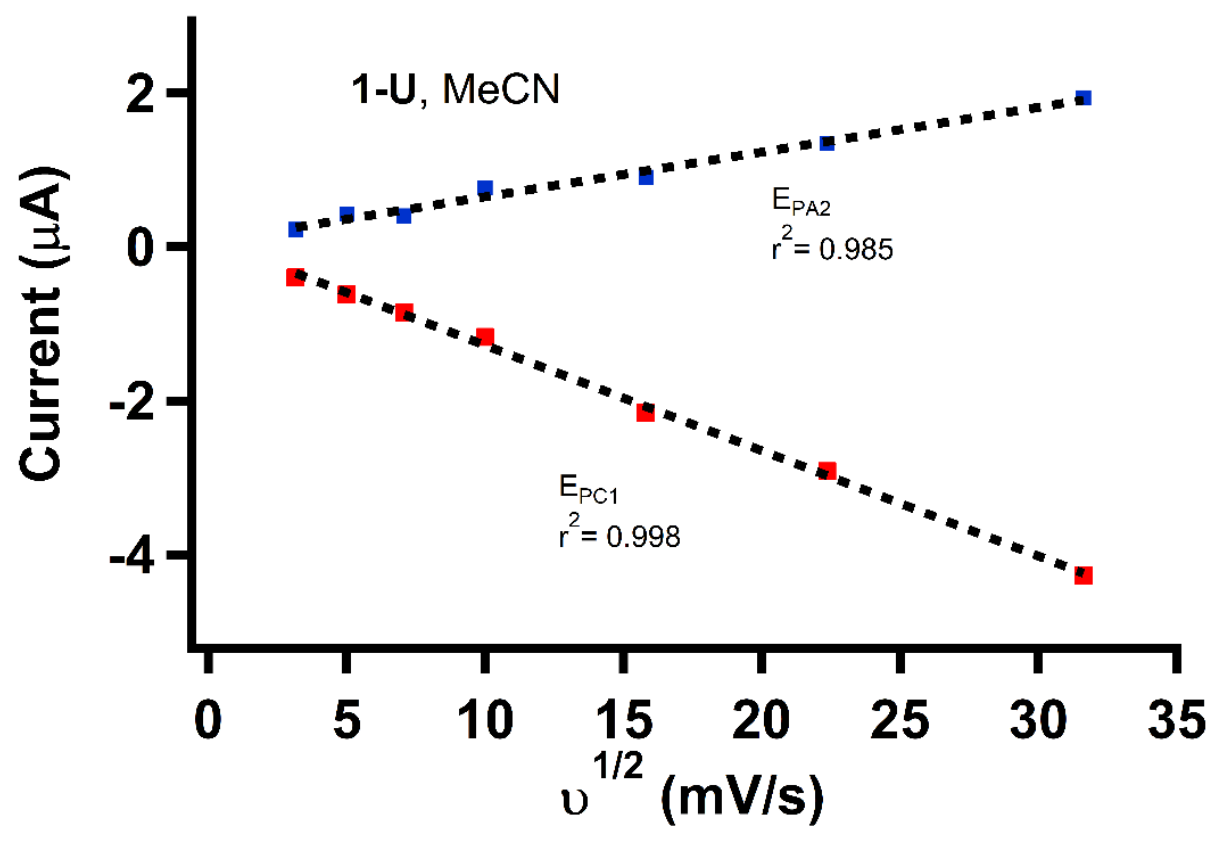

Figure S13. Current vs. square root of scan rate plot for the first oxidation ( $E_{\mathrm{PA} 2}$, blue) and first reduction $\left(\mathrm{E}_{\mathrm{PC} 1}\right.$, red) of $\left[\mathrm{U}(\mathrm{crypt}) \mathrm{I}_{2}\right] \mathrm{I}$ observed when scanning cathodically in MeCN between 10 to $1000 \mathrm{mV} / \mathrm{s}$ scan rate.

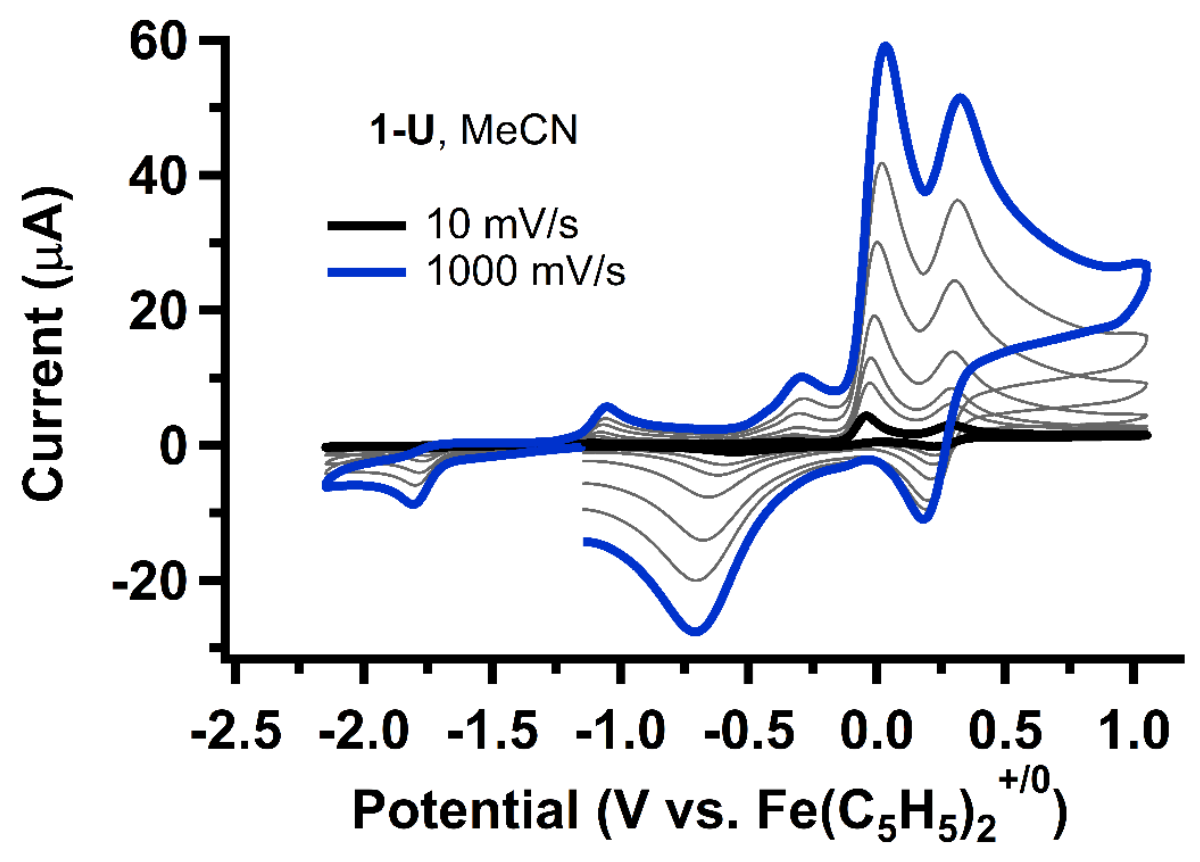

Figure S14. Variable scan rate voltammograms of [U(crypt) $\left.\mathrm{I}_{2}\right] \mathrm{I}$ scanning cathodically in $\mathrm{MeCN}$ from 10 to $1000 \mathrm{mV} / \mathrm{s}$ showing all oxidation and reduction events. 


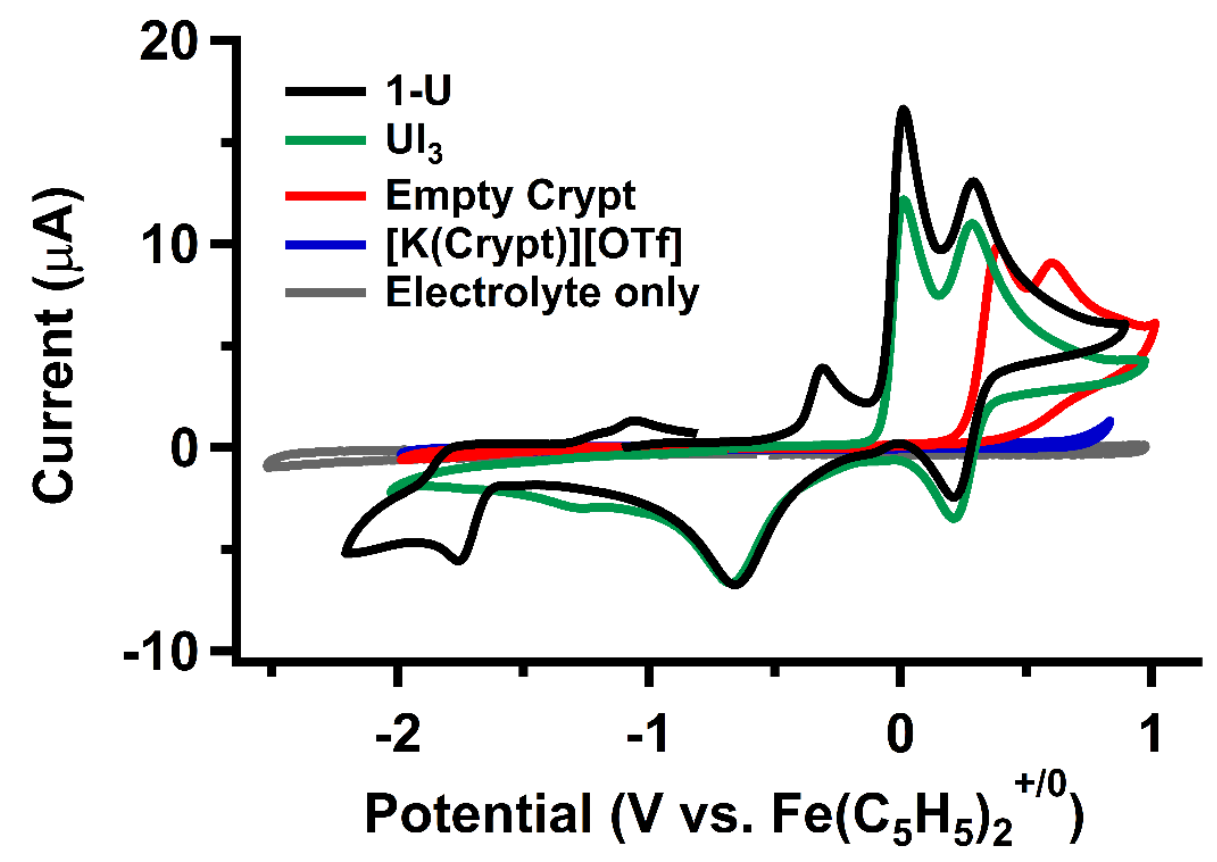

Figure S15. Cyclic voltammograms comparing $3 \mathrm{mM}$ solutions of [U(crypt) $\left.\mathrm{I}_{2}\right] \mathrm{I}$ (1-U, black), $\mathrm{UI}_{3}$ (green), empty cryptand (red), and [K(crypt)[OTf] (blue). Voltammograms were recorded scanning anodically from $\mathrm{OCP}$ in $0.2 \mathrm{M} \mathrm{TBAPF}_{6} \mathrm{MeCN}$ solutions at $100 \mathrm{mV} / \mathrm{s}$ scan rate.
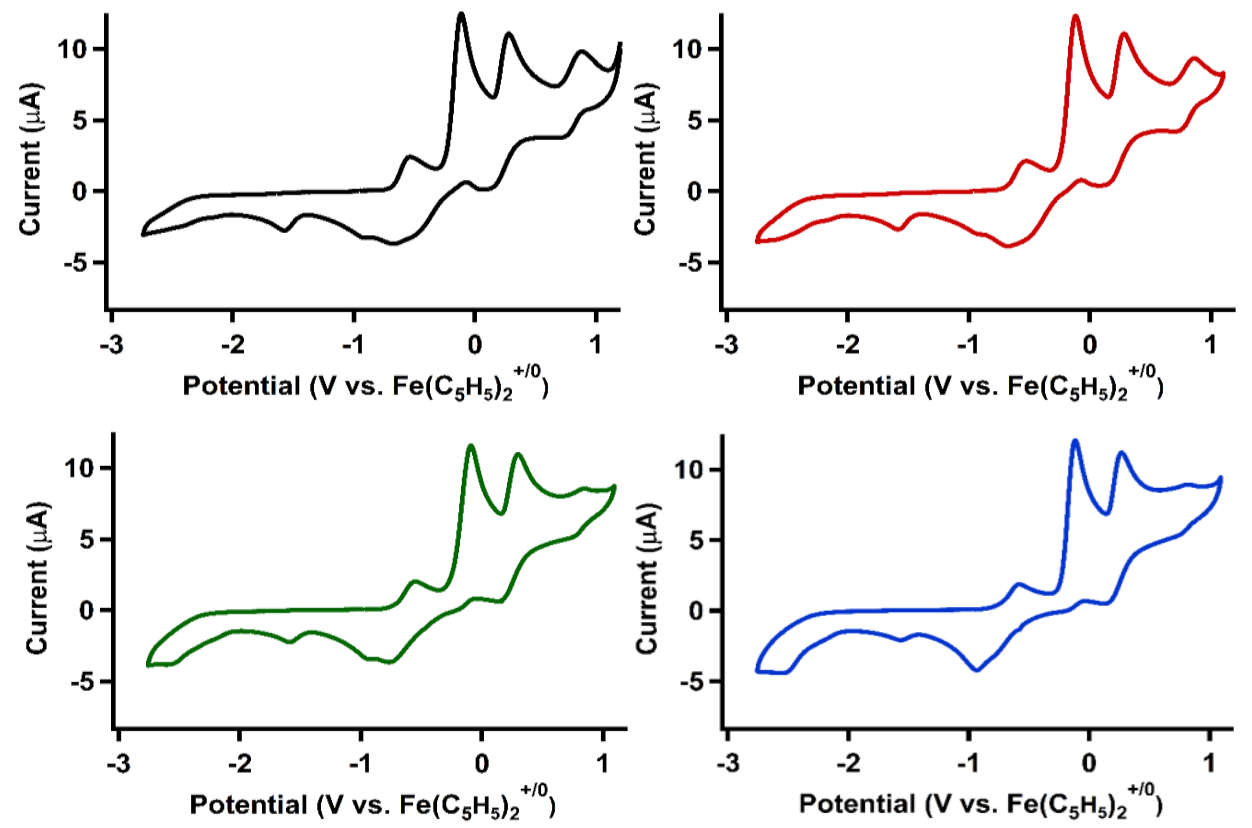

Figure S16. Individual cyclic voltammograms of $\mathbf{1 - U}$ in DMF with increasing concentrations of added water. Voltammograms were recorded using $3 \mathrm{mM}$ analyte in $0.2 \mathrm{M} \mathrm{TBAPF}_{6} \mathrm{DMF}_{\text {. Each }}$ spectrum was recorded scanning anodically with 0 (black), 1 (red), 50 (green), or 250 (blue) equivalents of water 


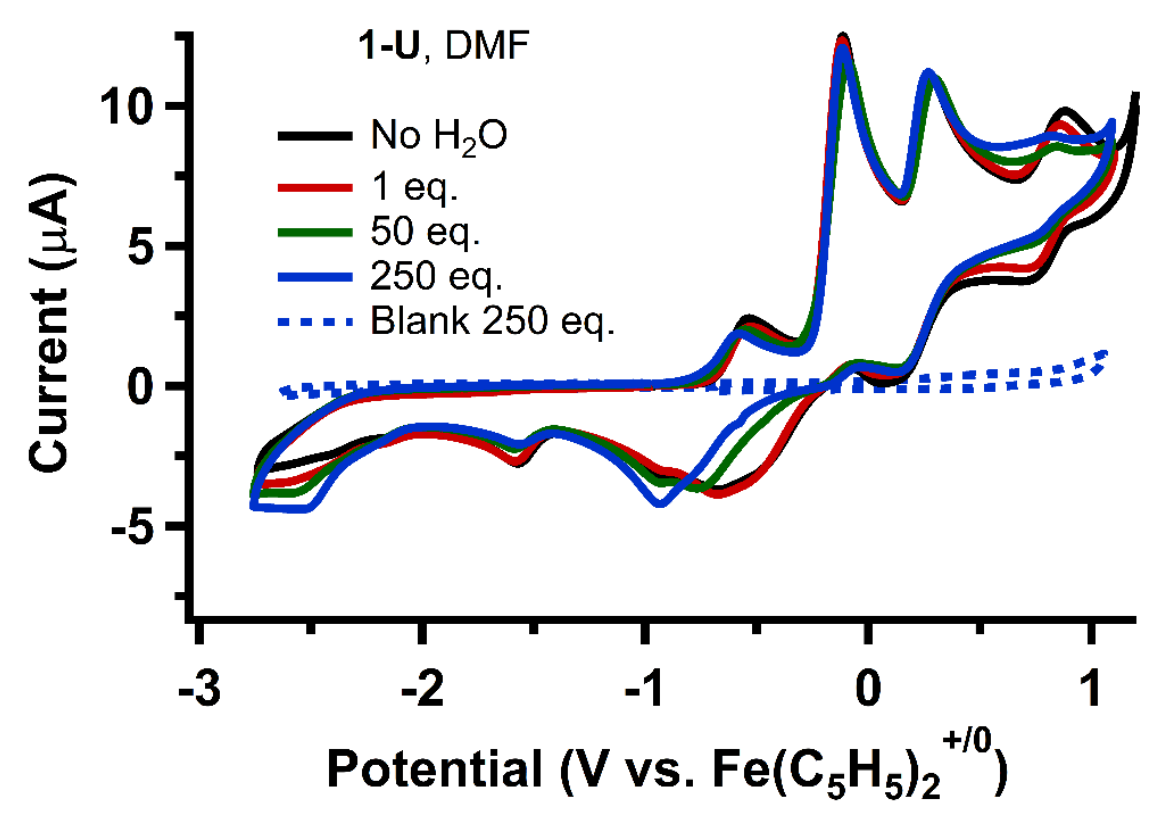

Figure S17. Cyclic voltammograms of 1-U in DMF with increasing concentrations of water in the presence (solid trace) or absence (dotted trace) of $3 \mathrm{mM} \mathrm{1-U}$ in $0.2 \mathrm{M}$ TBAPF $_{6}$ DMF. Each spectrum was recorded scanning anodically with 0 (black), 1 (red), 50 (green), or 250 (blue) equivalents of water.

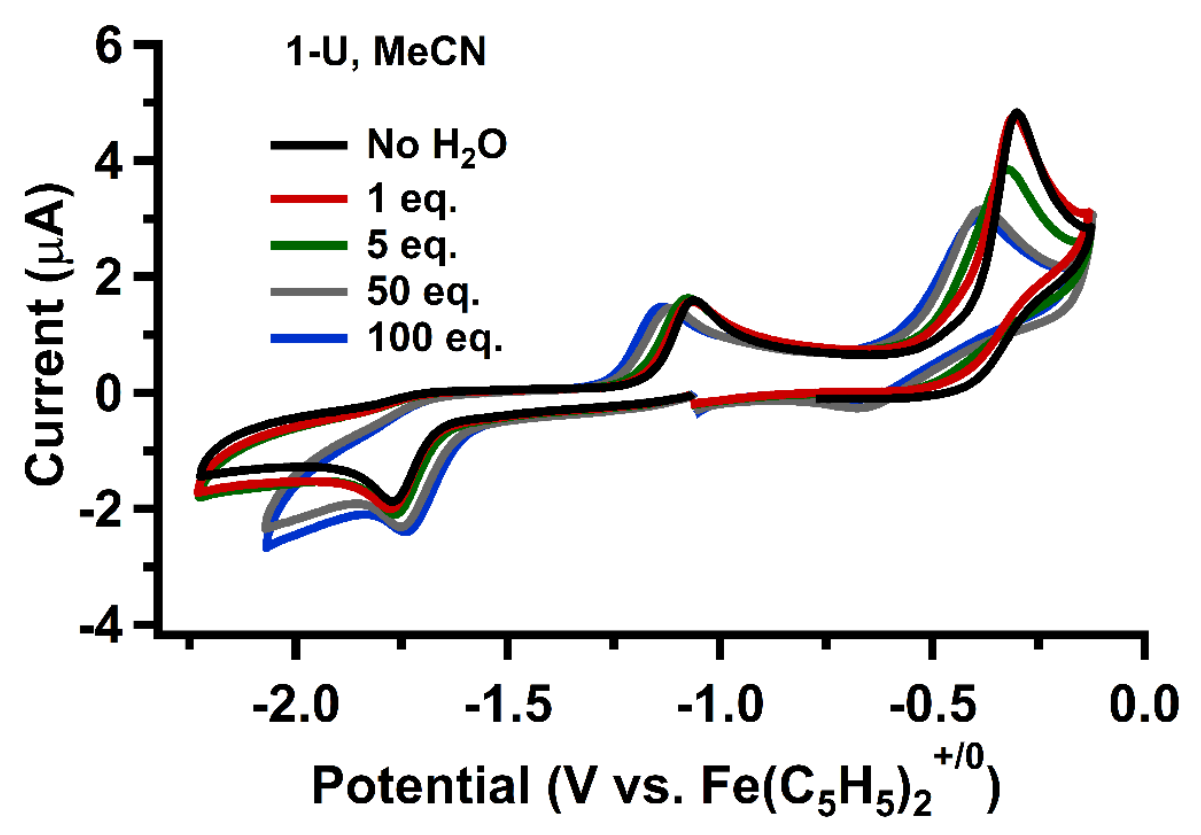

Figure S18. Cyclic voltammograms of $\mathbf{1 - U}$ in $\mathrm{MeCN}$ with increasing concentrations of added water lacking redox events arising from free iodide in solution. Voltammograms are recorded scanning cathodically from OCP with 0 (black), 1 (red), 5 (green), 50 (grey), or 100 (blue) equivalents of water. All measurements were recorded using $3 \mathrm{mM} \mathrm{1-U}$ in a $0.3 \mathrm{M} \mathrm{TBAPF} 6$ electrolyte solution in $\mathrm{MeCN}$ at a $100 \mathrm{mV} / \mathrm{s}$ scan rate. 


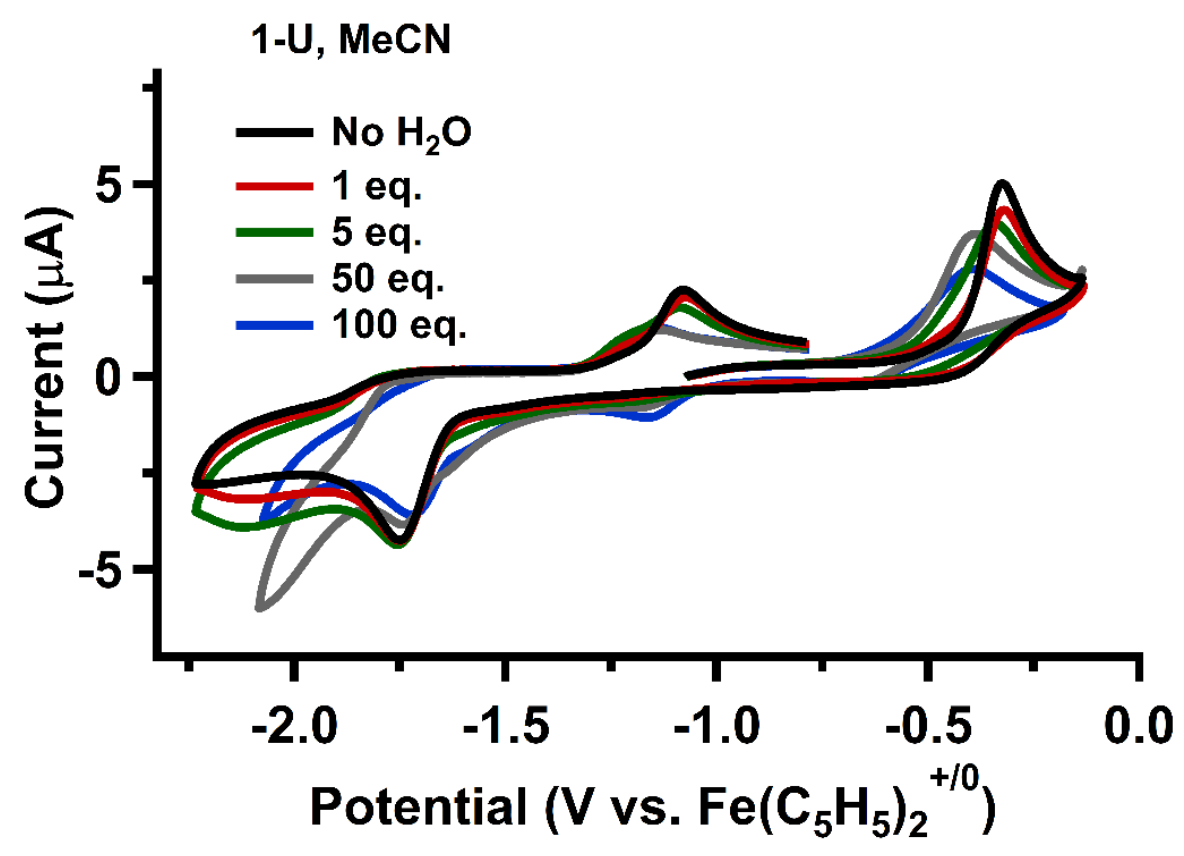

Figure S19. Cyclic voltammograms of $\mathbf{1 - U}$ in $\mathrm{MeCN}$ with increasing concentrations of added water lacking redox events arising from free iodide in solution. Voltammograms are recorded scanning anodically from OCP with 0 (black), 1 (red), 5 (green), 50 (grey), or 100 (blue) equivalents of water. All measurements were recorded using $3 \mathrm{mM} \mathbf{1 - U}$ in a $0.3 \mathrm{M} \mathrm{TBAPF} 6$ electrolyte solution in $\mathrm{MeCN}$ at a $100 \mathrm{mV} / \mathrm{s}$ scan rate.

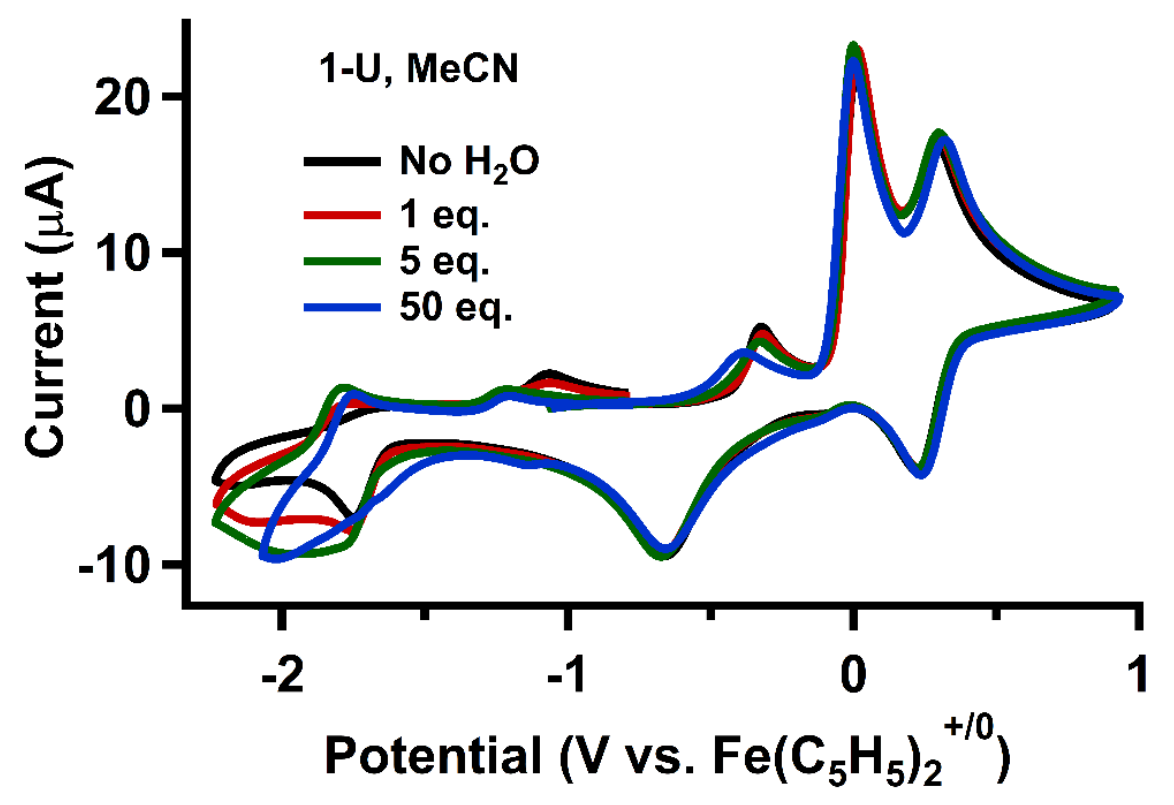

Figure S20. Cyclic voltammograms of $\mathbf{1 - U}$ in $\mathrm{MeCN}$ with increasing concentrations of added water. Voltammograms are recorded scanning anodically from OCP with 0 (black), 1 (red), 5 (green), 50 (blue), or 100 (grey) equivalents of water. All measurements were recorded using 3 $\mathrm{mM} \mathbf{1 - U}$ in a $0.3 \mathrm{M} \mathrm{TBAPF}_{6}$ electrolyte solution in $\mathrm{MeCN}$ at a $100 \mathrm{mV} / \mathrm{s}$ scan rate. 


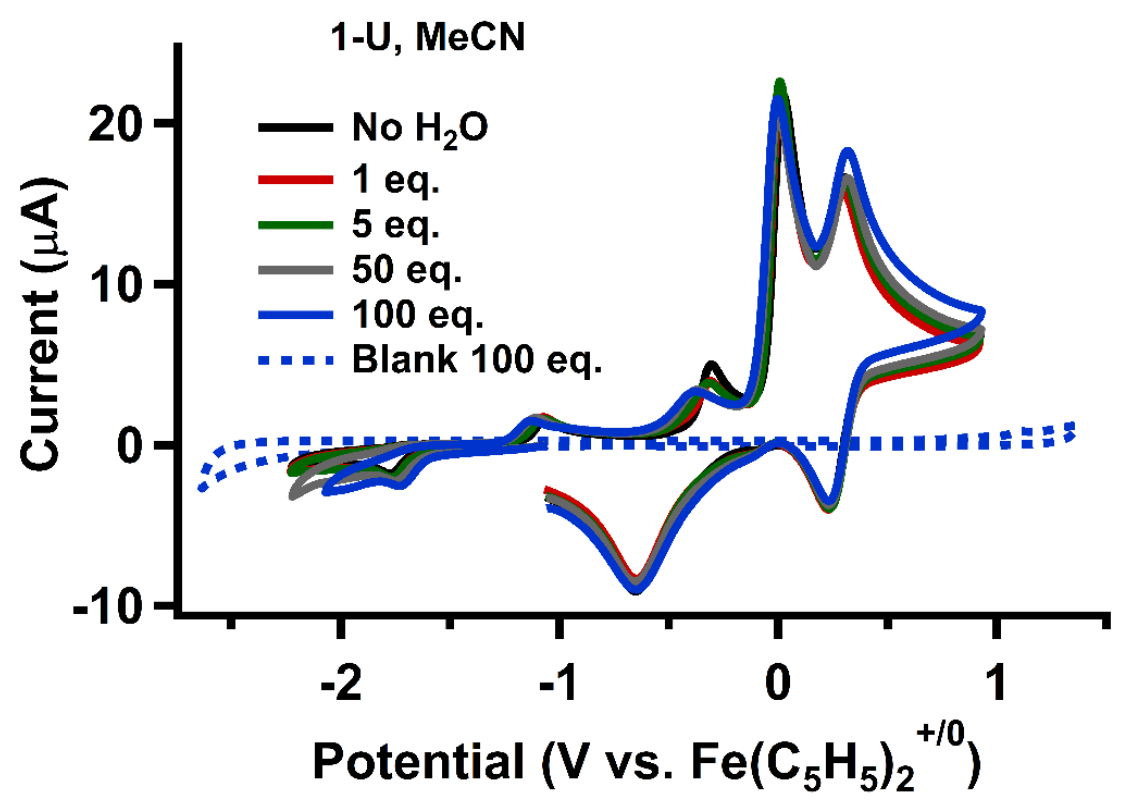

Figure S21. Cyclic voltammograms of 1-U in $\mathrm{MeCN}$ with increasing concentrations of added water. Voltammograms are recorded scanning cathodically in the presence (solid trace) or absence (dotted trace) of 1-U with 0 (black), 1 (red), 5 (green), 50 (grey), or 100 (blue) equivalents of water. All measurements were recorded using $3 \mathrm{mM} \mathrm{1-U}$ in a $0.2 \mathrm{M} \mathrm{TBAPF}_{6}$ electrolyte solution in $\mathrm{MeCN}$ at a $100 \mathrm{mV} / \mathrm{s}$ scan rate.

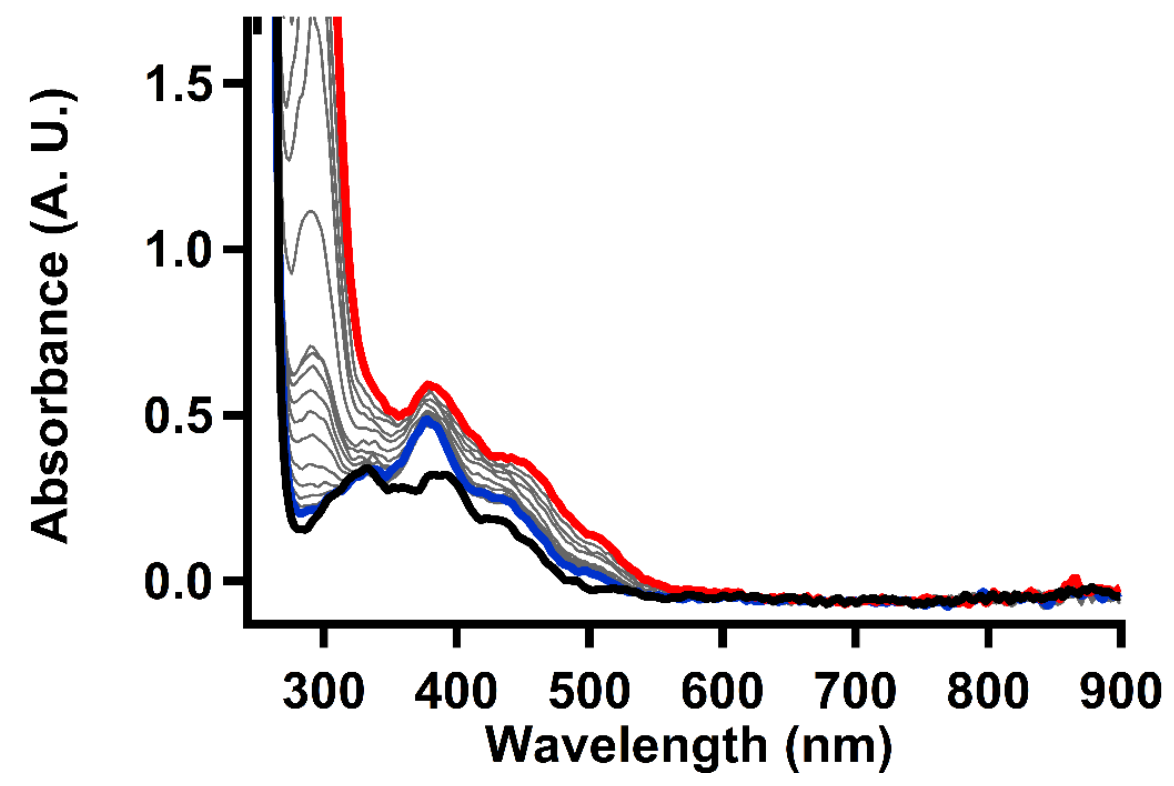

Figure S22. Electronic absorption spectra of $\left[\mathrm{U}(\right.$ crypt $\left.) \mathrm{I}_{2}\right] \mathrm{I}$ in $\mathrm{MeCN}$ after SEC UV-Vis at $-2.0 \mathrm{~V}$ (vs. $\left.\mathrm{Fe}\left(\mathrm{C}_{5} \mathrm{H}_{5}\right)_{2}{ }^{+/ 0}\right)$ applied potential. Spectra were collected periodically over several minutes after current was no longer being passed. Upon reduction, $\left[\mathrm{U}(\right.$ crypt $\left.) \mathrm{I}_{2}\right] \mathrm{I}$ (black trace) is converted to a new species (blue trace), which then further reacts over several minutes to form another species (red). 


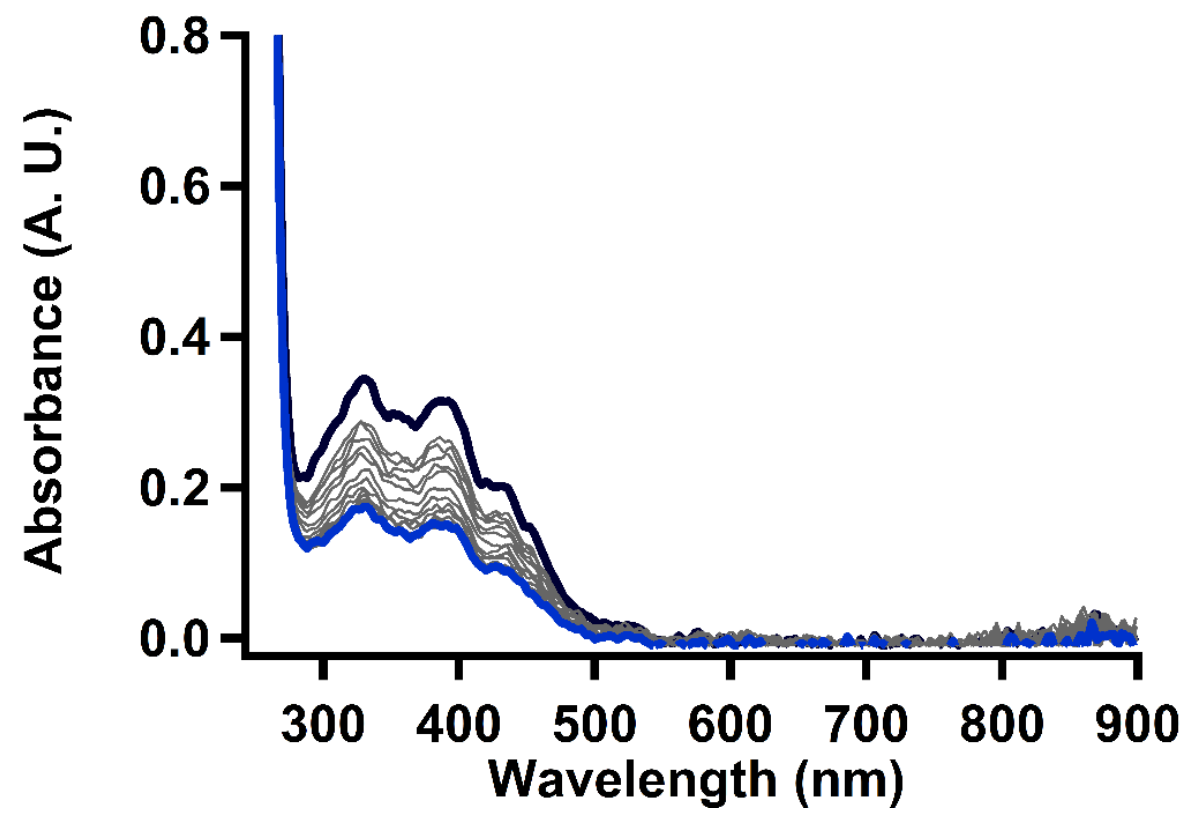

Figure S23. Electronic absorption spectra of [U(crypt) $\left.\mathrm{I}_{2}\right] \mathrm{I}$ in MeCN after SEC UV-Vis at $-0.15 \mathrm{~V}$ (vs. $\left.\mathrm{Fe}\left(\mathrm{C}_{5} \mathrm{H}_{5}\right)_{2}{ }^{+/ 0}\right)$ applied potential. Spectra were collected every 5 seconds until current was no longer being passed. Upon oxidation, all absorbances corresponding to [U(crypt) $\left.\mathrm{I}_{2}\right] \mathrm{I}$ (black trace) decrease in intensity until oxidation is complete (blue trace). The solution becomes completely colorless within several minutes of oxidation.

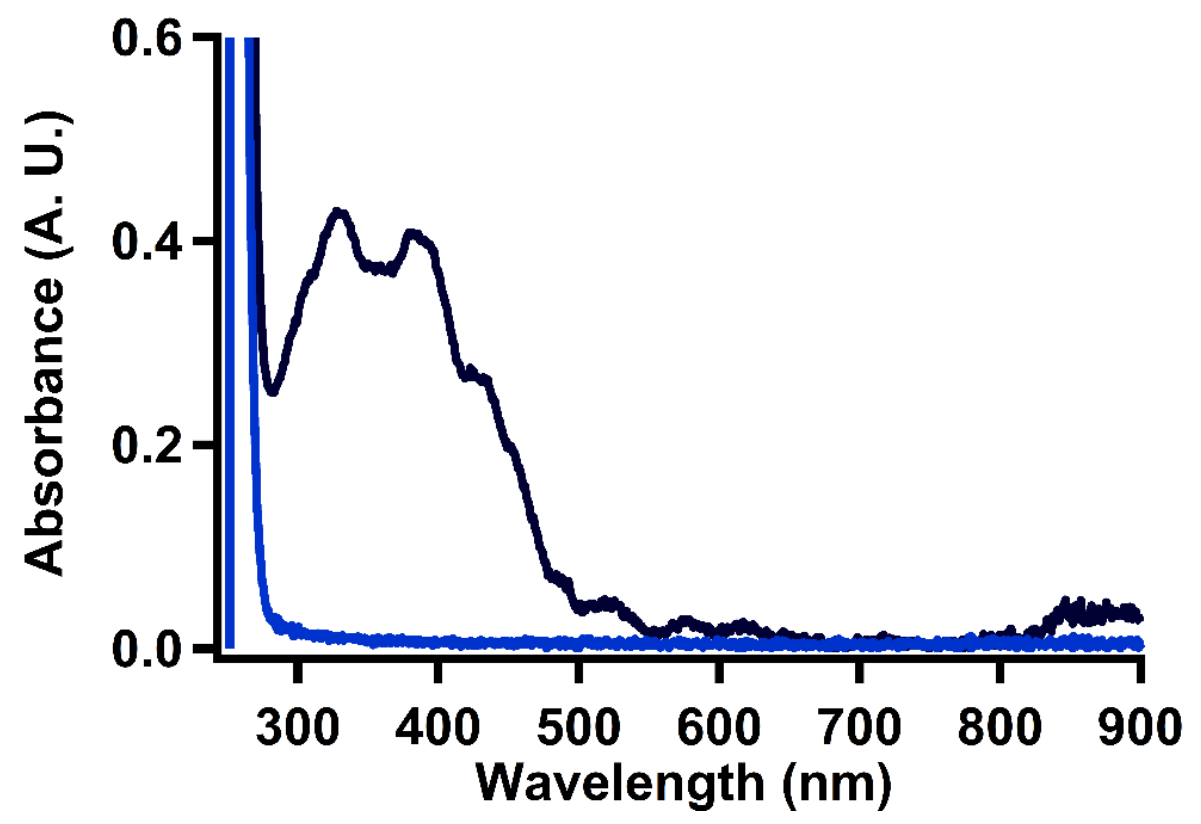

Figure S24. Electronic absorption spectra in $\mathrm{MeCN}$ of $0.20 \mathrm{mM}$ [U(crypt) $\left.\mathrm{I}_{2}\right] \mathrm{I}$ (black trace) and $0.3 \mathrm{mM}$ isolated oxidation product (blue trace) after reaction with 1 eq. $\left[\mathrm{Fe}\left(\mathrm{C}_{5} \mathrm{H}_{5}\right)_{2}\right][\mathrm{BF} 4]$. 
Table S2. Reported U(IV/III) reduction potentials $\left[\mathrm{E}_{1 / 2}\left(\mathrm{U}^{\mathrm{IV} / \mathrm{III}}\right)\right]$ for various uranium complexes including solvent and reference utilized for the measurement.

\begin{tabular}{|c|c|c|c|c|}
\hline Complex & $\underset{\left(\mathbf{U}^{\mathrm{IV} / I I I}\right)^{\dagger}}{\mathbf{E}_{1 / 2}}$ & Solvent & $\begin{array}{l}\text { Reference } \\
\text { electrode }\end{array}$ & $\begin{array}{c}\text { Reference } \\
\quad \#\end{array}$ \\
\hline$(\mathrm{Cp})_{3} \mathrm{UCl}$ & -1.29 & THF & $\mathrm{Ag} / \mathrm{AgCl}$ & 3 \\
\hline$(\mathrm{Cp})_{3} \mathrm{UCl}$ & -1.80 & THF & {$\left[\mathrm{Fe}\left(\mathrm{C}_{5} \mathrm{H}_{5}\right)_{2}\right]^{+/ 0}$} & 4 \\
\hline $\mathrm{U}(\mathbf{C p})_{4}$ & -1.94 & THF & {$\left[\mathrm{Fe}\left(\mathrm{C}_{5} \mathrm{H}_{5}\right)_{2}\right]^{+/ 0}$} & 4 \\
\hline$\left(\mathbf{C p}^{*}\right)_{2} \mathrm{UCl}_{2}$ & -2.18 & THF & {$\left[\mathrm{Fe}\left(\mathrm{C}_{5} \mathrm{H}_{5}\right)_{2}\right]^{+/ 0}$} & 4 \\
\hline$\left(\mathbf{C p}^{i \operatorname{Pr} 4}\right)_{2} \mathrm{UF}_{2}$ & -2.23 & THF & {$\left[\mathrm{Fe}\left(\mathrm{C}_{5} \mathrm{H}_{5}\right)_{2}\right]^{+/ 0}$} & 5 \\
\hline$\left(\mathrm{Cp}^{i \mathrm{Pr} 4}\right)_{2} \mathrm{UCl}_{2}$ & -1.70 & THF & {$\left[\mathrm{Fe}\left(\mathrm{C}_{5} \mathrm{H}_{5}\right)_{2}\right]^{+/ 0}$} & 5 \\
\hline$\left(\mathrm{Cp}^{i \operatorname{Pr} 4}\right)_{2} \mathrm{UBr}_{2}$ & -1.57 & THF & {$\left[\mathrm{Fe}\left(\mathrm{C}_{5} \mathrm{H}_{5}\right)_{2}\right]^{+/ 0}$} & 5 \\
\hline$\left(\mathbf{C} p^{i \mathbf{P r} 4}\right)_{2} \mathbf{U I}_{2}$ & -1.41 & $\mathrm{THF}$ & {$\left[\mathrm{Fe}\left(\mathrm{C}_{5} \mathrm{H}_{5}\right)_{2}\right]^{+/ 0}$} & 5 \\
\hline $\mathrm{UCl}_{4}$ & -1.64 & DMF & $\mathrm{Ag} / \mathrm{Ag}^{+}$ & 6 \\
\hline$[\mathrm{BuMeIm}]_{2}\left[\mathrm{UCl}_{6}\right]$ & -1.99 & {$[\mathrm{BuMeIm}]\left[\mathrm{Tf}_{2} \mathrm{~N}\right]$} & {$\left[\mathrm{Fe}\left(\mathrm{C}_{5} \mathrm{H}_{5}\right)_{2}\right]^{+/ 0}$} & 7 \\
\hline$[\mathrm{BuMePyr}]_{2}\left[\mathrm{UCl}_{6}\right]$ & -2.09 & {$[\mathrm{BuMePyr}]\left[\mathrm{Tf}_{2} \mathrm{~N}\right]$} & {$\left[\mathrm{Fe}\left(\mathrm{C}_{5} \mathrm{H}_{5}\right)_{2}\right]^{+/ 0}$} & 7 \\
\hline$\left[\mathrm{BuMe}_{2} \mathrm{Im}\right]_{2}\left[\mathrm{UCl}_{6}\right]$ & -2.16 & {$\left[\mathrm{BuMe}_{2} \mathrm{Im}\right]\left[\mathrm{Tf}_{2} \mathrm{~N}\right]$} & {$\left[\mathrm{Fe}\left(\mathrm{C}_{5} \mathrm{H}_{5}\right)_{2}\right]^{+/ 0}$} & 7 \\
\hline$\left[\mathrm{MeBu}_{3} \mathrm{~N}\right]_{2}\left[\mathrm{UCl}_{6}\right]$ & -2.21 & {$\left[\mathrm{MeBu}_{3} \mathrm{~N}\right]\left[\mathrm{Tf}_{2} \mathrm{~N}\right]$} & {$\left[\mathrm{Fe}\left(\mathrm{C}_{5} \mathrm{H}_{5}\right)_{2}\right]^{+/ 0}$} & 7 \\
\hline $\mathrm{U}(\mathbf{a c a c})_{4}$ & -2.61 & THF & $\mathrm{Ag} / \mathrm{AgClO}_{4}$ & 8 \\
\hline$(\mathbf{C p} *)_{2} \mathbf{U I}(\mathrm{THF})$ & -1.12 & THF & {$\left[\mathrm{Fe}\left(\mathrm{C}_{5} \mathrm{H}_{5}\right)_{2}\right]^{+/ 0}$} & 9 \\
\hline$(\mathrm{PNP})_{2} \mathrm{UCl}_{2}$ & -2.19 & THF & {$\left[\mathrm{Fe}\left(\mathrm{C}_{5} \mathrm{H}_{5}\right)_{2}\right]^{+/ 0}$} & 9 \\
\hline$(\mathbf{P N P})_{2} \mathrm{UI}$ & -1.47 & THF & {$\left[\mathrm{Fe}\left(\mathrm{C}_{5} \mathrm{H}_{5}\right)_{2}\right]^{+/ 0}$} & 9 \\
\hline$\left[\mathbf{E t}_{4} \mathbf{N}\right]_{4}\left[\mathbf{U}(\mathbf{N C S})_{8}\right]$ & $-1.38^{\mathrm{a}}$ & $\mathrm{MeCN}$ & {$\left[\mathrm{Fe}\left(\mathrm{C}_{5} \mathrm{H}_{5}\right)_{2}\right]^{+/ 0}$} & 10 \\
\hline$\left[\mathrm{Et}_{4} \mathrm{~N}\right]\left[\mathrm{U}(\mathrm{NCS})_{5}\left(\mathrm{bipy}_{2}\right]\right.$ & $-0.80^{\mathrm{a}}$ & $\mathrm{MeCN}$ & {$\left[\mathrm{Fe}\left(\mathrm{C}_{5} \mathrm{H}_{5}\right)_{2}\right]^{+/ 0}$} & 10 \\
\hline$\left[\mathrm{U}(\mathrm{dmso})_{8}\right]\left(\mathrm{ClO}_{4}\right)_{4}$ & $\begin{array}{l}-0.93^{\mathrm{a}} \\
-0.82^{\mathrm{b}}\end{array}$ & DMSO & {$\left[\mathrm{Fe}\left(\mathrm{C}_{5} \mathrm{H}_{5}\right)_{2}\right]^{+/ 0}$} & 11 \\
\hline [UI I $_{2}{ }^{\mathrm{Me}}$ napthquinolen $\left.)_{2}\right]$ & -2.16 & pyridine & {$\left[\mathrm{Fe}\left(\mathrm{C}_{5} \mathrm{H}_{5}\right)_{2}\right]^{+/ 0}$} & 12 \\
\hline$\left[\left(\left({ }^{t-\mathrm{Bu}}, t-\mathrm{Bu} \mathrm{ArO}\right)\right)_{4}\right.$ cyclen $\left.) \mathrm{U}\right]$ & -3.10 & THF & {$\left[\mathrm{Fe}\left(\mathrm{C}_{5} \mathrm{H}_{5}\right)_{2}\right]^{+/ 0}$} & 13 \\
\hline$\left(\mathbf{C p}^{*}\right)_{2} \mathrm{U}\left(\mathrm{CH}_{2} \mathbf{P h}\right)$ & -1.95 & THF & {$\left[\mathrm{Fe}\left(\mathrm{C}_{5} \mathrm{H}_{5}\right)_{2}\right]^{+/ 0}$} & 14 \\
\hline$(\mathbf{C p} *) \mathbf{U}\left(\mathrm{CH}_{2} \mathbf{P h}\right)_{3}$ & -2.00 & THF & {$\left[\mathrm{Fe}\left(\mathrm{C}_{5} \mathrm{H}_{5}\right)_{2}\right]^{+/ 0}$} & 14 \\
\hline$\left(\mathrm{Cp}^{*}\right)_{2} \mathrm{UCH}_{3}$ & -2.41 & THF & {$\left[\mathrm{Fe}\left(\mathrm{C}_{5} \mathrm{H}_{5}\right)_{2}\right]^{+/ 0}$} & 14 \\
\hline$\left(\mathrm{Cp}^{*}\right)_{2} \mathrm{UCH}_{3}\left(\mathrm{SO}_{3} \mathrm{CF}_{3}\right)$ & -1.83 & THF & {$\left[\mathrm{Fe}\left(\mathrm{C}_{5} \mathrm{H}_{5}\right)_{2}\right]^{+/ 0}$} & 14 \\
\hline $\begin{array}{c}\left(\mathrm{Cp}^{*}\right)_{2} \mathrm{U}\left(\mathrm{SO}_{3} \mathrm{CF}_{3}\right)\left[\eta^{2}\left(\mathrm{~N}, \mathrm{~N}^{\prime}\right)-\right. \\
\left.\mathrm{CH}_{3} \mathrm{NN}=\mathrm{CPh}_{2}\right]\end{array}$ & -2.01 & THF & {$\left[\mathrm{Fe}\left(\mathrm{C}_{5} \mathrm{H}_{5}\right)_{2}\right]^{+/ 0}$} & 14 \\
\hline $\begin{array}{c}\left(\mathrm{Cp}^{*}\right)_{2} \mathrm{UCl}\left[\eta^{2}\left(\mathrm{~N}, \mathrm{~N}^{\prime}\right)-\right. \\
\left.\mathrm{CH}_{3} \mathrm{NN}=\mathrm{CPh}_{2}\right]\end{array}$ & -2.29 & THF & {$\left[\mathrm{Fe}\left(\mathrm{C}_{5} \mathrm{H}_{5}\right)_{2}\right]^{+/ 0}$} & 14 \\
\hline $\begin{array}{c}\left(\mathrm{Cp}^{*}\right)_{2} \mathrm{U}\left[\eta^{2}\left(\mathrm{~N}, \mathrm{~N}^{\prime}\right)-\right. \\
\left.\mathrm{CH}_{3} \mathrm{NN}=\mathrm{CPh}_{2}\right]_{2}\end{array}$ & -2.78 & THF & {$\left[\mathrm{Fe}\left(\mathrm{C}_{5} \mathrm{H}_{5}\right)_{2}\right]^{+/ 0}$} & 14 \\
\hline $\begin{array}{c}\left(\mathrm{Cp}^{*}\right)_{2} \mathrm{U}\left[\eta^{2}\left(\mathrm{~N}, \mathrm{~N}^{\prime}\right)-\right. \\
\left.\mathrm{CH}_{2}(\mathrm{Ph}) \mathrm{NN}=\mathrm{CPh}_{2}\right]_{2}\end{array}$ & -2.56 & THF & {$\left[\mathrm{Fe}\left(\mathrm{C}_{5} \mathrm{H}_{5}\right)_{2}\right]^{+/ 0}$} & 14 \\
\hline$\left(\mathrm{Cp}^{*}\right)_{2} \mathrm{U}\left(-\mathrm{N}=\mathrm{CPh}_{2}\right)_{2}$ & -2.50 & THF & {$\left[\mathrm{Fe}\left(\mathrm{C}_{5} \mathrm{H}_{5}\right)_{2}\right]^{+/ 0}$} & 14 \\
\hline$\left(\mathrm{Cp}^{*}\right)_{2} \mathrm{U}\left[-\mathrm{N}=\mathrm{C}(\mathrm{Ph})\left(\mathrm{CH}_{3}\right)\right]_{2}$ & -2.68 & THF & {$\left[\mathrm{Fe}\left(\mathrm{C}_{5} \mathrm{H}_{5}\right)_{2}\right]^{+/ 0}$} & 14 \\
\hline$\left.(\mathbf{C p})_{2}\right)_{2} \mathrm{U}\left[-\mathrm{N}=\mathrm{C}(\mathrm{Ph})\left(\mathrm{CH}_{2} \mathrm{Ph}\right)\right]_{2}$ & -2.57 & THF & {$\left[\mathrm{Fe}\left(\mathrm{C}_{5} \mathrm{H}_{5}\right)_{2}\right]^{+/ 0}$} & 14 \\
\hline$\left(\mathrm{Cp}^{*}\right)_{2} \mathrm{U}\left(=\mathrm{N}-2,4,6-{ }^{t-\mathrm{Bu} 3} \mathrm{C}_{6} \mathrm{H}_{2}\right)$ & -2.61 & THF & {$\left[\mathrm{Fe}\left(\mathrm{C}_{5} \mathrm{H}_{5}\right)_{2}\right]^{+/ 0}$} & 14 \\
\hline
\end{tabular}

Reported in units of volts.

${ }^{\text {a Reported }} \mathrm{E}_{\mathrm{pc}}$ for irreversible $\mathrm{U}^{\mathrm{IV} / \mathrm{III}}$ reduction.

${ }^{b}$ Reported $\mathrm{E}_{\mathrm{pa}}$ for irreversible $\mathrm{U}^{\mathrm{III} / \mathrm{IV}}$ oxidation. 
Table S2 continued. Reported U(IV/III) reduction potentials $\left[\mathrm{E}_{1 / 2}\left(\mathrm{U}^{\mathrm{IV} / \mathrm{III}}\right)\right]$ for various uranium complexes including solvent and reference utilized for the measurement.

\begin{tabular}{|c|c|c|c|c|}
\hline Complex & $\underset{\left(\mathbf{U}^{\mathrm{IV} / \mathrm{III}}\right)^{\dagger}}{\mathbf{E}_{\mathbf{1} / \mathbf{2}}}$ & Solvent & $\begin{array}{l}\text { Reference } \\
\text { electrode }\end{array}$ & $\begin{array}{c}\text { Reference } \\
\#\end{array}$ \\
\hline$\left(\mathrm{Cp}^{*}\right)_{2} \mathrm{U}\left[-\mathrm{N}=\mathrm{C}\left(\mathrm{CH}_{3}\right)\left(2-\mathrm{F}-\mathrm{C}_{6} \mathrm{H}_{4}\right)\right]_{2}$ & -2.62 & THF & {$\left[\mathrm{Fe}\left(\mathrm{C}_{5} \mathrm{H}_{5}\right)_{2}\right]^{+/ 0}$} & 15 \\
\hline$(\mathrm{Cp} *)_{2} \mathrm{U}\left[-\mathrm{N}=\mathrm{C}\left(\mathrm{CH}_{3}\right)\left(3-\mathrm{F}-\mathrm{C}_{6} \mathrm{H}_{4}\right)\right]_{2}$ & -2.54 & $\mathrm{THF}$ & {$\left[\mathrm{Fe}\left(\mathrm{C}_{5} \mathrm{H}_{5}\right)_{2}\right]^{+/ 0}$} & 15 \\
\hline$\left(\mathrm{Cp}^{*}\right)_{2} \mathrm{U}\left[-\mathrm{N}=\mathrm{C}\left(\mathrm{CH}_{3}\right)\left(4-\mathrm{F}-\mathrm{C}_{6} \mathrm{H}_{4}\right)\right]_{2}$ & -2.64 & $\mathrm{THF}$ & {$\left[\mathrm{Fe}\left(\mathrm{C}_{5} \mathrm{H}_{5}\right)_{2}\right]^{+/ 0}$} & 15 \\
\hline$\left(\mathrm{Cp}^{*}\right)_{2} \mathrm{U}\left[-\mathrm{N}=\mathrm{C}\left(\mathrm{CH}_{3}\right)\left(2,6-\mathrm{F}_{2}-\mathrm{C}_{6} \mathrm{H}_{3}\right)\right]_{2}$ & -2.82 & THF & {$\left[\mathrm{Fe}\left(\mathrm{C}_{5} \mathrm{H}_{5}\right)_{2}\right]^{+/ 0}$} & 15 \\
\hline$\left(\mathrm{Cp}^{*}\right)_{2} \mathrm{U}\left[-\mathrm{N}=\mathrm{C}\left(\mathrm{CH}_{3}\right)\left(3,5-\mathrm{F}_{2}-\mathrm{C}_{6} \mathrm{H}_{3}\right)\right]_{2}$ & -2.46 & THF & {$\left[\mathrm{Fe}\left(\mathrm{C}_{5} \mathrm{H}_{5}\right)_{2}\right]^{+/ 0}$} & 15 \\
\hline 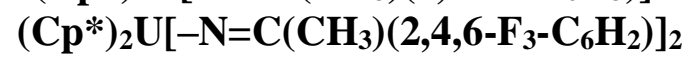 & -2.80 & THF & {$\left[\mathrm{Fe}\left(\mathrm{C}_{5} \mathrm{H}_{5}\right)_{2}\right]^{+/ 0}$} & 15 \\
\hline$\left(\mathrm{Cp}^{*}\right)_{2} \mathrm{U}\left[-\mathrm{N}=\mathrm{C}\left(\mathrm{CH}_{3}\right)\left(\mathbf{3 , 4 , 5}-\mathrm{F}_{3}-\mathrm{C}_{6} \mathrm{H}_{2}\right)\right]_{2}$ & -2.48 & THF & {$\left[\mathrm{Fe}\left(\mathrm{C}_{5} \mathrm{H}_{5}\right)_{2}\right]^{+/ 0}$} & 15 \\
\hline$\left(\mathrm{Cp}^{*}\right)_{2} \mathrm{U}\left[-\mathrm{N}=\mathrm{C}\left(\mathrm{CH}_{3}\right)\left(\mathrm{C}_{6} \mathrm{~F}_{5}\right)\right]_{2}$ & -2.34 & THF & {$\left[\mathrm{Fe}\left(\mathrm{C}_{5} \mathrm{H}_{5}\right)_{2}\right]^{+/ 0}$} & 15 \\
\hline
\end{tabular}

Reported in units of volts.

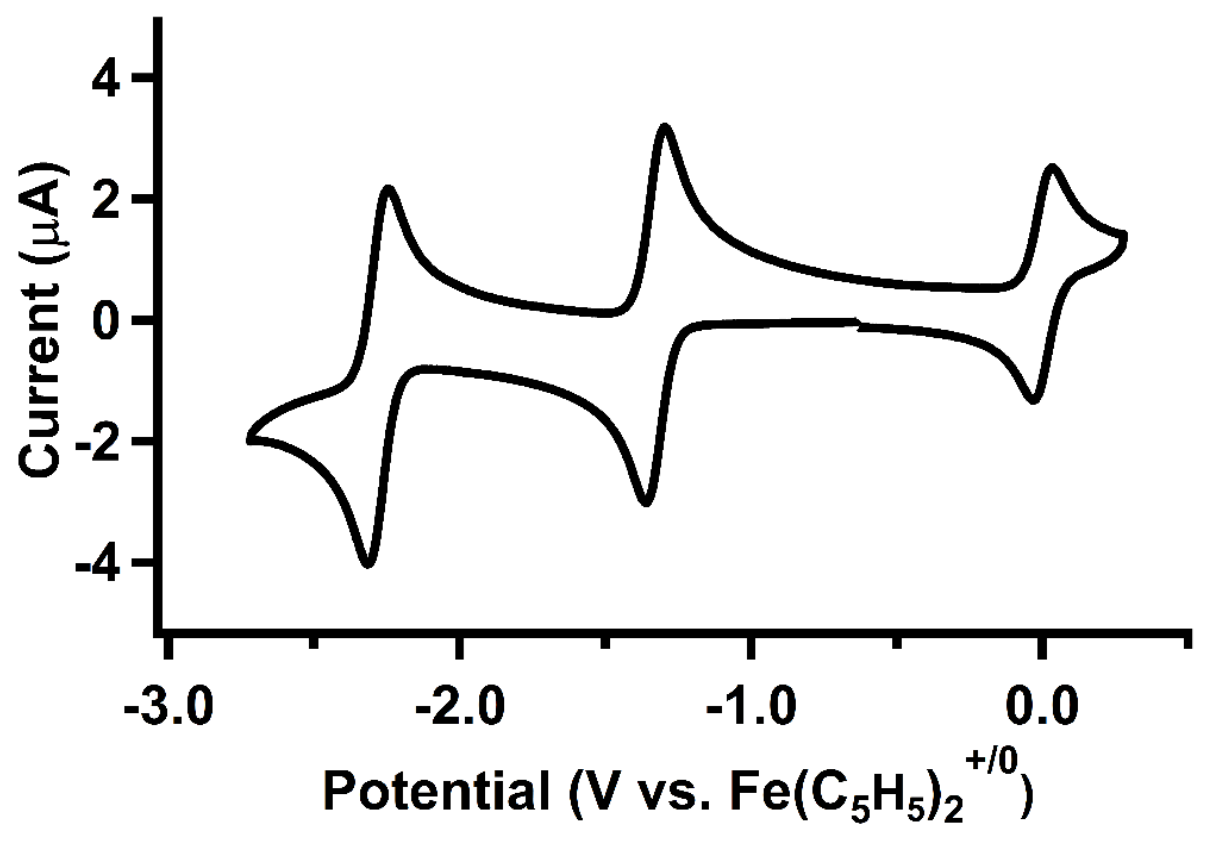

Figure S25. Cyclic Voltammogram of $\left[\mathrm{Co}\left(\mathrm{C}_{5} \mathrm{H}_{5}\right)_{2}\right]\left[\mathrm{PF}_{6}\right]$ under $\mathrm{N}_{2}$ atmosphere in DMF. The Co ${ }^{\text {III/II }}$ couple is centered at $\mathrm{E}_{1 / 2}=-1.33 \mathrm{~V}$. The reversible couple centered at $0.0 \mathrm{~V}$ corresponds to $\mathrm{Fe}\left(\mathrm{C}_{5} \mathrm{H}_{5}\right)_{2}{ }^{+/ 0}$ internal standard. 


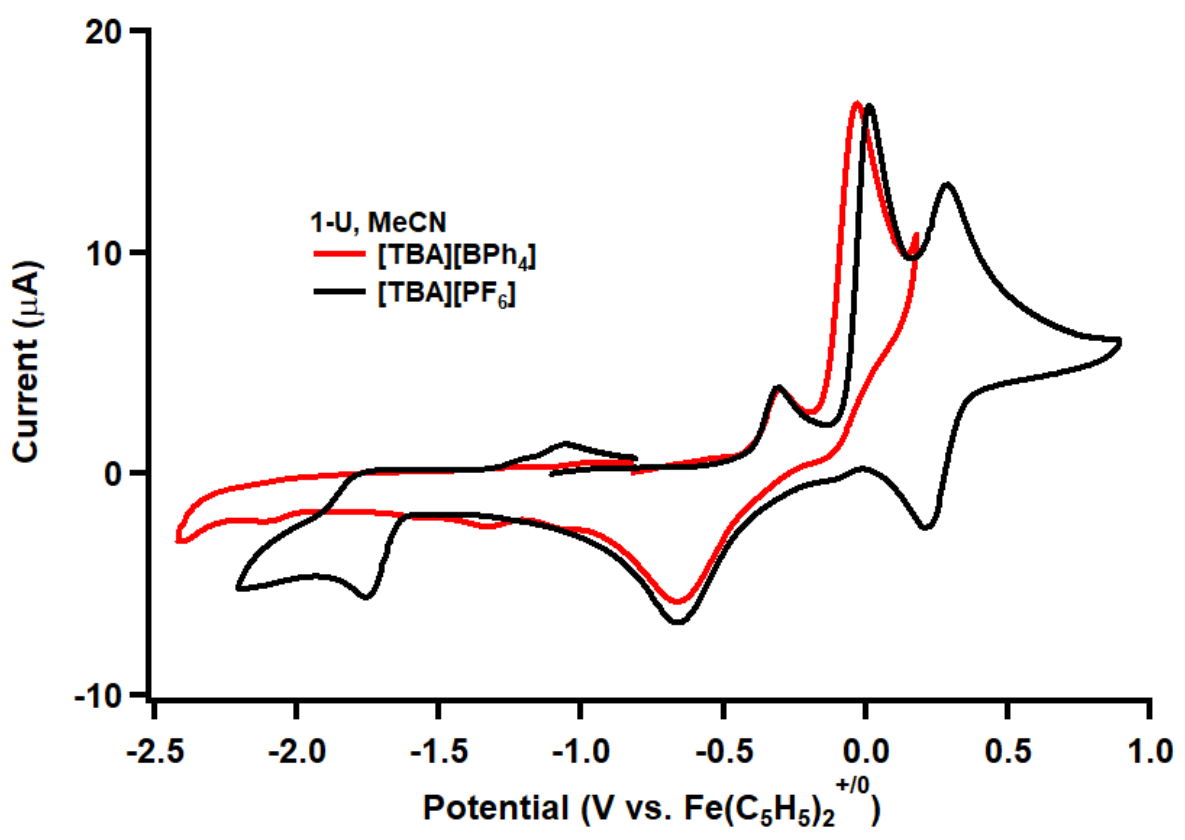

Figure S26. Cyclic Voltammograms of 1-U in MeCN scanning anodically. Voltammograms are shown using $\mathrm{TBAPF}_{6}$ (black) or $\mathrm{TBABPh}_{4}$ (red) electrolyte. The first oxidation remains similar between the electrolytes, however the reduction observed at ca. $-1.75 \mathrm{~V}$ with $\mathrm{TBAPF}_{6}$ is not present when $\mathrm{TBABPh}_{4}$ is used.

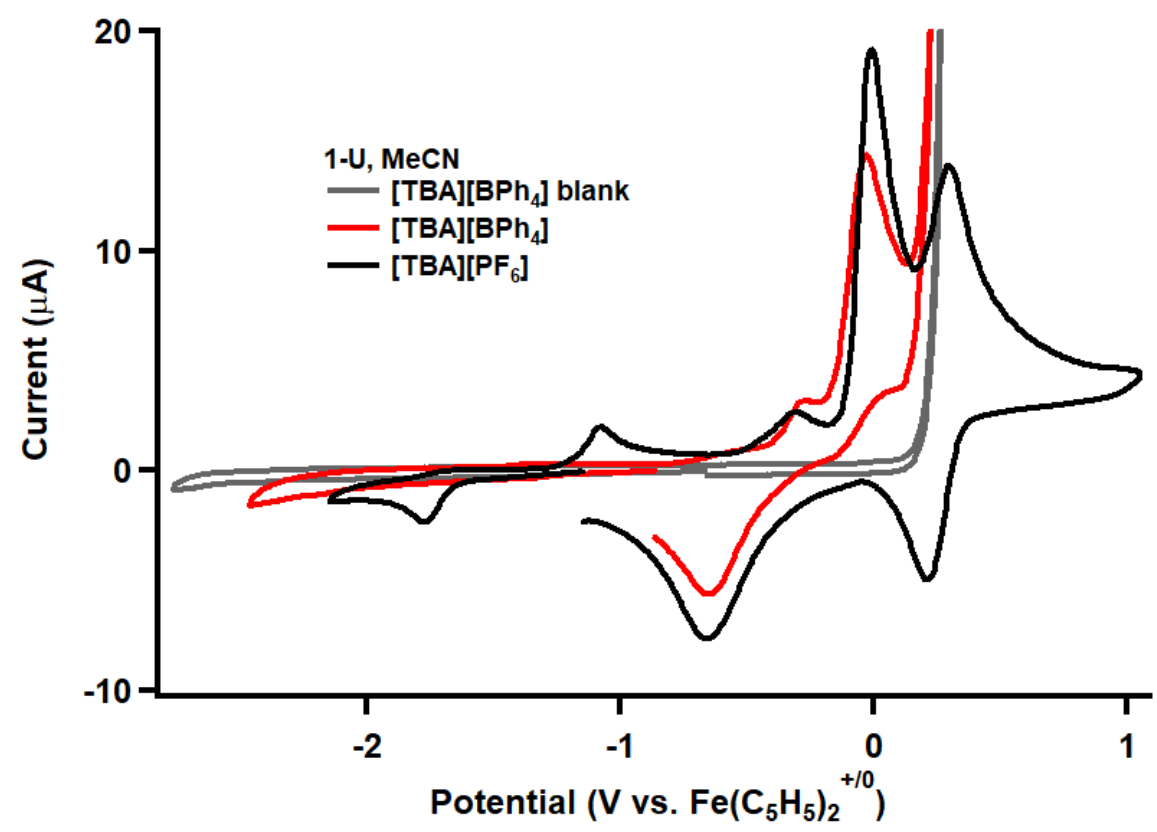

Figure S27. Cyclic Voltammograms of 1-U in MeCN scanning cathodically. Voltammograms are shown using $\mathrm{TBAPF}_{6}$ (black) or $\mathrm{TBABPh}_{4}$ (red) electrolyte. The first oxidation is shifted positive with $\mathrm{BPh}_{4}$ electrolyte, however the reductions appearing negative of OCP with $\mathrm{TBAPF}_{6}$ is not present when $\mathrm{TBABPh}_{4}$ is used. 
X-ray Data Collection, Structure Solution and Refinement for $[\mathrm{U}(\mathrm{crypt})(\mathrm{MeCN}) \mathrm{I}][\mathrm{I}]_{2}, \mathbf{4 - U}$.

A dichroic brown-green crystal of approximate dimensions $0.106 \times 0.188 \times 0.291 \mathrm{~mm}$ was mounted in a cryoloop and transferred to a Bruker SMART APEX II diffractometer. The APEX $2^{16}$ program package was used to determine the unit-cell parameters and for data collection (30 sec/frame scan time for a sphere of diffraction data). The raw frame data was processed using SAINT $^{17}$ and SADABS ${ }^{18}$ to yield the reflection data file. Subsequent calculations were carried out using the SHELXTL ${ }^{19}$ program. The diffraction symmetry was $\mathrm{mmm}$ and the systematic absences were consistent with the orthorhombic space group Pccn that was later determined to be correct.

The structure was solved by dual space methods and refined on $\mathrm{F}^{2}$ by full-matrix least-squares techniques. The analytical scattering factor ${ }^{20}$ for neutral atoms were used throughout the analysis. Hydrogen atoms were included using a riding model. One non-coordinated iodide was disordered and was modeled as a two-part (1:1) disorder.

Least-squares analysis yielded $w \mathrm{R} 2=0.0522$ and Goof $=1.021$ for 300 variables refined against 8990 data $(0.70 \AA), \mathrm{R} 1=0.0265$ for those 7708 data with $\mathrm{I}>2.0 \sigma(\mathrm{I})$.

Table S3. Crystal data and structure refinement for $[\mathrm{U}(\mathrm{crypt})(\mathrm{MeCN}) \mathrm{I}][\mathrm{I}]_{2}$, 4-U.

Identification code

Empirical formula

Formula weight

Temperature

Wavelength

Crystal system

Space group

Unit cell dimensions

Volume

$\mathrm{Z}$

Density (calculated)

Absorption coefficient

$\mathrm{F}(000)$

Crystal color

Crystal size

Theta range for data collection dnh89 (Dan Huh)

$\mathrm{C}_{20} \mathrm{H}_{39} \mathrm{I}_{3} \mathrm{~N}_{3} \mathrm{O}_{6} \mathrm{U}$

1036.27

88(2) K

$0.71073 \AA$

Orthorhombic

Pcen

$\mathrm{a}=31.9678(14) \AA \quad \alpha=90^{\circ}$.

$\mathrm{b}=13.0972(6) \AA \quad \beta=90^{\circ}$.

$\mathrm{c}=14.1365(6) \AA \quad \gamma=90^{\circ}$.

5918.8(5) $\AA^{3}$

8

$2.326 \mathrm{Mg} / \mathrm{m}^{3}$

$8.650 \mathrm{~mm}^{-1}$

3832

brown

$0.291 \times 0.188 \times 0.106 \mathrm{~mm}^{3}$

1.274 to $30.524^{\circ}$ 
Index ranges

Reflections collected

Independent reflections

Completeness to theta $=25.500^{\circ}$

Absorption correction

Max. and min. transmission

Refinement method

Data / restraints / parameters

Goodness-of-fit on $\mathrm{F}^{2}$

Final $\mathrm{R}$ indices $[\mathrm{I}>2 \operatorname{sigma}(\mathrm{I})=7708$ data $]$

$\mathrm{R}$ indices (all data, $0.70 \AA$ )

Largest diff. peak and hole $-43<=\mathrm{h}<=44,-18<=\mathrm{k}<=18,-19<=1<=20$

59636

8990 [R(int) $=0.0437]$

$100.0 \%$

Numerical or multi-scan

0.4330 and 0.2195

Full-matrix least-squares on $\mathrm{F}^{2}$

8990 / 0 / 300

1.021

$\mathrm{R} 1=0.0265, \mathrm{wR} 2=0.0493$

$\mathrm{R} 1=0.0369, \mathrm{wR} 2=0.0522$

1.879 and -1.399 e. $\AA^{-3}$

Table S4. Selected bond lengths $[\AA]$ and angles $\left[{ }^{\circ}\right]$ for $[\mathrm{U}($ crypt $)(\mathrm{MeCN}) \mathrm{I}][\mathrm{I}]_{2}$, 4-U.

\begin{tabular}{lrlr}
\hline $\mathrm{U}(1)-\mathrm{O}(4)$ & $2.597(2)$ & $\mathrm{U}(1)-\mathrm{O}(2)$ & $2.679(2)$ \\
$\mathrm{U}(1)-\mathrm{O}(5)$ & $2.598(2)$ & $\mathrm{U}(1)-\mathrm{N}(2)$ & $2.796(3)$ \\
$\mathrm{U}(1)-\mathrm{O}(1)$ & $2.627(2)$ & $\mathrm{U}(1)-\mathrm{N}(3)$ & $2.840(3)$ \\
$\mathrm{U}(1)-\mathrm{U}(1)$ & $2.647(3)$ & $\mathrm{U}(1)-\mathrm{I}(1)$ & $3.2594(3)$ \\
$\mathrm{U}(1)-\mathrm{O}(6)$ & $2.662(2)$ & & $1.139(4)$ \\
$\mathrm{U}(1)-\mathrm{O}(3)$ & $2.676(2)$ & & \\
& & & \\
$\mathrm{O}(4)-\mathrm{U}(1)-\mathrm{O}(5)$ & $103.22(7)$ & $\mathrm{O}(6)-\mathrm{U}(1)-\mathrm{O}(2)$ & $104.03(7)$ \\
$\mathrm{O}(4)-\mathrm{U}(1)-\mathrm{O}(1)$ & $148.63(7)$ & $\mathrm{O}(3)-\mathrm{U}(1)-\mathrm{O}(2)$ & $150.93(7)$ \\
$\mathrm{O}(5)-\mathrm{U}(1)-\mathrm{O}(1)$ & $102.20(7)$ & $\mathrm{O}(4)-\mathrm{U}(1)-\mathrm{N}(2)$ & $122.10(8)$ \\
$\mathrm{O}(4)-\mathrm{U}(1)-\mathrm{U}(1)$ & $137.45(8)$ & $\mathrm{O}(1)-\mathrm{U}(1)-\mathrm{N}(2)$ & $57.63(8)$ \\
$\mathrm{O}(5)-\mathrm{U}(1)-\mathrm{N}(1)$ & $68.22(8)$ & $\mathrm{N}(1)-\mathrm{U}(1)-\mathrm{N}(2)$ & $59.74(8)$ \\
$\mathrm{O}(1)-\mathrm{U}(1)-\mathrm{N}(1)$ & $69.99(8)$ & $\mathrm{O}(6)-\mathrm{U}(1)-\mathrm{N}(2)$ & $89.51(9)$ \\
$\mathrm{O}(4)-\mathrm{U}(1)-\mathrm{O}(6)$ & $73.53(7)$ & $\mathrm{O}(2)-\mathrm{U}(1)-\mathrm{N}(2)$ & $117.63(8)$ \\
$\mathrm{O}(5)-\mathrm{U}(1)-\mathrm{O}(6)$ & $60.03(7)$ & $\mathrm{O}(4)-\mathrm{U}(1)-\mathrm{N}(3)$ & $61.66(8)$ \\
$\mathrm{O}(1)-\mathrm{U}(1)-\mathrm{O}(6)$ & $136.19(7)$ & $\mathrm{O}(5)-\mathrm{U}(1)-\mathrm{N}(3)$ & $119.50(8)$ \\
$\mathrm{N}(1)-\mathrm{U}(1)-\mathrm{O}(6)$ & $66.22(8)$ & $\mathrm{O}(1)-\mathrm{U}(1)-\mathrm{N}(3)$ & $120.94(8)$ \\
$\mathrm{O}(4)-\mathrm{U}(1)-\mathrm{O}(3)$ & $60.46(7)$ & $\mathrm{N}(1)-\mathrm{U}(1)-\mathrm{N}(3)$ & $120.53(7)$ \\
$\mathrm{O}(5)-\mathrm{U}(1)-\mathrm{O}(3)$ & $70.63(7)$ & $\mathrm{O}(6)-\mathrm{U}(1)-\mathrm{N}(3)$ & $89.07(9)$ \\
$\mathrm{O}(1)-\mathrm{U}(1)-\mathrm{O}(3)$ & $112.42(7)$ & $\mathrm{O}(3)-\mathrm{U}(1)-\mathrm{N}(3)$ & $60.92(8)$ \\
$\mathrm{N}(1)-\mathrm{U}(1)-\mathrm{O}(3)$ & $138.14(8)$ & $\mathrm{O}(2)-\mathrm{U}(1)-\mathrm{N}(3)$ & $119.06(7)$ \\
$\mathrm{O}(6)-\mathrm{U}(1)-\mathrm{O}(3)$ & $99.28(7)$ & $\mathrm{N}(2)-\mathrm{U}(1)-\mathrm{N}(3)$ & $178.73(7)$ \\
$\mathrm{O}(4)-\mathrm{U}(1)-\mathrm{O}(2)$ & $110.10(7)$ & $\mathrm{O}(4)-\mathrm{U}(1)-\mathrm{I}(1)$ & $73.69(5)$ \\
$\mathrm{O}(5)-\mathrm{U}(1)-\mathrm{O}(2)$ & $136.81(8)$ & $59.79(7)$ & $141.15(5)$ \\
$\mathrm{O}(1)-\mathrm{U}(1)-\mathrm{O}(2)$ & $68.68(8)$ & & \\
$\mathrm{N}(1)-\mathrm{U}(1)-\mathrm{O}(2)$ & & &
\end{tabular}




$\begin{array}{lrll}\mathrm{O}(1)-\mathrm{U}(1)-\mathrm{I}(1) & 75.00(5) & \mathrm{C}(10)-\mathrm{O}(4)-\mathrm{U}(1) & 121.13(18) \\ \mathrm{N}(1)-\mathrm{U}(1)-\mathrm{I}(1) & 139.25(6) & \mathrm{C}(11)-\mathrm{O}(4)-\mathrm{U}(1) & 126.64(19) \\ \mathrm{O}(6)-\mathrm{U}(1)-\mathrm{I}(1) & 144.91(5) & \mathrm{C}(15)-\mathrm{O}(5)-\mathrm{U}(1) & 119.6(2) \\ \mathrm{O}(3)-\mathrm{U}(1)-\mathrm{I}(1) & 74.85(5) & \mathrm{C}(14)-\mathrm{O}(5)-\mathrm{U}(1) & 125.3(2) \\ \mathrm{O}(2)-\mathrm{U}(1)-\mathrm{I}(1) & 76.09(5) & \mathrm{C}(16)-\mathrm{O}(6)-\mathrm{U}(1) & 121.5(2) \\ \mathrm{N}(2)-\mathrm{U}(1)-\mathrm{I}(1) & 90.47(6) & \mathrm{C}(17)-\mathrm{O}(6)-\mathrm{U}(1) & 119.7(2) \\ \mathrm{N}(3)-\mathrm{U}(1)-\mathrm{I}(1) & 91.19(6) & \mathrm{C}(19)-\mathrm{N}(1)-\mathrm{U}(1) & 169.4(3) \\ \mathrm{C}(2)-\mathrm{O}(1)-\mathrm{U}(1) & 127.62(19) & \mathrm{C}(13)-\mathrm{N}(2)-\mathrm{U}(1) & 104.96(19) \\ \mathrm{C}(3)-\mathrm{O}(1)-\mathrm{U}(1) & 120.90(18) & \mathrm{C}(1)-\mathrm{N}(2)-\mathrm{U}(1) & 109.68(19) \\ \mathrm{C}(5)-\mathrm{O}(2)-\mathrm{U}(1) & 123.34(19) & \mathrm{C}(7)-\mathrm{N}(2)-\mathrm{U}(1) & 114.0(2) \\ \mathrm{C}(4)-\mathrm{O}(2)-\mathrm{U}(1) & 119.13(18) & \mathrm{C}(12)-\mathrm{N}(3)-\mathrm{U}(1) & 104.45(18) \\ \mathrm{C}(9)-\mathrm{O}(3)-\mathrm{U}(1) & 118.67(18) & \mathrm{C}(6)-\mathrm{N}(3)-\mathrm{U}(1) & 112.09(19) \\ \mathrm{C}(8)-\mathrm{O}(3)-\mathrm{U}(1) & 117.82(19) & \mathrm{C}(18)-\mathrm{N}(3)-\mathrm{U}(1) & 113.2(2)\end{array}$

X-ray Data Collection, Structure Solution and Refinement for $\left.\left[\mathrm{U}(\mathrm{crypt})\left(\mathrm{OH}_{2}\right)_{2}\right]_{[\mathrm{I}}\right]_{3} \cdot 2 \mathrm{MeCN}, \mathbf{5 - U}$.

A green crystal of approximate dimensions $0.169 \times 0.238 \times 0.289 \mathrm{~mm}$ was mounted in a cryoloop and transferred to a Bruker SMART APEX II diffractometer. The APEX $2{ }^{16}$ program package was used to determine the unit-cell parameters and for data collection $(10 \mathrm{sec} /$ frame scan time for a sphere of diffraction data). The raw frame data was processed using SAINT ${ }^{17}$ and SADABS ${ }^{18}$ to yield the reflection data file. Subsequent calculations were carried out using the SHELXTL ${ }^{19}$ program. The diffraction symmetry was $2 / m$ and the systematic absences were consistent with the monoclinic space group $P 2_{1} / n$ that was later determined to be correct.

The structure was solved by dual space methods and refined on $\mathrm{F}^{2}$ by full-matrix least-squares techniques. The analytical scattering factors ${ }^{20}$ for neutral atoms were used throughout the analysis. A disordered solvent molecule $(\mathrm{MeCN})$ was model in 2 parts (85:15). Hydrogen atoms were included using a riding model except for hydrogen atoms on coordinated water molecules were located from a difference-Fourier map and refined (x,y,z and $\left.\mathrm{U}_{\text {iso }}\right)$.

Least-squares analysis yielded $w R 2=0.494$ and Goof $=1.106$ for 347 variables refined against 8499 data $(0.74 \AA), \mathrm{R} 1=0.0231$ for those 8040 data with $\mathrm{I}>2.0 \sigma(\mathrm{I})$.

Table S5. Crystal data and structure refinement for $\left[\mathrm{U}(\mathrm{crypt})\left(\mathrm{OH}_{2}\right)_{2}\right][\mathrm{I}]_{3} \cdot 2 \mathrm{MeCN}, \mathbf{5 - U}$.

Identification code

Empirical formula

Formula weight

Temperature

Wavelength

Crystal system

Space group

Unit cell dimensions dnh88 (Dan Huh)

$\mathrm{C}_{22} \mathrm{H}_{46} \mathrm{I}_{3} \mathrm{~N}_{4} \mathrm{O}_{8} \mathrm{U}$

1113.36

133(2) K

$0.71073 \AA$

Monoclinic

$\mathrm{P} 2{ }_{1} / \mathrm{n}$

$\mathrm{a}=9.5283(10) \AA \quad \alpha=90^{\circ}$.

$\mathrm{b}=10.7944(12) \AA \quad \beta=96.2676(13)^{\circ}$. 
Volume

Z

Density (calculated)

Absorption coefficient

$\mathrm{F}(000)$

Crystal color

Crystal size

Theta range for data collection

Index ranges

Reflections collected

Independent reflections

Completeness to theta $=25.500^{\circ}$

Absorption correction

Max. and min. transmission

Refinement method

Data / restraints / parameters

Goodness-of-fit on $\mathrm{F}^{2}$

Final $\mathrm{R}$ indices $[\mathrm{I}>2 \operatorname{sigma}(\mathrm{I})=8040$ data]

$\mathrm{R}$ indices (all data, $0.74 \AA$ )

Largest diff. peak and hole $\mathrm{c}=33.824(4) \AA \quad \gamma=90^{\circ}$.

3458.1(6) $\AA^{3}$

4

$2.138 \mathrm{Mg} / \mathrm{m}^{3}$

$7.416 \mathrm{~mm}^{-1}$

2084

green

$0.289 \times 0.238 \times 0.169 \mathrm{~mm}^{3}$

1.211 to $28.832^{\circ}$.

$-12<=\mathrm{h}<=12,-14<=\mathrm{k}<=14,-45<=\mathrm{l}<=45$

40643

$8499[\mathrm{R}($ int $)=0.0405]$

$100.0 \%$

Semi-empirical from equivalents

0.4316 and 0.3407

Full-matrix least-squares on $\mathrm{F}^{2}$

8499 / 0 / 359

1.107

$\mathrm{R} 1=0.0231, \mathrm{wR} 2=0.0486$

$\mathrm{R} 1=0.0254, \mathrm{wR} 2=0.0493$

1.551 and -0.858 e. $\AA^{-3}$

Table S6. Selected bond lengths $[\AA ̊ \AA]$ and angles $\left[{ }^{\circ}\right]$ for $\left[\mathrm{U}(\mathrm{crypt})\left(\mathrm{OH}_{2}\right)_{2}\right][\mathrm{I}]_{3} \cdot 2 \mathrm{MeCN}, \mathbf{5}-\mathbf{U}$.

\begin{tabular}{lrlr}
\hline $\mathrm{U}(1)-\mathrm{O}(7)$ & $2.515(2)$ & $\mathrm{U}(1)-\mathrm{O}(2)$ & $2.631(2)$ \\
$\mathrm{U}(1)-\mathrm{O}(8)$ & $2.519(3)$ & $\mathrm{U}(1)-\mathrm{O}(1)$ & $2.633(2)$ \\
$\mathrm{U}(1)-\mathrm{O}(5)$ & $2.595(2)$ & $\mathrm{U}(1)-\mathrm{O}(4)$ & $2.677(2)$ \\
$\mathrm{U}(1)-\mathrm{O}(6)$ & $2.599(2)$ & $\mathrm{U}(1)-\mathrm{N}(2)$ & $2.746(3)$ \\
$\mathrm{U}(1)-\mathrm{O}(3)$ & $2.626(2)$ & $\mathrm{U}(1)-\mathrm{N}(1)$ & $2.816(3)$ \\
& & & \\
$\mathrm{O}(7)-\mathrm{U}(1)-\mathrm{O}(8)$ & $134.77(9)$ & $\mathrm{O}(5)-\mathrm{U}(1)-\mathrm{O}(6)$ & $62.51(7)$ \\
$\mathrm{O}(7)-\mathrm{U}(1)-\mathrm{O}(5)$ & $66.08(8)$ & $\mathrm{O}(7)-\mathrm{U}(1)-\mathrm{O}(3)$ & $133.91(8)$ \\
$\mathrm{O}(8)-\mathrm{U}(1)-\mathrm{O}(5)$ & $141.08(8)$ & $\mathrm{O}(8)-\mathrm{U}(1)-\mathrm{O}(3)$ & $86.14(8)$ \\
$\mathrm{O}(7)-\mathrm{U}(1)-\mathrm{O}(6)$ & $74.68(8)$ & $\mathrm{O}(5)-\mathrm{U}(1)-\mathrm{O}(3)$ & $68.06(7)$ \\
$\mathrm{O}(8)-\mathrm{U}(1)-\mathrm{O}(6)$ & $143.45(8)$ & $\mathrm{O}(6)-\mathrm{U}(1)-\mathrm{O}(3)$ & $80.70(7)$
\end{tabular}




\begin{tabular}{|c|c|c|c|}
\hline $\mathrm{O}(7)-\mathrm{U}(1)-\mathrm{O}(2)$ & $66.10(8)$ & $\mathrm{O}(8)-\mathrm{U}(1)-\mathrm{N}(1)$ & $80.05(8)$ \\
\hline $\mathrm{O}(8)-\mathrm{U}(1)-\mathrm{O}(2)$ & $75.14(8)$ & $\mathrm{O}(5)-\mathrm{U}(1)-\mathrm{N}(1)$ & $62.16(8)$ \\
\hline $\mathrm{O}(5)-\mathrm{U}(1)-\mathrm{O}(2)$ & $131.67(7)$ & $\mathrm{O}(6)-\mathrm{U}(1)-\mathrm{N}(1)$ & $120.77(7)$ \\
\hline $\mathrm{O}(6)-\mathrm{U}(1)-\mathrm{O}(2)$ & $110.24(7)$ & $\mathrm{O}(3)-\mathrm{U}(1)-\mathrm{N}(1)$ & $60.54(8)$ \\
\hline $\mathrm{O}(3)-\mathrm{U}(1)-\mathrm{O}(2)$ & $159.97(7)$ & $\mathrm{O}(2)-\mathrm{U}(1)-\mathrm{N}(1)$ & $121.43(7)$ \\
\hline $\mathrm{O}(7)-\mathrm{U}(1)-\mathrm{O}(1)$ & $76.03(8)$ & $\mathrm{O}(1)-\mathrm{U}(1)-\mathrm{N}(1)$ & $61.31(7)$ \\
\hline $\mathrm{O}(8)-\mathrm{U}(1)-\mathrm{O}(1)$ & $64.65(8)$ & $\mathrm{O}(4)-\mathrm{U}(1)-\mathrm{N}(1)$ & $111.42(7)$ \\
\hline $\mathrm{O}(5)-\mathrm{U}(1)-\mathrm{O}(1)$ & $101.53(7)$ & $\mathrm{N}(2)-\mathrm{U}(1)-\mathrm{N}(1)$ & $169.31(8)$ \\
\hline $\mathrm{O}(6)-\mathrm{U}(1)-\mathrm{O}(1)$ & $150.43(7)$ & $\mathrm{C}(1)-\mathrm{N}(1)-\mathrm{U}(1)$ & $109.07(19)$ \\
\hline $\mathrm{O}(3)-\mathrm{U}(1)-\mathrm{O}(1)$ & $118.05(7)$ & $\mathrm{C}(13)-\mathrm{N}(1)-\mathrm{U}(1)$ & $104.45(18)$ \\
\hline $\mathrm{O}(2)-\mathrm{U}(1)-\mathrm{O}(1)$ & $60.13(7)$ & $\mathrm{C}(7)-\mathrm{N}(1)-\mathrm{U}(1)$ & $114.55(19)$ \\
\hline $\mathrm{O}(7)-\mathrm{U}(1)-\mathrm{O}(4)$ & $145.46(8)$ & $\mathrm{C}(6)-\mathrm{N}(2)-\mathrm{U}(1)$ & $109.65(19)$ \\
\hline $\mathrm{O}(8)-\mathrm{U}(1)-\mathrm{O}(4)$ & $66.10(8)$ & $\mathrm{C}(12)-\mathrm{N}(2)-\mathrm{U}(1)$ & $105.55(19)$ \\
\hline $\mathrm{O}(5)-\mathrm{U}(1)-\mathrm{O}(4)$ & $117.84(7)$ & $\mathrm{C}(18)-\mathrm{N}(2)-\mathrm{U}(1)$ & $111.69(19)$ \\
\hline $\mathrm{O}(6)-\mathrm{U}(1)-\mathrm{O}(4)$ & $77.89(7)$ & $\mathrm{C}(2)-\mathrm{O}(1)-\mathrm{U}(1)$ & $125.81(18)$ \\
\hline $\mathrm{O}(3)-\mathrm{U}(1)-\mathrm{O}(4)$ & $59.43(7)$ & $\mathrm{C}(3)-\mathrm{O}(1)-\mathrm{U}(1)$ & $120.31(18)$ \\
\hline $\mathrm{O}(2)-\mathrm{U}(1)-\mathrm{O}(4)$ & $105.34(7)$ & $\mathrm{C}(5)-\mathrm{O}(2)-\mathrm{U}(1)$ & $124.72(18)$ \\
\hline $\mathrm{O}(1)-\mathrm{U}(1)-\mathrm{O}(4)$ & $130.72(7)$ & $\mathrm{C}(4)-\mathrm{O}(2)-\mathrm{U}(1)$ & $122.33(18)$ \\
\hline $\mathrm{O}(7)-\mathrm{U}(1)-\mathrm{N}(2)$ & $90.00(9)$ & $\mathrm{C}(9)-\mathrm{O}(3)-\mathrm{U}(1)$ & $116.52(19)$ \\
\hline $\mathrm{O}(8)-\mathrm{U}(1)-\mathrm{N}(2)$ & $91.39(8)$ & $\mathrm{C}(8)-\mathrm{O}(3)-\mathrm{U}(1)$ & $112.79(18)$ \\
\hline $\mathrm{O}(5)-\mathrm{U}(1)-\mathrm{N}(2)$ & $124.83(8)$ & $\mathrm{C}(10)-\mathrm{O}(4)-\mathrm{U}(1)$ & $124.25(19)$ \\
\hline $\mathrm{O}(6)-\mathrm{U}(1)-\mathrm{N}(2)$ & $63.44(7)$ & $\mathrm{C}(11)-\mathrm{O}(4)-\mathrm{U}(1)$ & $122.87(19)$ \\
\hline $\mathrm{O}(3)-\mathrm{U}(1)-\mathrm{N}(2)$ & $112.86(8)$ & $\mathrm{C}(14)-\mathrm{O}(5)-\mathrm{U}(1)$ & $124.30(19)$ \\
\hline $\mathrm{O}(2)-\mathrm{U}(1)-\mathrm{N}(2)$ & $61.49(7)$ & $\mathrm{C}(15)-\mathrm{O}(5)-\mathrm{U}(1)$ & $119.16(19)$ \\
\hline $\mathrm{O}(1)-\mathrm{U}(1)-\mathrm{N}(2)$ & $120.67(8)$ & $\mathrm{C}(17)-\mathrm{O}(6)-\mathrm{C}(16)$ & $109.1(2)$ \\
\hline $\mathrm{O}(4)-\mathrm{U}(1)-\mathrm{N}(2)$ & $58.75(8)$ & $\mathrm{C}(17)-\mathrm{O}(6)-\mathrm{U}(1)$ & $119.91(18)$ \\
\hline $\mathrm{O}(7)-\mathrm{U}(1)-\mathrm{N}(1)$ & $100.56(8)$ & $\mathrm{C}(16)-\mathrm{O}(6)-\mathrm{U}(1)$ & $118.03(19)$ \\
\hline
\end{tabular}


Table S7. Hydrogen bonds for $\left[\mathrm{U}(\mathrm{crypt})\left(\mathrm{OH}_{2}\right)_{2}\right][\mathrm{I}]_{3} \cdot 2 \mathrm{MeCN}, \mathbf{5}-\mathbf{U}$, [A and $\left.{ }^{\circ}\right]$.

\begin{tabular}{lllll}
\hline D-H...A & d(D-H) & d(H...A) & $\mathrm{d}(\mathrm{D} \ldots \mathrm{A})$ & $<(\mathrm{DHA})$ \\
\hline $\mathrm{O}(8)-\mathrm{H}(8 \mathrm{~B}) \ldots \mathrm{I}(1)$ & $0.70(5)$ & $2.91(5)$ & $3.576(3)$ & $163(5)$ \\
O(7)-H(7A)..I(3)\#1 & $0.84(5)$ & $2.67(5)$ & $3.501(3)$ & $168(4)$ \\
O(8)-H(8A)...I(1)\#2 & $0.81(5)$ & $2.63(5)$ & $3.413(3)$ & $162(4)$ \\
O(7)-H(7B)..I(2)\#3 & $0.79(5)$ & $2.77(5)$ & $3.546(3)$ & $170(5)$
\end{tabular}

Symmetry transformations used to generate equivalent atoms:

$\# 1 \mathrm{x}-1, \mathrm{y}, \mathrm{z} \quad \# 2-\mathrm{x}+1,-\mathrm{y},-\mathrm{z} \quad \# 3-\mathrm{x}+1 / 2, \mathrm{y}-1 / 2,-\mathrm{z}+1 / 2$

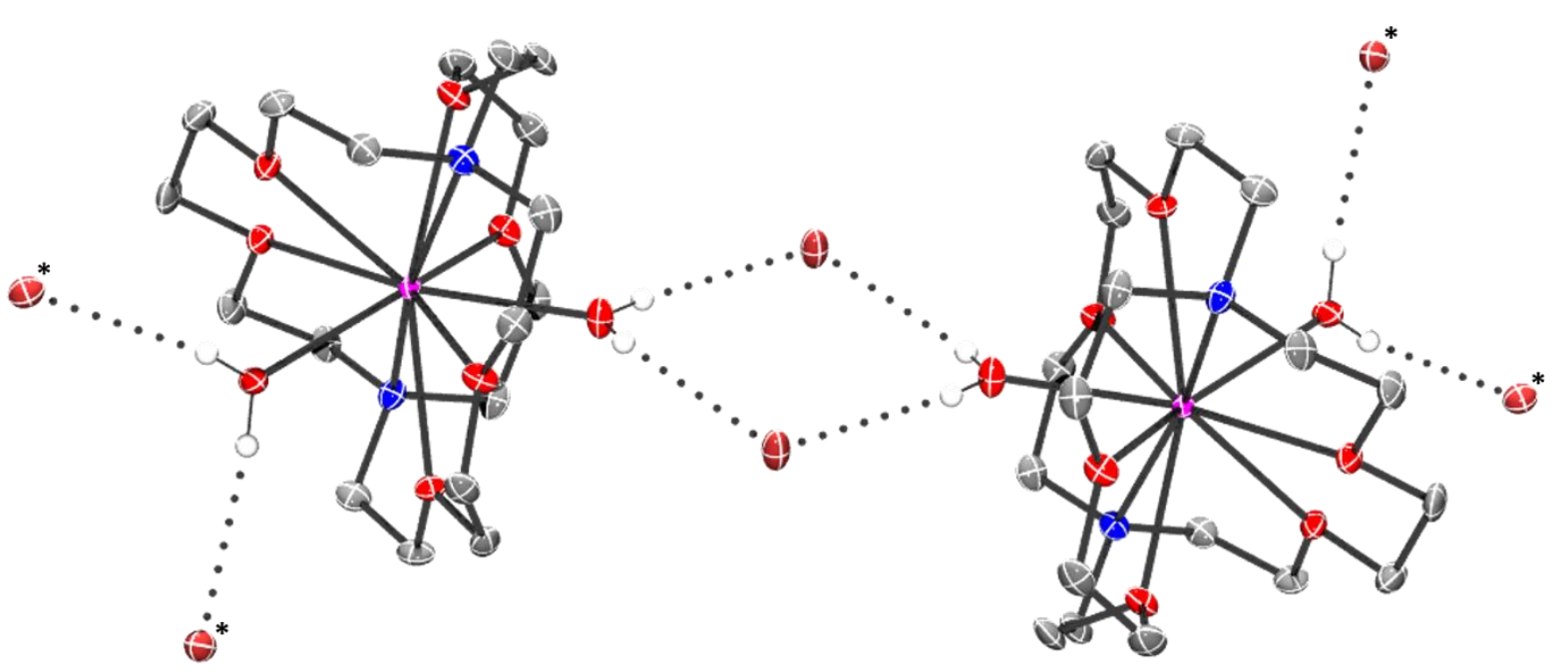

Figure S28. Hydrogen bonding between two units of $\left[\mathrm{U}(\mathrm{crypt})\left(\mathrm{OH}_{2}\right)_{2}\right][\mathrm{I}]_{3}, \mathbf{5}-\mathbf{U}$. Outer-sphere $\mathrm{MeCN}$ and hydrogen atoms are omitted except $\mathrm{OH}_{2}$ hydrogens. Iodides marked with an asterisk $(*)$ do not bridge to a unit of $\left[\mathrm{U}(\operatorname{crypt})\left(\mathrm{OH}_{2}\right)_{2}\right]^{3+}$. Magenta $(\mathrm{U})$, red $(\mathrm{O})$, blue $(\mathrm{N})$, brown $(\mathrm{I})$, white $(\mathrm{H})$.

X-ray Data Collection, Structure Solution and Refinement for $\left[\mathrm{U}(\mathrm{crypt})(\mathrm{DMF})\left(\mathrm{OH}_{2}\right)\right][\mathrm{I}]_{3} \cdot \mathrm{H}_{2} \mathrm{O}$, 6-U.

A green crystal of approximate dimensions $0.044 \times 0.063 \times 0.069 \mathrm{~mm}$ was mounted in a cryoloop and transferred to a Bruker SMART APEX II diffractometer. The APEX $2{ }^{16}$ program package was used to determine the unit-cell parameters and for data collection (240 sec/frame scan time for a sphere of diffraction data). The raw frame data was processed using SAINT ${ }^{17}$ and SADABS $^{18}$ to yield the reflection data file. Subsequent calculations were carried out using the SHELXTL ${ }^{19}$ program. The diffraction symmetry was $2 / m$ and the systematic absences were consistent with the monoclinic space group $P 2{ }_{1} / n$ that was later determined to be correct. 
The structure was solved by direct methods and refined on $\mathrm{F}^{2}$ by full-matrix least-squares techniques. The analytical scattering factors ${ }^{20}$ for neutral atoms were used throughout the analysis. Hydrogen atoms were included using a riding model. Carbon atom $\mathrm{C}(14)$ was disordered and modeled using multiple components and with partial site-occupancy-factors (0.25:0.75). There was one molecule of free water present which was hydrogen bonded to both the bound water $\mathrm{O}(8)$ and iodine atoms $\mathrm{I}(1)$ and $\mathrm{I}(2)$.

Least-squares analysis yielded $\mathrm{wR} 2=0.0736$ and Goof $=0.992$ for 339 variables refined against 6825 data $(0.80 \AA), \mathrm{R} 1=0.0432$ for those 4915 data with $\mathrm{I}>2.0 \sigma(\mathrm{I})$.

Table S8. Crystal data and structure refinement for $\left[\mathrm{U}(\mathrm{crypt})(\mathrm{DMF})\left(\mathrm{OH}_{2}\right)\right][\mathrm{I}]_{3} \cdot \mathrm{H}_{2} \mathrm{O}, \mathbf{6}-\mathbf{U}$.

Identification code

Empirical formula

Formula weight

Temperature

Wavelength

Crystal system

Space group

Unit cell dimensions

Volume

$\mathrm{Z}$

Density (calculated)

Absorption coefficient

$\mathrm{F}(000)$

Crystal color

Crystal size

Theta range for data collection

Index ranges

Reflections collected

Independent reflections

Completeness to theta $=25.500^{\circ}$

Absorption correction

Max. and min. transmission

Refinement method dnh78 (Dan Huh)

$\mathrm{C}_{21} \mathrm{H}_{47} \mathrm{I}_{3} \mathrm{~N}_{3} \mathrm{O}_{\underline{9}} \mathrm{U}$

1104.34

88(2) K

$0.71073 \AA$

Monoclinic

$P 2_{1} / n$

$a=17.7598(15) \AA$

$\alpha=90^{\circ}$.

$\mathrm{b}=11.2951(10) \AA$

$\beta=111.1464(10)^{\circ}$.

$c=17.8077(15) \AA$

$\gamma=90^{\circ}$.

3331.7(5) $\AA^{3}$

4

$2.202 \mathrm{Mg} / \mathrm{m}^{3}$

$7.697 \mathrm{~mm}^{-1}$

2068

green

$0.069 \times 0.063 \times 0.044 \mathrm{~mm}^{3}$

1.388 to $26.397^{\circ}$

$-22 \leq h \leq 22,-14 \leq k \leq 14,-22 \leq l \leq 22$

35688

6825 [R(int) $=0.0999]$

$100.0 \%$

Semi-empirical from equivalents

0.4296 and 0.3683

Full-matrix least-squares on $\mathrm{F}^{2}$ 
Data / restraints / parameters

Goodness-of-fit on $\mathrm{F}^{2}$

Final $\mathrm{R}$ indices $[\mathrm{I}>2 \operatorname{sigma}(\mathrm{I})=4915$ data]

$\mathrm{R}$ indices (all data, $0.80 \AA$ )

Largest diff. peak and hole
6825 / 0 / 339

0.992

$\mathrm{R} 1=0.0432, \mathrm{wR} 2=0.0651$

$\mathrm{R} 1=0.0780, \mathrm{wR} 2=0.0736$

1.260 and -1.032 e. $\AA^{-3}$

Table S9. Selected bond lengths $\left[\AA \AA^{\circ}\right]$ and angles $\left[{ }^{\circ}\right]$ for $\left[\mathrm{U}(\operatorname{crypt})(\mathrm{DMF})\left(\mathrm{OH}_{2}\right)\right][\mathrm{I}]{ }^{\cdot} \cdot \mathrm{H}_{2} \mathrm{O}, \mathbf{6}-\mathbf{U}$.

\begin{tabular}{|c|c|c|c|}
\hline $\mathrm{U}(1)-\mathrm{O}(7)$ & $2.416(5)$ & $\mathrm{U}(1)-\mathrm{O}(4)$ & $2.652(5)$ \\
\hline $\mathrm{U}(1)-\mathrm{O}(8)$ & $2.518(4)$ & $\mathrm{U}(1)-\mathrm{O}(5)$ & $2.656(5)$ \\
\hline $\mathrm{U}(1)-\mathrm{O}(3)$ & $2.592(5)$ & $\mathrm{U}(1)-\mathrm{N}(1)$ & $2.756(6)$ \\
\hline $\mathrm{U}(1)-\mathrm{O}(1)$ & $2.594(5)$ & $\mathrm{U}(1)-\mathrm{N}(2)$ & $2.769(5)$ \\
\hline $\mathrm{U}(1)-\mathrm{O}(2)$ & $2.602(4)$ & $\mathrm{U}(1)-\mathrm{O}(6)$ & $2.778(5)$ \\
\hline $\mathrm{O}(7)-\mathrm{U}(1)-\mathrm{O}(8)$ & $136.77(16)$ & $\mathrm{O}(3)-\mathrm{U}(1)-\mathrm{N}(2)$ & $122.52(16)$ \\
\hline $\mathrm{O}(7)-\mathrm{U}(1)-\mathrm{O}(3)$ & $72.95(16)$ & $\mathrm{O}(1)-\mathrm{U}(1)-\mathrm{N}(2)$ & $125.89(16)$ \\
\hline $\mathrm{O}(8)-\mathrm{U}(1)-\mathrm{O}(3)$ & $73.77(15)$ & $\mathrm{O}(2)-\mathrm{U}(1)-\mathrm{N}(2)$ & $64.08(16)$ \\
\hline $\mathrm{O}(7)-\mathrm{U}(1)-\mathrm{O}(1)$ & $142.41(16)$ & $\mathrm{O}(4)-\mathrm{U}(1)-\mathrm{N}(2)$ & $62.25(15)$ \\
\hline $\mathrm{O}(8)-\mathrm{U}(1)-\mathrm{O}(1)$ & $67.79(15)$ & $\mathrm{O}(5)-\mathrm{U}(1)-\mathrm{N}(2)$ & $110.91(16)$ \\
\hline $\mathrm{O}(3)-\mathrm{U}(1)-\mathrm{O}(1)$ & $95.99(16)$ & $\mathrm{N}(1)-\mathrm{U}(1)-\mathrm{N}(2)$ & $167.79(17)$ \\
\hline $\mathrm{O}(7)-\mathrm{U}(1)-\mathrm{O}(2)$ & $142.56(15)$ & $\mathrm{O}(7)-\mathrm{U}(1)-\mathrm{O}(6)$ & $69.32(16)$ \\
\hline $\mathrm{O}(8)-\mathrm{U}(1)-\mathrm{O}(2)$ & $70.03(14)$ & $\mathrm{O}(8)-\mathrm{U}(1)-\mathrm{O}(6)$ & $137.60(15)$ \\
\hline $\mathrm{O}(3)-\mathrm{U}(1)-\mathrm{O}(2)$ & $142.78(14)$ & $\mathrm{O}(3)-\mathrm{U}(1)-\mathrm{O}(6)$ & $142.27(15)$ \\
\hline $\mathrm{O}(1)-\mathrm{U}(1)-\mathrm{O}(2)$ & $62.51(15)$ & $\mathrm{O}(1)-\mathrm{U}(1)-\mathrm{O}(6)$ & $114.01(16)$ \\
\hline $\mathrm{O}(7)-\mathrm{U}(1)-\mathrm{O}(4)$ & $76.36(16)$ & $\mathrm{O}(2)-\mathrm{U}(1)-\mathrm{O}(6)$ & $74.24(14)$ \\
\hline $\mathrm{O}(8)-\mathrm{U}(1)-\mathrm{O}(4)$ & $63.52(15)$ & $\mathrm{O}(4)-\mathrm{U}(1)-\mathrm{O}(6)$ & $109.06(15)$ \\
\hline $\mathrm{O}(3)-\mathrm{U}(1)-\mathrm{O}(4)$ & $60.62(15)$ & $\mathrm{O}(5)-\mathrm{U}(1)-\mathrm{O}(6)$ & $56.24(15)$ \\
\hline $\mathrm{O}(1)-\mathrm{U}(1)-\mathrm{O}(4)$ & $130.04(15)$ & $\mathrm{N}(1)-\mathrm{U}(1)-\mathrm{O}(6)$ & $112.82(16)$ \\
\hline $\mathrm{O}(2)-\mathrm{U}(1)-\mathrm{O}(4)$ & $108.54(14)$ & $\mathrm{N}(2)-\mathrm{U}(1)-\mathrm{O}(6)$ & $56.91(16)$ \\
\hline $\mathrm{O}(7)-\mathrm{U}(1)-\mathrm{O}(5)$ & $83.68(17)$ & $\mathrm{C}(3)-\mathrm{O}(1)-\mathrm{U}(1)$ & $117.3(4)$ \\
\hline $\mathrm{O}(8)-\mathrm{U}(1)-\mathrm{O}(5)$ & $137.57(16)$ & $\mathrm{C}(2)-\mathrm{O}(1)-\mathrm{U}(1)$ & $123.8(4)$ \\
\hline $\mathrm{O}(3)-\mathrm{U}(1)-\mathrm{O}(5)$ & $119.90(15)$ & $\mathrm{C}(4)-\mathrm{O}(2)-\mathrm{U}(1)$ & $118.8(4)$ \\
\hline $\mathrm{O}(1)-\mathrm{U}(1)-\mathrm{O}(5)$ & $70.78(16)$ & $\mathrm{C}(5)-\mathrm{O}(2)-\mathrm{U}(1)$ & $116.9(4)$ \\
\hline $\mathrm{O}(2)-\mathrm{U}(1)-\mathrm{O}(5)$ & $83.28(15)$ & $\mathrm{C}(8)-\mathrm{O}(3)-\mathrm{U}(1)$ & $126.8(4)$ \\
\hline $\mathrm{O}(4)-\mathrm{U}(1)-\mathrm{O}(5)$ & $158.87(16)$ & $\mathrm{C}(9)-\mathrm{O}(3)-\mathrm{U}(1)$ & $119.5(4)$ \\
\hline $\mathrm{O}(7)-\mathrm{U}(1)-\mathrm{N}(1)$ & $81.15(17)$ & $\mathrm{C}(11)-\mathrm{O}(4)-\mathrm{U}(1)$ & $122.9(4)$ \\
\hline $\mathrm{O}(8)-\mathrm{U}(1)-\mathrm{N}(1)$ & $105.16(16)$ & $\mathrm{C}(10)-\mathrm{O}(4)-\mathrm{U}(1)$ & $120.3(4)$ \\
\hline $\mathrm{O}(3)-\mathrm{U}(1)-\mathrm{N}(1)$ & $60.39(16)$ & $\mathrm{C}(14 \mathrm{~A})-\mathrm{O}(5)-\mathrm{C}(15)$ & $122.1(13)$ \\
\hline $\mathrm{O}(1)-\mathrm{U}(1)-\mathrm{N}(1)$ & $62.72(16)$ & $\mathrm{C}(14 \mathrm{~B})-\mathrm{O}(5)-\mathrm{C}(15)$ & 113.1(6) \\
\hline $\mathrm{O}(2)-\mathrm{U}(1)-\mathrm{N}(1)$ & $121.90(16)$ & $\mathrm{C}(14 \mathrm{~A})-\mathrm{O}(5)-\mathrm{U}(1)$ & $113.7(13)$ \\
\hline $\mathrm{O}(4)-\mathrm{U}(1)-\mathrm{N}(1)$ & $120.63(16)$ & $\mathrm{C}(14 \mathrm{~B})-\mathrm{O}(5)-\mathrm{U}(1)$ & $122.9(5)$ \\
\hline $\mathrm{O}(5)-\mathrm{U}(1)-\mathrm{N}(1)$ & $61.78(16)$ & $\mathrm{C}(15)-\mathrm{O}(5)-\mathrm{U}(1)$ & $118.9(4)$ \\
\hline $\mathrm{O}(7)-\mathrm{U}(1)-\mathrm{N}(2)$ & $88.45(17)$ & $\mathrm{C}(16)-\mathrm{O}(6)-\mathrm{U}(1)$ & $124.7(5)$ \\
\hline $\mathrm{O}(8)-\mathrm{U}(1)-\mathrm{N}(2)$ & $86.80(16)$ & $\mathrm{C}(17)-\mathrm{O}(6)-\mathrm{U}(1)$ & $123.5(4)$ \\
\hline
\end{tabular}


$\mathrm{C}(19)-\mathrm{O}(7)-\mathrm{U}(1)$

$\mathrm{C}(7)-\mathrm{N}(1)-\mathrm{U}(1)$

$\mathrm{C}(13)-\mathrm{N}(1)-\mathrm{U}(1)$

$\mathrm{C}(1)-\mathrm{N}(1)-\mathrm{U}(1)$
$140.5(5)$

$108.4(4)$

$115.1(4)$

105.2(4)
$\mathrm{C}(6)-\mathrm{N}(2)-\mathrm{U}(1)$

$\mathrm{C}(18)-\mathrm{N}(2)-\mathrm{U}(1)$

$\mathrm{C}(12)-\mathrm{N}(2)-\mathrm{U}(1)$
$110.4(4)$

$107.7(4)$

$110.7(4)$

Table S10. Hydrogen bonds for $\left[\mathrm{U}(\mathrm{crypt})(\mathrm{DMF})\left(\mathrm{OH}_{2}\right)\right]\left[[]_{3} \cdot \mathrm{H}_{2} \mathrm{O}, \mathbf{6}-\mathbf{U},\left[\AA\right.\right.$ and $\left.^{\circ}\right]$.

\begin{tabular}{lcccc}
\hline D-H...A & d(D-H) & d(H...A) & d(D...A) & $<($ DHA $)$ \\
\hline $\mathrm{O}(8)-\mathrm{H}(8 \mathrm{~A}) \ldots \mathrm{O}(9) \# 1$ & 0.87 & 2.10 & $2.812(7)$ & 138.4 \\
$\mathrm{O}(8)-\mathrm{H}(8 \mathrm{~B}) \ldots \mathrm{O}(9)$ & 0.87 & 2.31 & $2.773(7)$ & 113.5 \\
$\mathrm{O}(9)-\mathrm{H}(9 \mathrm{C}) \ldots \mathrm{I}(1)$ & 0.87 & 2.65 & $3.517(5)$ & 172.9 \\
$\mathrm{O}(9)-\mathrm{H}(9 \mathrm{D}) \ldots \mathrm{I}(2)$ & 0.87 & 2.55 & $3.405(5)$ & 167.1
\end{tabular}

Symmetry transformations used to generate equivalent atoms:

$\# 1-\mathrm{x}+1,-\mathrm{y}+1,-\mathrm{z}+1$
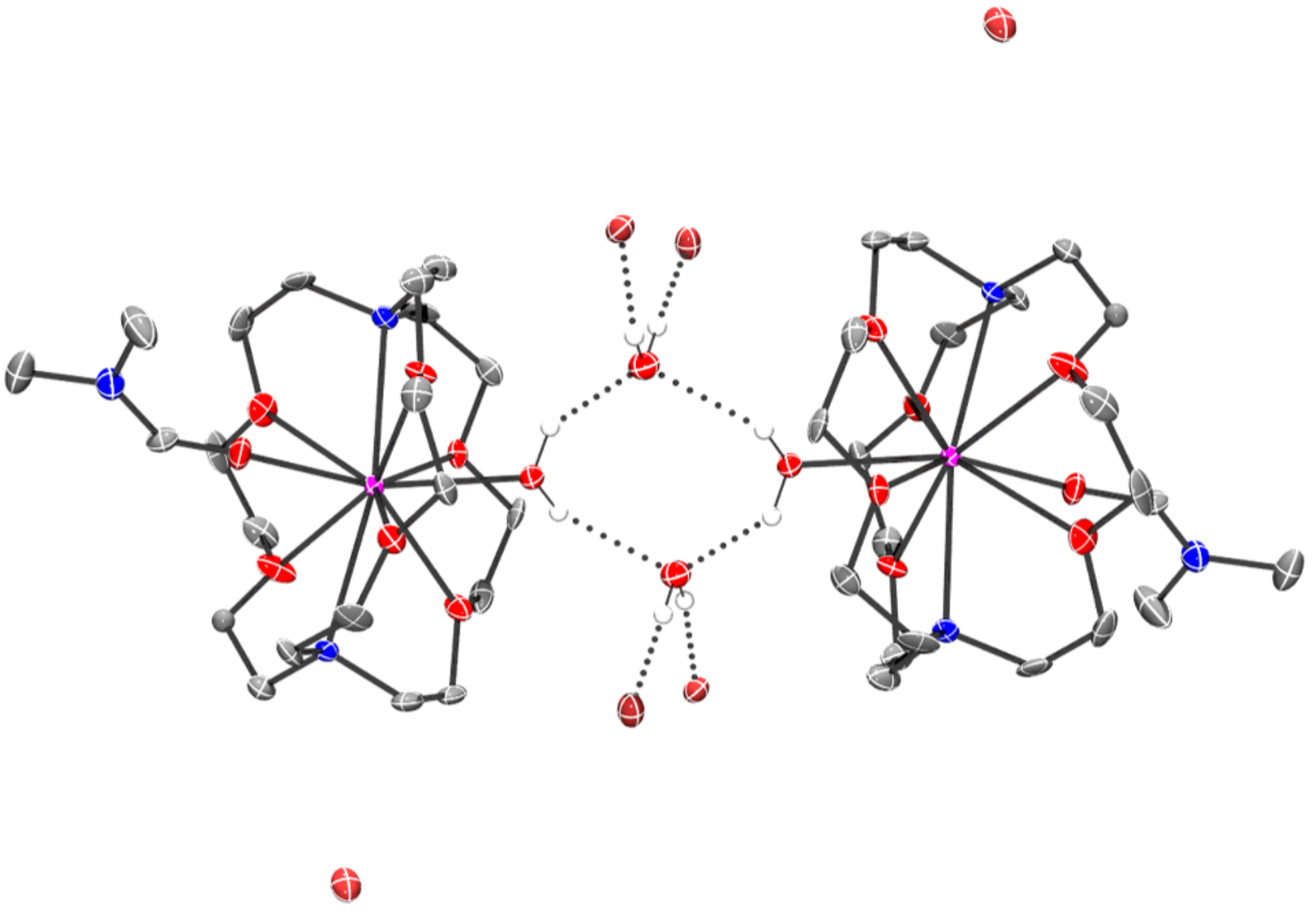

Figure S29. Hydrogen bonding between two units of $\left[\mathrm{U}(\mathrm{crypt})(\mathrm{DMF})\left(\mathrm{OH}_{2}\right)\right][\mathrm{I}]_{3}, \mathbf{6}-\mathrm{U}$. Hydrogen atoms are omitted except $\mathrm{OH}_{2}$ hydrogens. Magenta $(\mathrm{U})$, red $(\mathrm{O})$, blue $(\mathrm{N})$, brown $(\mathrm{I})$, white $(\mathrm{H})$. 
X-ray Data Collection, Structure Solution and Refinement for $\left[\mathrm{H}_{2}\right.$ (crypt) $][\mathrm{I}]_{2}$.

A colorless crystal of approximate dimensions $0.304 \times 0.145 \times 0.105 \mathrm{~mm}$ was mounted in a cryoloop and transferred to a Bruker SMART APEX II diffractometer. The APEX2 $2^{16}$ program package was used to determine the unit-cell parameters and for data collection $(15 \mathrm{sec} /$ frame scan time for a sphere of diffraction data). The raw frame data was processed using SAINT $^{17}$ and SADABS $^{18}$ to yield the reflection data file. Subsequent calculations were carried out using the SHELXTL ${ }^{19}$ program. The diffraction symmetry was $\mathrm{mmm}$ and the systematic absences were consistent with the orthorhombic space group $P b c n$ that was later determined to be correct.

The structure was solved by dual space methods and refined on $\mathrm{F}^{2}$ by full-matrix least-squares techniques. The analytical scattering factor ${ }^{20}$ for neutral atoms were used throughout the analysis. Hydrogen atoms were included using a riding model and the $\mathrm{N}-\mathrm{H}$ hydrogen was located from a difference-Fourier map and refined (x,y,z and $\mathrm{U}_{\text {iso }}$ ). Hydrogen bonding between $\mathrm{N}-\mathrm{H} . . \mathrm{O} 1, \mathrm{~N}-$ $\mathrm{H} . . . \mathrm{O} 2$, and N-H...O3' has been identified. Disordered carbons were modeled isotropically in two parts 75:25.

Least-squares analysis yielded $\mathrm{wR} 2=0.0621$ and Goof $=1.046$ for 126 variables refined against 3405 data $(0.72 \AA), \mathrm{R} 1=0.0286$ for those 2825 data with $\mathrm{I}>2.0 \sigma(\mathrm{I})$.

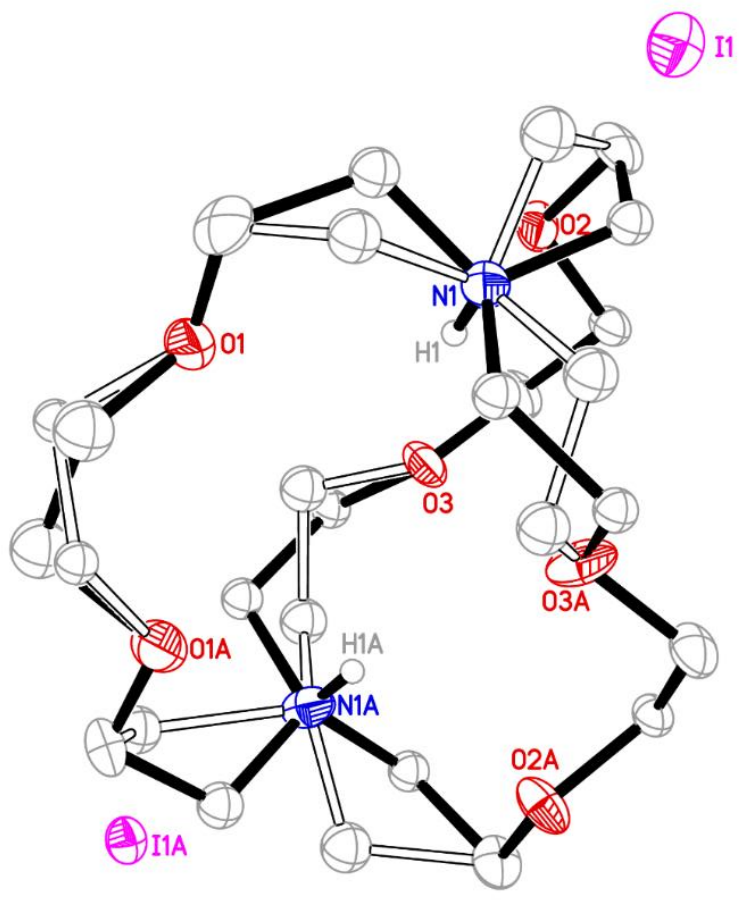

Figure S30. Solid state structure of $\left[\mathrm{H}_{2}(\mathrm{crypt})\right][\mathrm{I}]_{2}$ formed upon oxidation of $[\mathrm{U}(\mathrm{crypt}) \mathrm{I}][\mathrm{I}]_{2}$ with 1 eq. of $\left[\mathrm{Fe}\left(\mathrm{C}_{5} \mathrm{H}_{5}\right)_{2}\right]\left[\mathrm{BF}_{4}\right]$. 
Table S11. Crystal data and structure refinement for $\left[\mathrm{H}_{2}(\mathrm{crypt})\right][\mathrm{I}]_{2}$.

Identification code

Empirical formula

Formula weight

Temperature

Wavelength

Crystal system

Space group

Unit cell dimensions

Volume

Z

Density (calculated)

Absorption coefficient

$\mathrm{F}(000)$

Crystal color

Crystal size

Theta range for data collection

Index ranges

Reflections collected

Independent reflections

Completeness to theta $=25.500^{\circ}$

Absorption correction

Max. and min. transmission

Refinement method

Data / restraints / parameters

Goodness-of-fit on $\mathrm{F}^{2}$

Final $\mathrm{R}$ indices $[\mathrm{I}>2 \operatorname{sigma}(\mathrm{I})=2825$ data]

$\mathrm{R}$ indices (all data, $0.72 \AA$ )

Largest diff. peak and hole jyy 189

$\mathrm{C}_{18} \mathrm{H}_{37} \mathrm{I}_{2} \mathrm{~N}_{2} \mathrm{O}_{6}$

631.29

93(2) K

$0.71073 \AA$

Orthorhombic

$\mathrm{Pbcn}$

$\mathrm{a}=19.7599(15) \AA \quad \alpha=90^{\circ}$.

$\mathrm{b}=9.6890(7) \AA \quad \beta=90^{\circ}$.

$\mathrm{c}=12.6449(9) \AA \quad \gamma=90^{\circ}$.

2420.9(3) $\AA^{3}$

4

$1.732 \mathrm{Mg} / \mathrm{m}^{3}$

$2.631 \mathrm{~mm}^{-1}$

1252

colorless

$0.304 \times 0.145 \times 0.105 \mathrm{~mm}^{3}$

2.061 to $29.573^{\circ}$.

$-27<=\mathrm{h}<=27,-13<=\mathrm{k}<=13,-17<=\mathrm{l}<=17$

34340

$3405[\mathrm{R}(\mathrm{int})=0.0339]$

$100.0 \%$

Semi-empirical from equivalents

0.5650 and 0.4479

Full-matrix least-squares on $\mathrm{F}^{2}$

3405 / 0 / 126

1.046

$\mathrm{R} 1=0.0286, \mathrm{wR} 2=0.0571$

$\mathrm{R} 1=0.0386, \mathrm{wR} 2=0.0621$

1.158 and -0.952 e. $\AA^{-3}$ 
Table S12. Bond lengths $[\AA]$ and angles $\left[^{\circ}\right]$ for $\left[\mathrm{H}_{2}(\right.$ crypt $\left.)\right][]_{2}$.

\begin{tabular}{llll}
\hline $\mathrm{O}(1)-\mathrm{C}(2)$ & $1.418(4)$ & $\mathrm{N}(1)-\mathrm{C}(1 \mathrm{~B})$ & $1.753(11)$ \\
$\mathrm{O}(1)-\mathrm{C}(3 \mathrm{~B})$ & $1.455(10)$ & $\mathrm{C}(1 \mathrm{~A})-\mathrm{C}(2)$ & $1.502(5)$ \\
$\mathrm{O}(1)-\mathrm{C}(3 \mathrm{~A})$ & $1.462(5)$ & $\mathrm{C}(2)-\mathrm{C}(1 \mathrm{~B})$ & $1.366(11)$ \\
$\mathrm{O}(2)-\mathrm{C}(5)$ & $1.421(3)$ & $\mathrm{C}(3 \mathrm{~A})-\mathrm{C}(3 \mathrm{~A}) \# 1$ & $1.491(8)$ \\
$\mathrm{O}(2)-\mathrm{C}(6)$ & $1.422(3)$ & $\mathrm{C}(4 \mathrm{~A})-\mathrm{C}(5)$ & $1.476(4)$ \\
$\mathrm{O}(3)-\mathrm{C}(9 \mathrm{~B}) \# 1$ & $1.355(11)$ & $\mathrm{C}(5)-\mathrm{C}(4 \mathrm{~B})$ & $1.566(12)$ \\
$\mathrm{O}(3)-\mathrm{C}(7)$ & $1.413(3)$ & $\mathrm{C}(6)-\mathrm{C}(7)$ & $1.500(4)$ \\
$\mathrm{O}(3)-\mathrm{C}(9 \mathrm{~A}) \# 1$ & $1.472(4)$ & $\mathrm{C}(8 \mathrm{~A})-\mathrm{C}(9 \mathrm{~A})$ & $1.511(5)$ \\
$\mathrm{N}(1)-\mathrm{C}(1 \mathrm{~A})$ & $1.465(4)$ & $\mathrm{C}(9 \mathrm{~A})-\mathrm{O}(3) \# 1$ & $1.472(4)$ \\
$\mathrm{N}(1)-\mathrm{C}(8 \mathrm{~A})$ & $1.483(4)$ & $\mathrm{C}(3 \mathrm{~B})-\mathrm{C}(3 \mathrm{~B}) \# 1$ & $1.502(19)$ \\
$\mathrm{N}(1)-\mathrm{C}(4 \mathrm{~A})$ & $1.522(4)$ & $\mathrm{C}(8 \mathrm{~B})-\mathrm{C}(9 \mathrm{~B})$ & $1.458(16)$ \\
$\mathrm{N}(1)-\mathrm{C}(4 \mathrm{~B})$ & $1.541(12)$ & $\mathrm{C}(9 \mathrm{~B})-\mathrm{O}(3) \# 1$ & $1.355(11)$ \\
$\mathrm{N}(1)-\mathrm{C}(8 \mathrm{~B})$ & $1.544(11)$ & & \\
& & & \\
$\mathrm{C}(2)-\mathrm{O}(1)-\mathrm{C}(3 \mathrm{~B})$ & $126.6(4)$ & $\mathrm{O}(1)-\mathrm{C}(3 \mathrm{~A})-\mathrm{C}(3 \mathrm{~A}) \# 1$ & $108.0(3)$ \\
$\mathrm{C}(2)-\mathrm{O}(1)-\mathrm{C}(3 \mathrm{~A})$ & $104.1(3)$ & $\mathrm{C}(5)-\mathrm{C}(4 \mathrm{~A})-\mathrm{N}(1)$ & $112.4(3)$ \\
$\mathrm{C}(5)-\mathrm{O}(2)-\mathrm{C}(6)$ & $113.2(2)$ & $\mathrm{O}(2)-\mathrm{C}(5)-\mathrm{C}(4 \mathrm{~A})$ & $109.9(2)$ \\
$\mathrm{C}(9 \mathrm{~B}) \# 1-\mathrm{O}(3)-\mathrm{C}(7)$ & $126.8(5)$ & $\mathrm{O}(2)-\mathrm{C}(5)-\mathrm{C}(4 \mathrm{~B})$ & $112.5(5)$ \\
$\mathrm{C}(7)-\mathrm{O}(3)-\mathrm{C}(9 \mathrm{~A}) \# 1$ & $107.5(2)$ & $\mathrm{O}(2)-\mathrm{C}(6)-\mathrm{C}(7)$ & $107.4(2)$ \\
$\mathrm{C}(1 \mathrm{~A})-\mathrm{N}(1)-\mathrm{C}(8 \mathrm{~A})$ & $115.5(3)$ & $\mathrm{O}(3)-\mathrm{C}(7)-\mathrm{C}(6)$ & $109.6(2)$ \\
$\mathrm{C}(1 \mathrm{~A})-\mathrm{N}(1)-\mathrm{C}(4 \mathrm{~A})$ & $112.1(2)$ & $\mathrm{N}(1)-\mathrm{C}(8 \mathrm{~A})-\mathrm{C}(9 \mathrm{~A})$ & $114.2(3)$ \\
$\mathrm{C}(8 \mathrm{~A})-\mathrm{N}(1)-\mathrm{C}(4 \mathrm{~A})$ & $113.3(2)$ & $\mathrm{O}(3) \# 1-\mathrm{C}(9 \mathrm{~A})-\mathrm{C}(8 \mathrm{~A})$ & $105.1(3)$ \\
$\mathrm{C}(4 \mathrm{~B})-\mathrm{N}(1)-\mathrm{C}(8 \mathrm{~B})$ & $110.7(6)$ & $\mathrm{O}(1)-\mathrm{C}(3 \mathrm{~B})-\mathrm{C}(3 \mathrm{~B}) \# 1$ & $105.7(7)$ \\
$\mathrm{C}(4 \mathrm{~B})-\mathrm{N}(1)-\mathrm{C}(1 \mathrm{~B})$ & $98.8(6)$ & $\mathrm{N}(1)-\mathrm{C}(4 \mathrm{~B})-\mathrm{C}(5)$ & $106.7(7)$ \\
$\mathrm{C}(8 \mathrm{~B})-\mathrm{N}(1)-\mathrm{C}(1 \mathrm{~B})$ & $97.0(6)$ & $\mathrm{C}(2)-\mathrm{C}(1 \mathrm{~B})-\mathrm{N}(1)$ & $106.2(7)$ \\
$\mathrm{N}(1)-\mathrm{C}(1 \mathrm{~A})-\mathrm{C}(2)$ & $115.2(3)$ & $\mathrm{C}(9 \mathrm{~B})-\mathrm{C}(8 \mathrm{~B})-\mathrm{N}(1)$ & $106.6(9)$ \\
$\mathrm{C}(1 \mathrm{~B})-\mathrm{C}(2)-\mathrm{O}(1)$ & $117.2(5)$ & $\mathrm{O}(3) \# 1-\mathrm{C}(9 \mathrm{~B})-\mathrm{C}(8 \mathrm{~B})$ & $105.6(9)$ \\
$\mathrm{O}(1)-\mathrm{C}(2)-\mathrm{C}(1 \mathrm{~A})$ & $109.7(2)$ & &
\end{tabular}

Table S13. Hydrogen bonds for $\left[\mathrm{H}_{2}\right.$ (crypt) $][\mathrm{I}]_{2}\left[\AA\right.$ and $\left.^{\circ}\right]$.

\begin{tabular}{lcccc}
\hline $\mathrm{D}-\mathrm{H} \ldots \mathrm{A}$ & $\mathrm{d}(\mathrm{D}-\mathrm{H})$ & $\mathrm{d}(\mathrm{H} \ldots \mathrm{A})$ & $\mathrm{d}(\mathrm{D} \ldots \mathrm{A})$ & $<(\mathrm{DHA})$ \\
\hline $\mathrm{N}(1)-\mathrm{H}(1) \ldots \mathrm{O}(1)$ & $0.89(4)$ & $2.52(4)$ & $2.831(3)$ & $101(3)$ \\
$\mathrm{N}(1)-\mathrm{H}(1) \ldots \mathrm{O}(2)$ & $0.89(4)$ & $2.33(4)$ & $2.742(3)$ & $108(3)$ \\
$\mathrm{N}(1)-\mathrm{H}(1) \ldots \mathrm{O}(3) \# 1$ & $0.89(4)$ & $2.49(4)$ & $2.799(3)$ & $101(3)$ \\
\hline
\end{tabular}

Symmetry transformations used to generate equivalent atoms:

$\# 1-\mathrm{x}+2, \mathrm{y},-\mathrm{z}+1 / 2$ 


\section{References}

1. Huh, D. N.; Windorff, C. J.; Ziller, J. W.; Evans, W. J., Synthesis of uranium-in-cryptand complexes. Chemical Communications 2018, 54 (73), 10272-10275.

2. Connelly, N. G.; Geiger, W. E., Chemical Redox Agents for Organometallic Chemistry. Chemical Reviews 1996, 96 (2), 877-910.

3. Hauchard, D.; Cassir, M.; Chivot, J.; Ephritikhine, M., Electrochemical study of uranium(IV) and uranium(IV) organometallic compounds in tetrahydrofuran by means of conventional microelectrodes and ultramicroelectrodes: Part I. Application to the $\mathrm{Na}(\mathrm{Hg})$ reduction of $\mathrm{Cp} 3 \mathrm{UCl}(\mathrm{Cp}=\eta-\mathrm{C} 5 \mathrm{H} 5)$. Journal of Electroanalytical Chemistry and Interfacial Electrochemistry 1991, 313 (1), 227-241.

4. Sonnenberger, D. C.; Gaudiello, J. G., Cyclic voltammetric study of organoactinide compounds of uranium(IV) and neptunium(IV). Ligand effects on the M(IV)/M(III) couple. Inorganic Chemistry 1988, 27 (15), 2747-2748.

5. $\quad$ Boreen, M. A.; Lussier, D. J.; Skeel, B. A.; Lohrey, T. D.; Watt, F. A.; Shuh, D. K.; Long, J. R.; Hohloch, S.; Arnold, J., Structural, Electrochemical, and Magnetic Studies of Bulky Uranium(III) and Uranium(IV) Metallocenes. Inorganic Chemistry 2019, 58 (24), 16629-16641. 6. Afonso, M. L.; Gomes, A.; Carvalho, A.; Alves, L. C.; Wastin, F.; Gonçalves, A. P., Electrochemical behaviour of uranium (IV) in DMF at vitreous carbon. Electrochimica Acta 2009, 54 (28), 7318-7323.

7. Cannes, C.; Le Naour, C.; Moisy, P.; Guilbaud, P., Specific Interaction between Uranium Anionic Complexes and the Cations of Bis(trifluoromethylsulfonyl)imide Based Ionic Liquids. Inorganic Chemistry 2013, 52 (19), 11218-11227.

8. Vallat, A.; Laviron, E.; Dormond, A., A comparative electrochemical study of thorium(IV) and uranium(IV) acetylacetonates. Journal of the Chemical Society, Dalton Transactions 1990, (3), 921-924.

9. Cantat, T.; Scott, B. L.; Morris, D. E.; Kiplinger, J. L., What a Difference a $5 f$ Element Makes: Trivalent and Tetravalent Uranium Halide Complexes Supported by One and Two Bis[2(diisopropylphosphino)-4-methylphenyl]amido (PNP) Ligands. Inorganic Chemistry 2009, 48 (5), 2114-2127.

10. Biswas, S.; Ma, S.; Nuzzo, S.; Twamley, B.; Russell, A. T.; Platts, J. A.; Hartl, F.; Baker, R. J., Structural Variability of $4 \mathrm{f}$ and $5 \mathrm{f}$ Thiocyanate Complexes and Dissociation of Uranium(III)-Thiocyanate Bonds with Increased Ionicity. Inorganic Chemistry 2017, 56 (23), 14426-14437.

11. Kim, S.-Y.; Harada, M.; Tomiyasu, H.; Shiokawa, Y.; Ikeda, Y., Electrochemical Study on Octakis(dimethyl sulfoxide)uranium(IV) Complex in Dimethyl Sulfoxide. Journal of Nuclear Science and Technology 2000, 37 (11), 999-1002.

12. Camp, C.; Andrez, J.; Pécaut, J.; Mazzanti, M., Synthesis of Electron-Rich Uranium(IV) Complexes Supported by Tridentate Schiff Base Ligands and Their Multi-Electron Redox Chemistry. Inorganic Chemistry 2013, 52 (12), 7078-7086.

13. Hümmer, J.; Heinemann, F. W.; Meyer, K., Uranium Tetrakis-Aryloxide Derivatives Supported by Tetraazacyclododecane: Synthesis of Air-Stable, Coordinatively-Unsaturated U(IV) and U(V) Complexes. Inorganic Chemistry 2017, 56 (6), 3201-3206.

14. Morris, D. E.; Da Re, R. E.; Jantunen, K. C.; Castro-Rodriguez, I.; Kiplinger, J. L., Trends in Electronic Structure and Redox Energetics for Early-Actinide Pentamethylcyclopentadienyl Complexes. Organometallics 2004, 23 (22), 5142-5153. 
15. Schelter, E. J.; Yang, P.; Scott, B. L.; Thompson, J. D.; Martin, R. L.; Hay, P. J.; Morris, D. E.; Kiplinger, J. L., Systematic Studies of Early Actinide Complexes: Uranium(IV)

Fluoroketimides. Inorganic Chemistry 2007, 46 (18), 7477-7488.

16. APEX2 Version 2014.11-0, Bruker AXS, Inc.; Madison, WI 2014.

17. SAINT Version 8.34a, Bruker AXS, Inc.; Madison, WI 2013.

18. Sheldrick, G. M. SADABS, Version 2014/5, Bruker AXS, Inc.; Madison, WI 2014.

19. Sheldrick, G. M. SHELXTL, Version 2014/7, Bruker AXS, Inc.; Madison, WI 2014

20. International Tables for Crystallography 1992, Vol. C., Dordrecht: Kluwer Academic Publishers. 